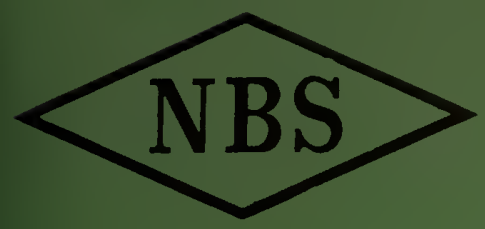

Reference book not to ka taken frnm ine library. $\quad$ PB 151377-3

\title{
NBS
}

Eechnical Note
Boulder Laboratories

QUARTERLY RADIO NOISE DATA -

JUNE, JULY, AUGUST 1959

BY W. Q. CRICHLOW, R.D. DISNEY, AND M. A. JENKINS

U. S. DEPARTMENT OF COMMERCE NATIONAL BUREAU OF STANDARDS

no. $18-3$ 


\section{THE NATIONAL BUREAU OF STANDARDS}

\section{Functions and Activities}

The functions of the National Bureau of Standards are set forth in the Act of Congress, March 3, 1901, as amended by Congress in Public Law 619, 1950. These include the development and maintenance of the national standards of measurement and the provision of means and methods for making measurements consistent with these standards: the determination of physical constants and properties of materials; the development of methods and instruments for testing materials, devires. and structures; advisory services to government agencies on scientific and technical problems; invention and development of devices to serve special needs of the Government; and the development of standard prartices, codes, and specifications. The work includes basic and applied rcscarch. development, engineering, instrumentation, testing, evaluation, calibration services, and various consultation and information services. Research projects are also performed for other government agencies when the work relates to and supplements the basic program of the Bureau or when the Bureau's unique competence is required. The scope of activities is suggested by the listing of divisions and sections on the inside of the back cover.

\section{Publications}

The results of the Bureau's work take the form of either actual equipment and devices or published papers. These papers appear either in the Bureau's own series of publications or in the journals of professional and scientific societies. The Bureau itself publishes three periodicals available froin the Government Printing Office: The Journal of Research, published in four separatc sections. presents complete scientific and technical papers; the Technical News Bulletin presents summary and preliminary reports on work in progress; and Basic Radio Propagation Predictions proviles data for determining the best frequencies to use for radio communications throughout the world. There are also five series of nonperiodical publications: Monographs, Applied Mathematics Scries, Handbooks, Misccllaneous Publications, and Technical Notes.

Information on the Bureau's publications can be found in NBS Circular 460, Publications of the Vational Bureau of Standards (\$1.25) and its Supplement (\$1.50), available from the Superintendent of Documents, Government Printing Office, Washington 25, D.C. 


\section{NATIONAL BUREAU OF STANDARDS}

\section{Eechnical Mote}

No. $18-3$

September 9, 1960

QUARTERLY RADIO NOISE DATA - JUNE, JULY, AUGUST 1959

by

W. Q. Crichlow, R. T. Disney, and M. A. Jenkins

NBS Technical Notes are designed to supplement the Bureau's regular publications program. They provide a means for making available scientific data that are of iransient or limited interest. Technical Notes may be listed or referred to in the open literature. They are for sale by the Office of Technical Services, U. S. Department of Commerce, Washington $25, \mathrm{D}$. C.

\section{DISTRIBUTED BY \\ UNITED STATES DEPARTMENT OF COMMERCE \\ OPFICE OF TECHNICAL SERVICES \\ WASHINGTON 25, D. C.}

Price $\$ 1.00$ 



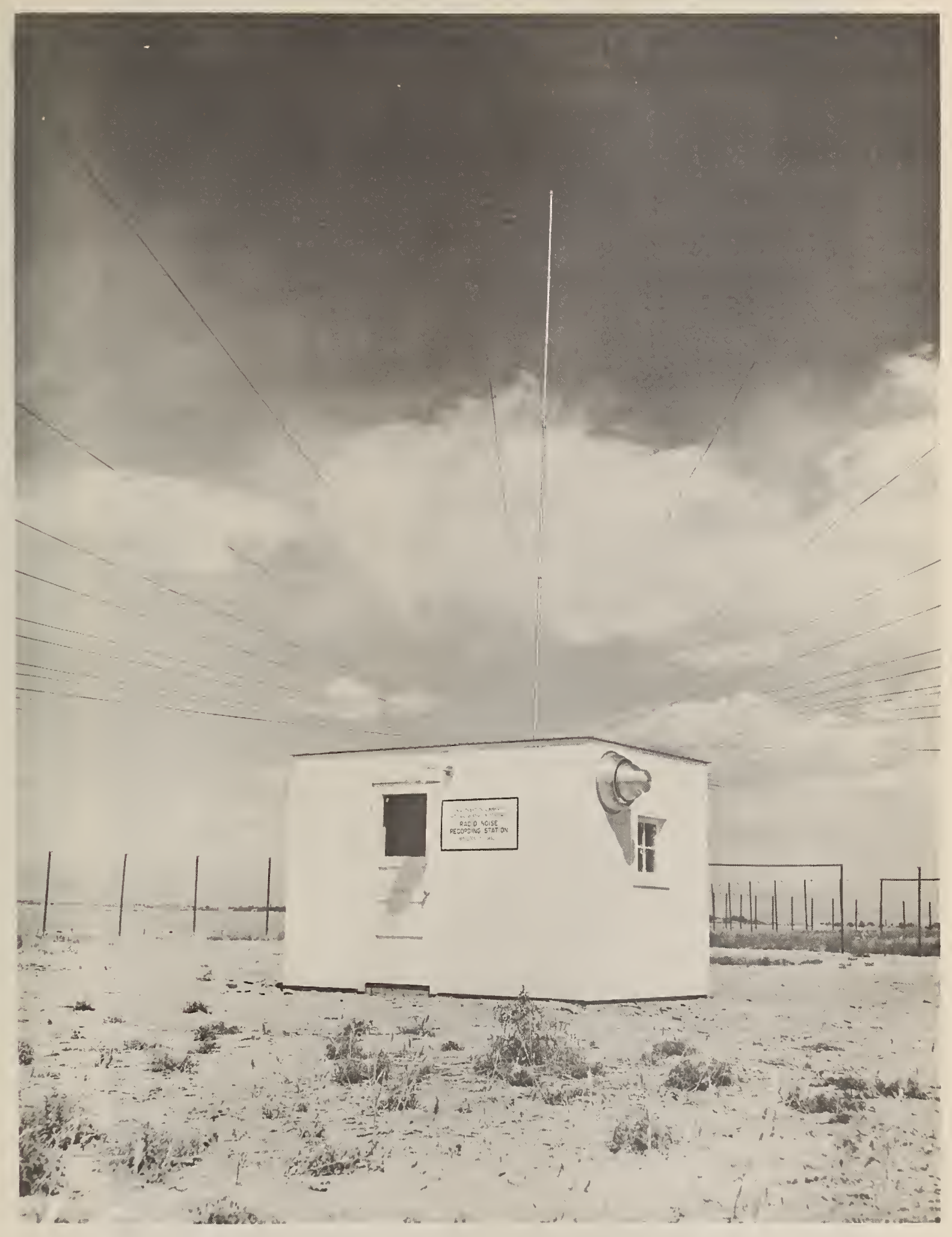

Radio Noise Recording Station 


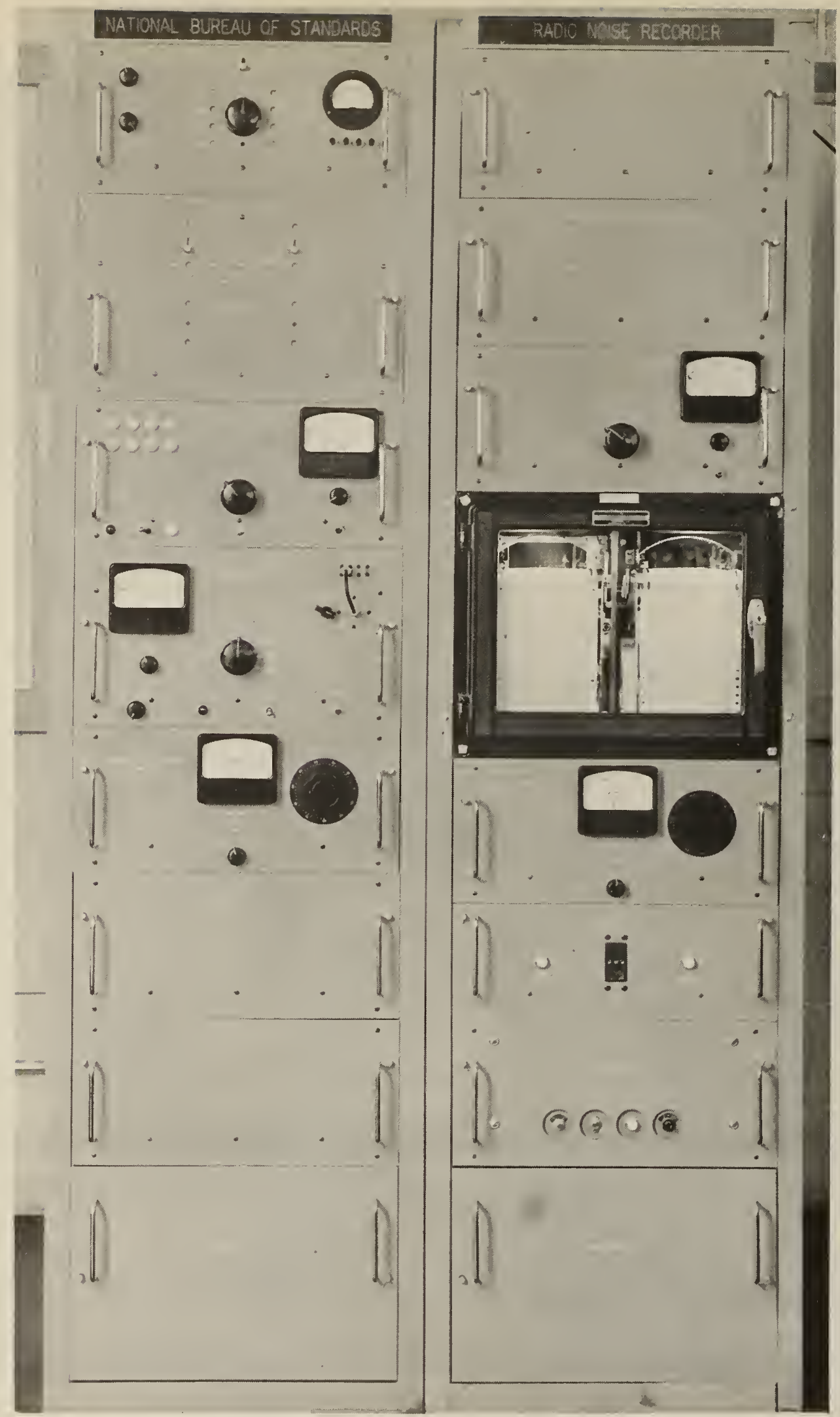

ARN-2 Atmospheric Radio Noise Recorder 


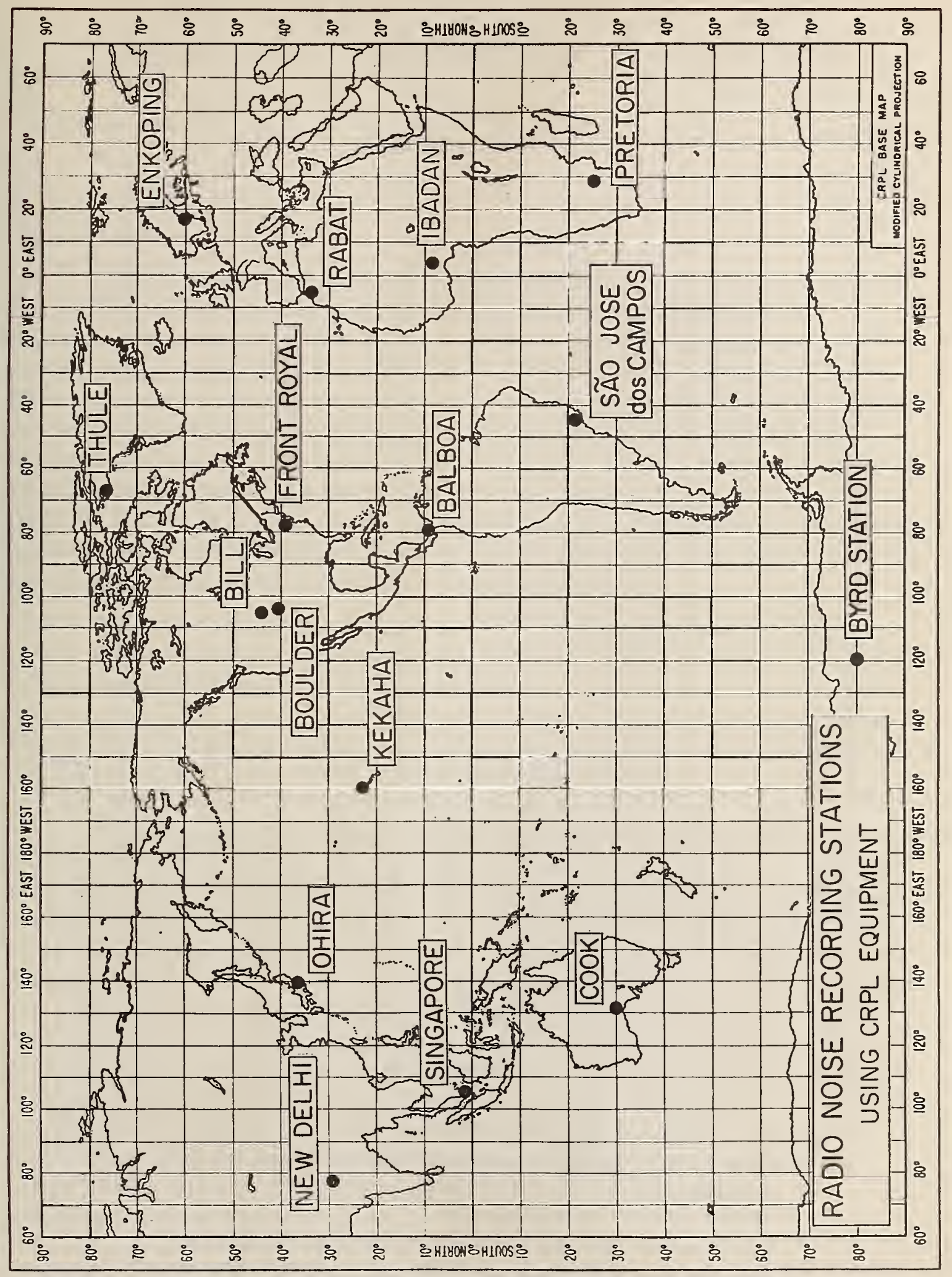


NOMOGRAM FOR TRANSFORMING EFFECTIVE ANTENNA NOISE FIGURE TO NOISE FIELD STRENGTH AS A FUNCTION OF FREQUENCY

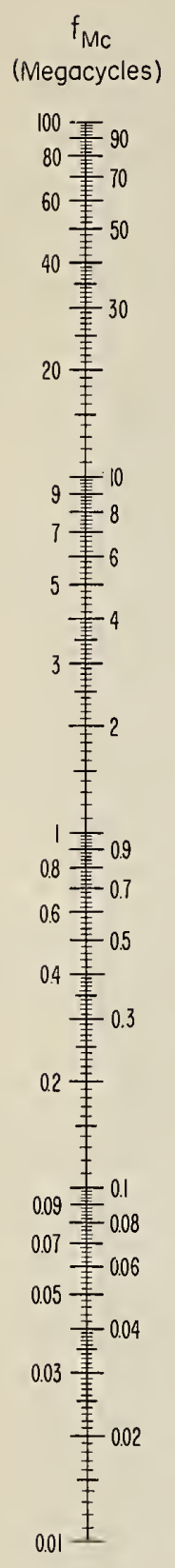

$\mathrm{Fa}_{\mathrm{a}}$

(db abave ktb)

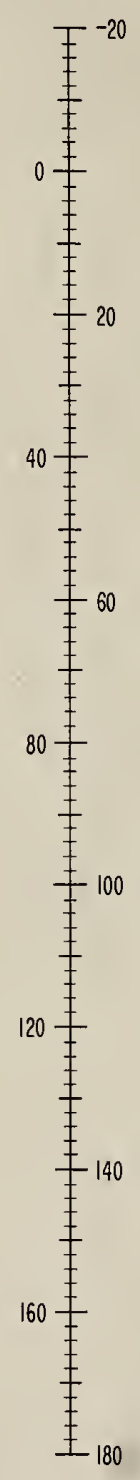

$E_{n}$

(db abave $1 \mu \mathrm{v} /$ meter)

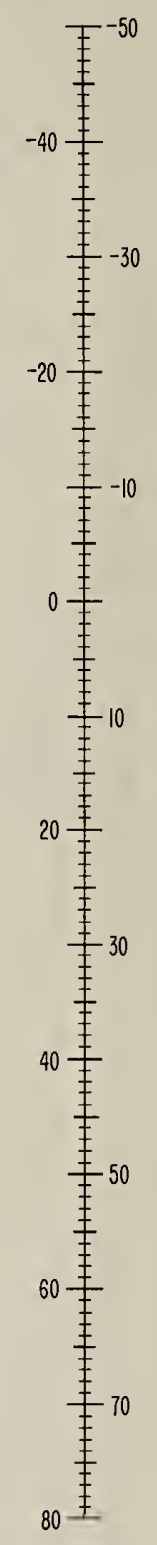

$$
E_{n}=F_{a}+20 \log _{10} f_{M c}-65.5
$$

$F_{a}=$ Effective Antenna Noise Figure $=$ External Noise Power Available from an Equivalent Short, Lossless, Vertical Antenna in $\mathrm{db}$ Above ktb.

$E_{n}=$ Equivalent Vertically Polarized Ground Wave R.M.S. Noise Field Strength in $\mathrm{db}$ Above $\mathrm{I} \mu \mathrm{v} /$ meter for a I kc Bandwidth.

$\mathrm{f}_{\mathrm{Mc}}=$ Frequency in Megacycles. 
Radio Noise Data for the Season June, July, August 1959

Radio noise measurements are being made at sixteen stations in a world-wide network supervised by the National Bureau of Standards (see map). The results of these measurements for the period June, July, August 1959 are presented in the attached tables. These are based on three parameters of the noise: (1) the mean power, (2) the mean envelope voltage, and (3) the mean logarithm of the envelope voltage. The mean power averaged over a perlod of several minutes is the basic parameter and is expressed as an effective antenna noise figure, $F_{a} . F_{a}$ is defined as the noise power available from an equivalent lossless antenna in $\mathrm{db}$ above $k$ tb (the thermal noise power available from a passive resistance) where

$k=$ Boltzman's constant ( $1.38 \times 10^{-23}$ joules per degree Kelvin)

$t=$ Absolute room temperature (taken as $288^{\circ} \mathrm{K}$ )

$b=$ Bandwidth in cycles per second.

The mean voltage and mean logarithm are expressed as deviations, $V_{d}$ and $I_{d}$, respectively, in $d b$ below the mean power.

Measurements of these parameters were made with the National Bureau of Standards Radio Noise Recorder, Model ARN-2, which has an effective noise bandwidth of about 200 cycles per second and uses a standard $21.75^{\prime}$ vertical antenna. A fifteen-minute recording is made on each of eight frequencies two at a time during each hour, and these fifteen-minute samples are taken as representing the noise conditions for the full hour. The month-hour medians, $F_{a m}, V_{d m}$ and $L_{d m}$ are determined from these hourly values for each of the corresponding parameters. Normally from twenty-five to thirty observations of the mean power are obtained monthly for each hour of the day, and from ten to fifteen observations of the voltage and logarithm deviations. When there are fewer than fifteen observations of the mean power, or seven observations of the voltage and logarithm deviations, the tabulated values are identified by an asterisk. 
The upper and lower decile values of $F_{a}$ are also reported in the following tabulation to give an indication of the extent of the variation of the noise power from day to day at a given time of day. These are expressed in $\mathrm{db}$ above and below the month-hour median, $F_{a m}$, and designated by $D_{u}$ and $D_{l}$, respectively.

Time-block median values of noise are tabulated on a seasonal basis, and are obtained by averaging all month-hour medians for the season within a particular four-hour period of the day. The timeblock values conform to the seasonal-time-block values used in C.C.I.R. Report No. 65 (see attached references).

$F_{a}$ in $d b$ is related to the rms fleld strength at the antenna by the following equation:

$$
E_{n}=F_{a}+20 \log _{10} f_{M c}-65.5
$$

where

$E_{n}=$ the equivalent vertically polarized ground wave rms noise field strength in $\mathrm{db}$ above $1 \mu \mathrm{v} /$ meter for a $1 \mathrm{kc}$ bandwidth. $f_{M c}=$ the frequency in megacycles/second.

The nomogram given may be used for this conversion.

The values presented in the tables reflect the actual measured radio noise; in some instances the atmospheric noise level may be contaminated by man-made noise or station interference. The parameter that will first reflect any such contamination will be the logarithmic parameter, $L_{d}$. This contamination generally will cause the value of $L_{d}$ to be less than it would have been, had the recorded value been only atmospheric noise. In determining the amplitudeprobability distribution from the three measured moments [10], contaminated values of $L_{d}$ may be found that will not give a solution of the amplitude-probability distribution. When this occurs, it is suggested that the measured value of $L_{d}$ be ignored and the most probable value of $L_{d}$ from the curve on the graph of $L_{d} v s . ~ V d$ be used. The most probable value has been determined as the best fit for the integrated moments from over slxty measured amplitude-probability distributions of uncontaminated atmospheric radio noise. The second curve on the graph indicates the minimum value of $L_{d}$ that will give an amplitude-probability distribution by the method in reference 10 , and 
can therefore be used to determine whether the measured value or the most probable value of $L_{d}$ for any value of $V_{d}$ should be used.

Station clocks are set to a local standard time (LST) which is taken from the time zone in which the station is located and is always an integral number of hours different than universal or Greenwich time (s ee table on page 5).

These preliminary data values are presented in order to expedite dissemination of the data. Additional analyses, in which an attempt is made to eliminate contaminated data, are presented in other publications.

Stations in the recording network were operated by the following agencies:

NBS - Bill, Wyoming; Boulder, Colorado; Byrd Station; Front Royal, Virginia; Kekaha, Hawail

Signal Corps, U. S. Army - Balboa, C. Z.; Thule, Greenland Postmaster General's Department (Australia) - Cook

Board of Telecommunications (Sweden) - Enkoping

DSIR (Great Britain) and University College Department of Physics (Nigeria) - Ibadan

Ministry of Communications, Wireless Planning and Co-ordination Organisation - New Delhi

Radio Research Laboratories (Japan) - Ohira.

Telecommunications Research Laboratory (South Africa) Pretoria

Institut Scientifique Chérifien (Morocco) - Rabat

Instituto Tecnologico de Aeronautica (Brazil) - São José dos Campos

Department of Scientific and Industrial Research (Great Britain) - Singapore, Malaya

The assistance of the station operators and other personnel of these agencies in obtaining the data contained in this report is gratem fully acknowledged. 
The following publications contain additional information on radio noise:

1. W. Q. Crichlow, D. F. Smith, R. N. Morton, and W. R. Corliss, "Worldwide Radio Noise Levels Expected in the Frequency Band 10 Kilocycles to 100 Megacycles," NBS Circular 557, August 25, 1955. 2. "Report on Revision of Atmospheric Radio Noise Data," C. C.I.R. Report No. 65, VIIIth Plenary Assembly, Warsaw, 1956 (International Radio Consultative Committee, Secretariat, Geneva, Switzerland).

3. A. D. Watt and E. L. Maxwe11, "Measured Statistical Characteristics of VIF Atmospheric Radio Noise," Proc. IRE, 45, 1 , 55 (1957).

4. W. Q. Crichlow, "Noise Investigation at VLF by the National Bureau of Standards," Proc. IRE, 45, 6, 778 (1957).

5. A. D. Watt and E. L. Maxwell, "Characteristics of Atmospheric Noise from I to $100 \mathrm{kc}$ " Proc. IRE, 45,6, 787 (1957).

6. F. F. Fulton, Jr., "The Effect of Receiver Bandwidth on Amplitude Distribution of V.L.F. Atmospheric Noise," National Bureau of Standards, VLF Symposium Paper 37, Boulder, Colorado, 1957.

7. H. E. Dinger, "Report on URSI Commission IV - Radio Noise of Terrestrial Origin," Proc. IRE, 46,7, 1366 (1958).

8. A. D. Watt, R. M. Coon, E. L. Maxwell, and R. W. Plush, "Performance of Some Radio Systems in the Presence of Thermal and Atmospheric Noise," Proc. IRE, 46, 12, 1914 (1958).

9. W. L. Taylor and A. G. Jean, "Very-Low-Frequency Radiation Spectra of Lightning Discharges," NBS J. of Research-D. Radio Propagation, 63D, 2, 199 (1959).

10. W. Q. Crichlow, C. J. Roubique, A. D. Spaulding, and W. M. Beery, "Determination of the Amplitude-Probability Distribution of Atmospheric Radio Noise from Statistical Moments," NBS J. Research-D. Radio Propagation, 64D, 1, 49 (1960).

11. Tatsuzo Obayashi, "Measured Frequency Spectra of Very-LowFrequency Atmospherics," NBS J. of Research-D. Radio Propagation, $64 \mathrm{D}, 1,41$ (1960)。 
Data included in this report and the standard time for each station are as follows:

\begin{tabular}{|c|c|c|c|}
\hline Station & Data & $\begin{array}{l}\text { Time } \\
\text { Zone }\end{array}$ & $\begin{array}{l}\text { To Convert } \\
\text { LST to GMT } \\
\text { (hours) }\end{array}$ \\
\hline Balboa & June July August 1959 & $75 \mathrm{~W}$ & +05 \\
\hline Bill & June July August 1959 & $105 \mathrm{~W}$ & +07 \\
\hline Boulder & June July August 1959 & $105 \mathrm{~W}$ & +07 \\
\hline Byrd Station & June July August 1959 & $120 \mathrm{~W}$ & +08 \\
\hline Cook & June July August 1959 & $135 \mathrm{E}$ & -09 \\
\hline Enkoping & June July August 1959 & $15 \mathrm{E}$ & -01 \\
\hline Front Royal & June July 1959 & $75 \mathrm{~W}$ & +05 \\
\hline Tbadan & June July 1959 & GMT & 0 \\
\hline Kekaha & June July August 1959 & $150 \mathrm{~W}$ & +10 \\
\hline Ohira & June July August 1959 & $135 \mathrm{E}$ & -09 \\
\hline Pretoria & June July August 1959 & $30 \mathrm{E}$ & -02 \\
\hline Rabat & June 1959 & GMT & 0 \\
\hline São José dos Campos & June July August 1959 & $45 \mathrm{~W}$ & +03 \\
\hline Singapore & June July August 1959 & $105 \mathrm{E}$ & -07 \\
\hline Thule & June July August 1959 & $75 \mathrm{~W}$ & +05 \\
\hline
\end{tabular}

Previous data from the NBS World-Wide Network have been published in the following Technical Note 18 series:

18-1 July 1, 1957 - December 31, 1958

18-2 March, April, May 1959 
MOST PROBABLE AND MINIMUM VALUES OF $L_{d}$ VERSUS $V_{d}$ FOR ATMOSPHERIC RADIO NOISE

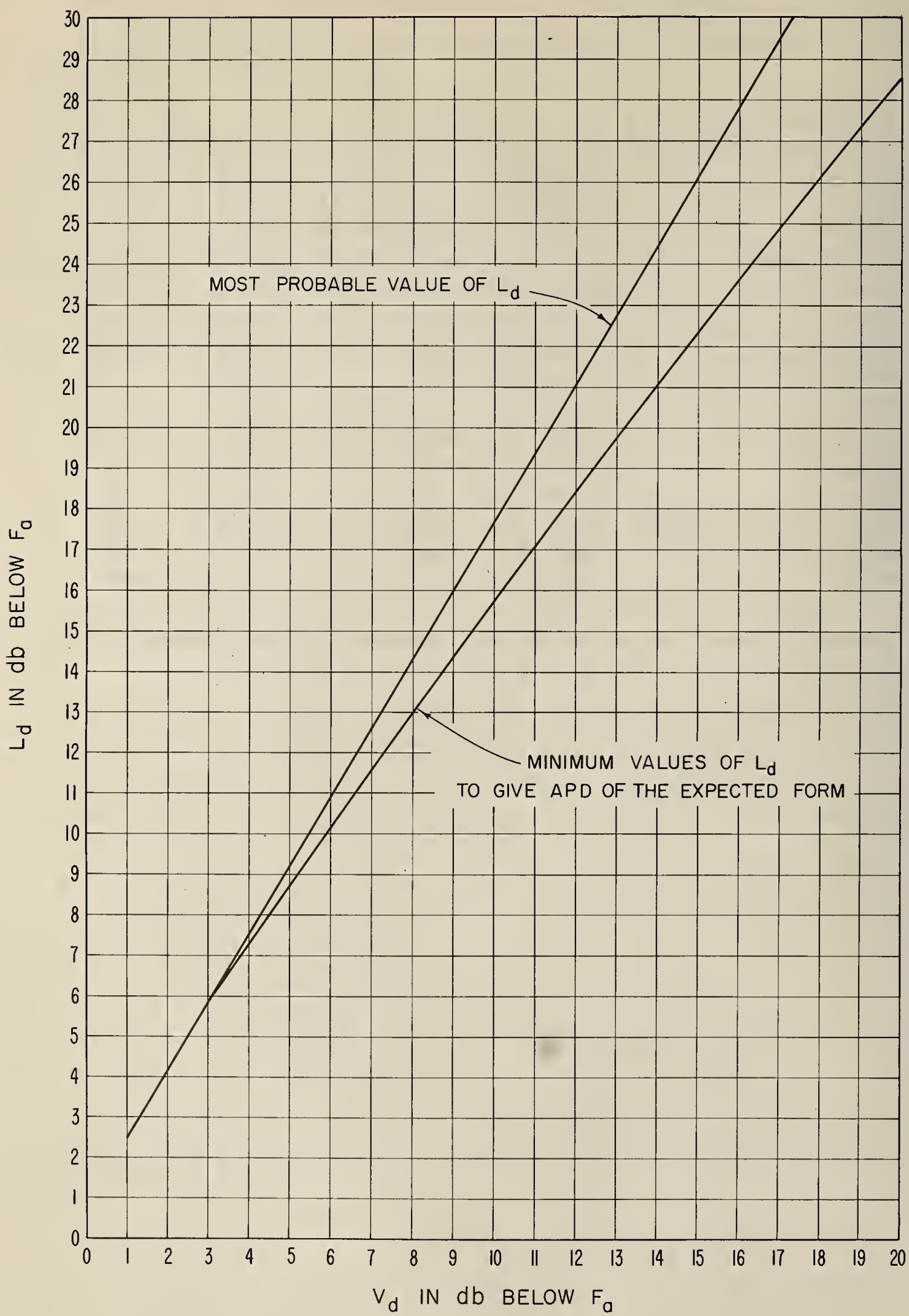




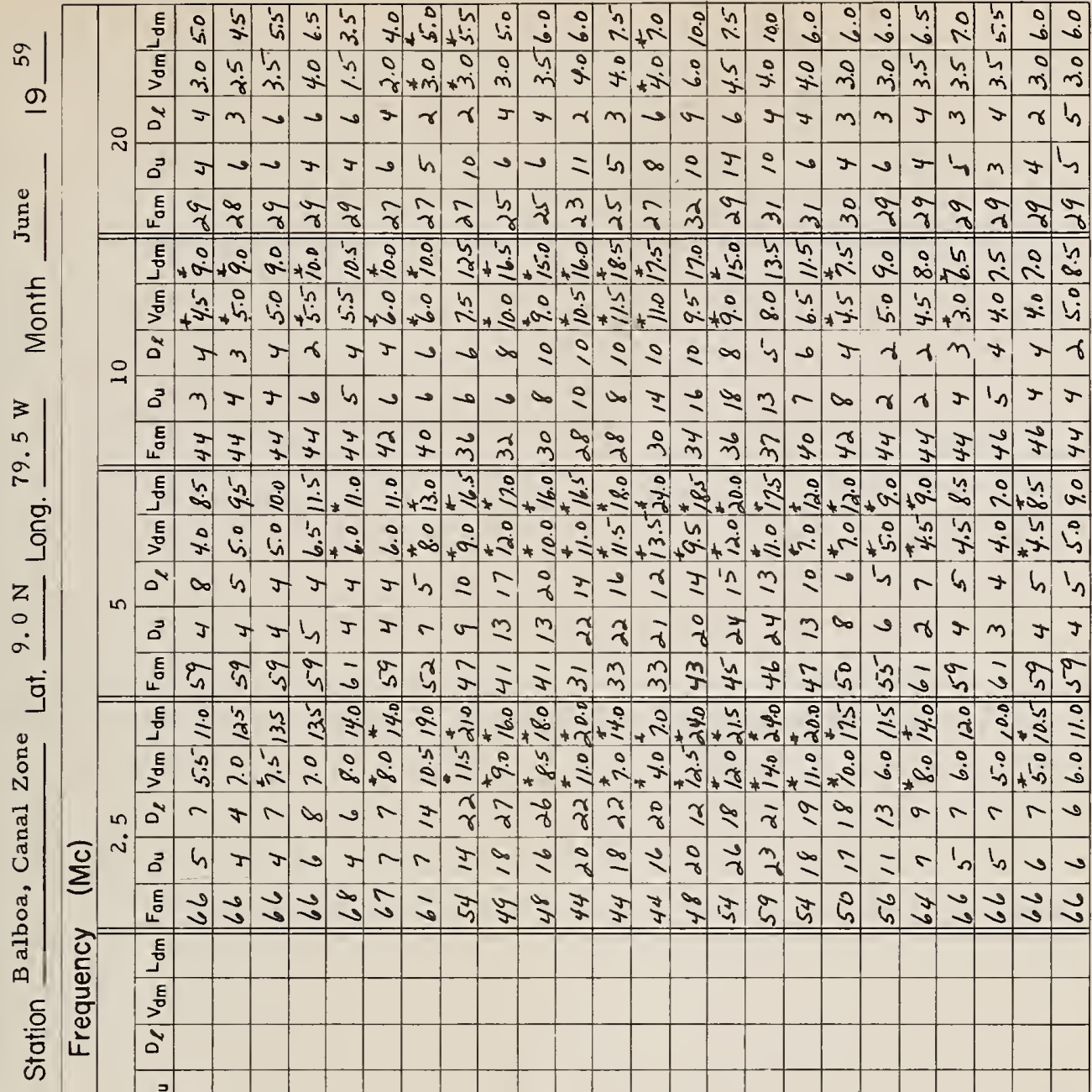

$0^{3}$

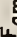

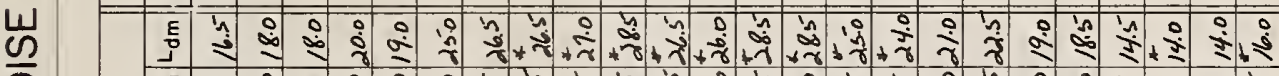

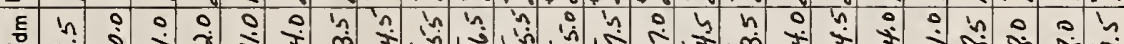

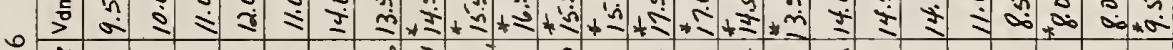

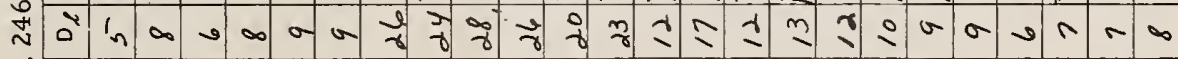

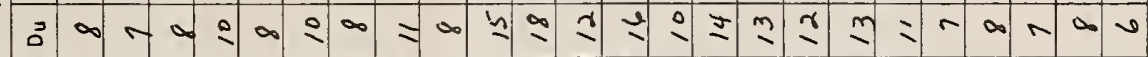
ह

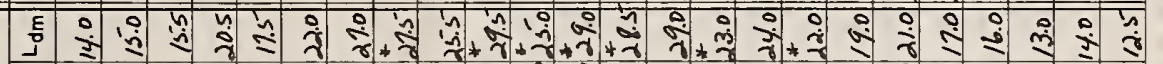
攼

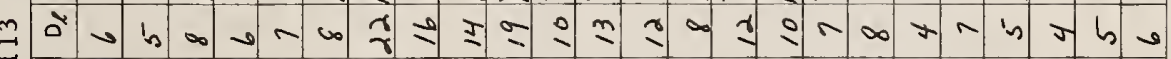

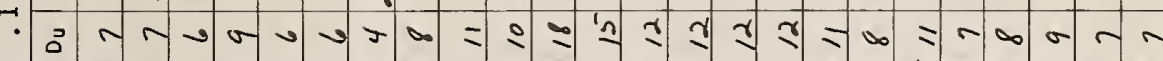

है

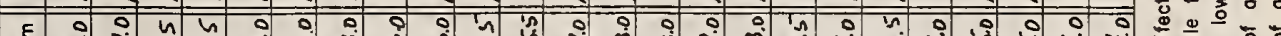

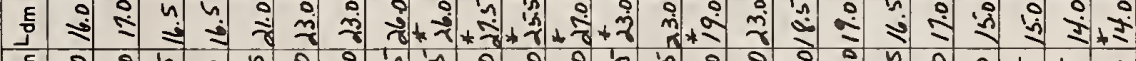

है

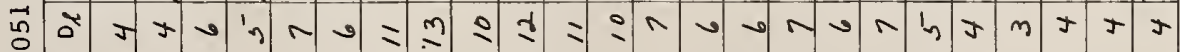

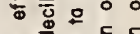

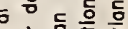

突

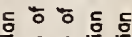

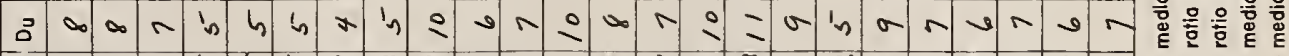

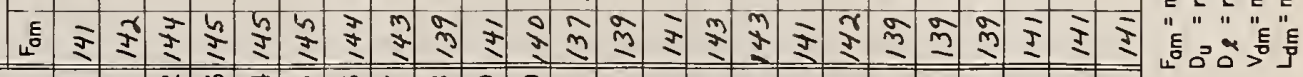
(LS7) $\mathrm{AnOH}$ : 
告

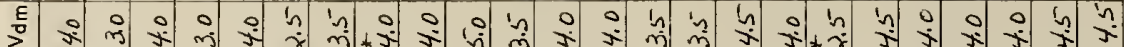

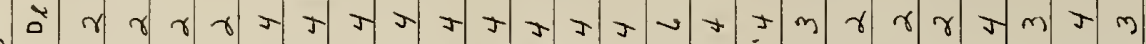

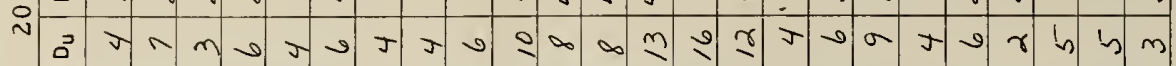

E

E 0 : 8 in

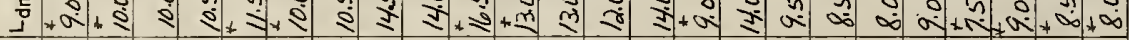

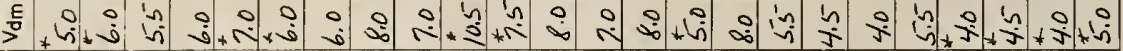

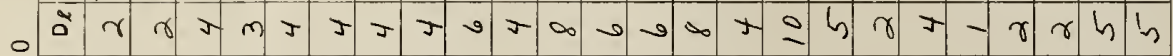

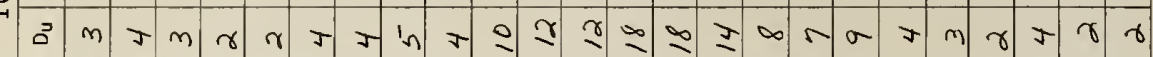

ह

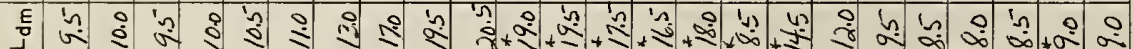
> ๑

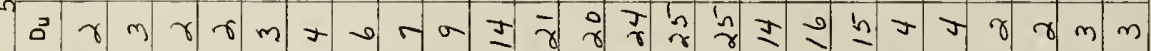

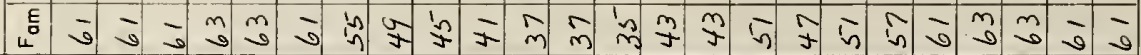

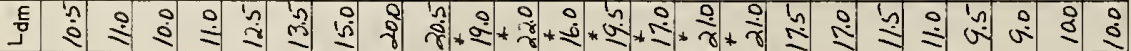

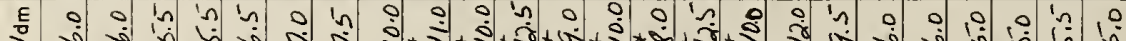

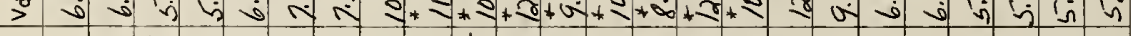

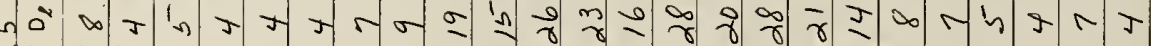

窃

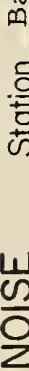

宸 E

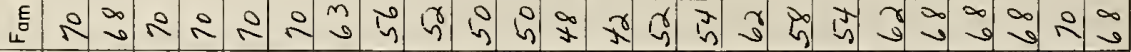
ठै

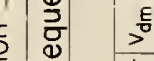

它

ธั

$0^{3}$

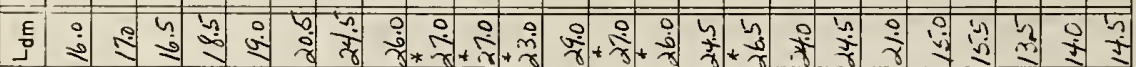
ह

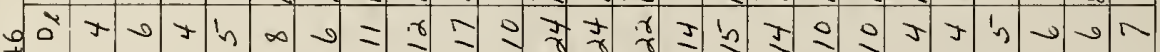
y.

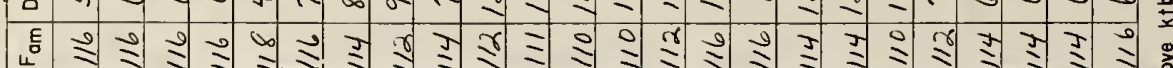

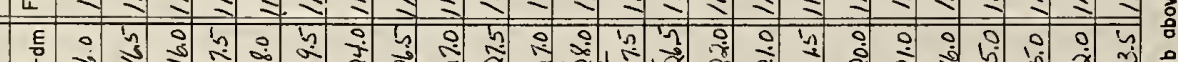

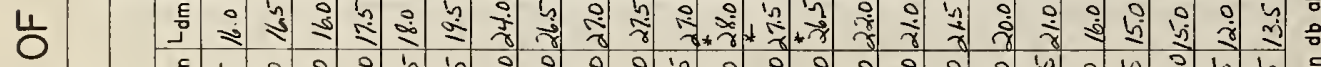

节

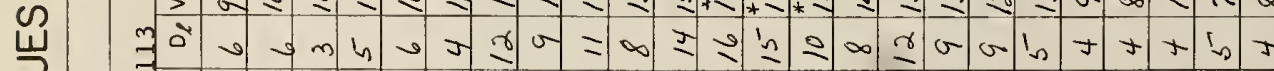

$\frac{1}{5}$

a

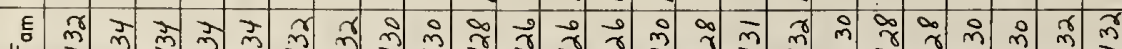

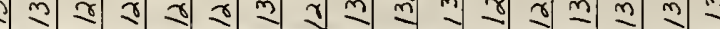

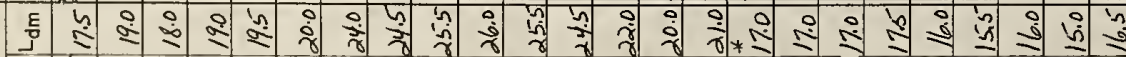

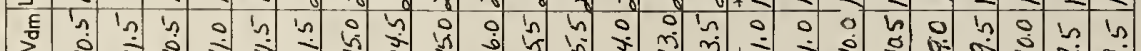

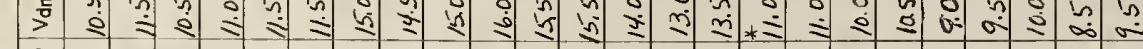

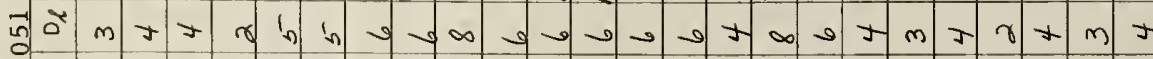


E[

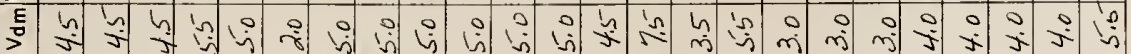

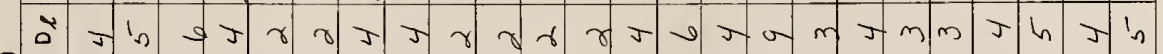

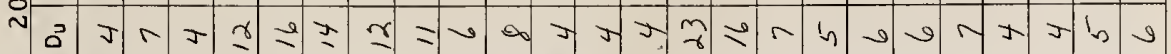

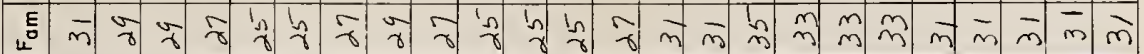

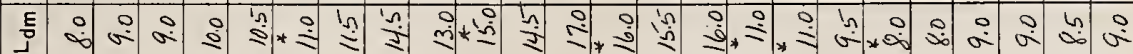
E 0 in 0 o in 0 o 0 in 0 in 0 o in o

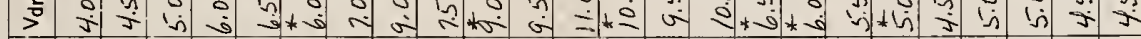

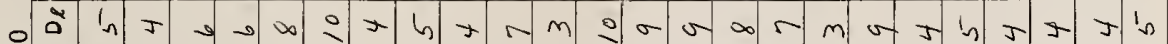

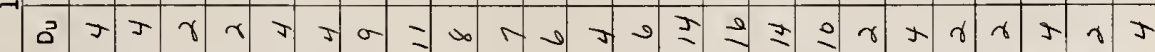
3
2
2
7 E का

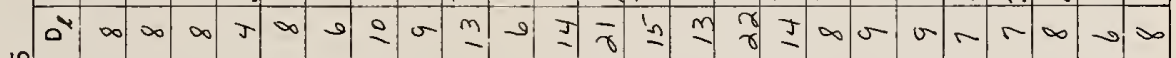

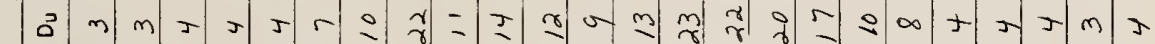

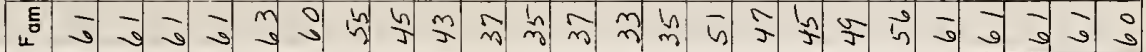

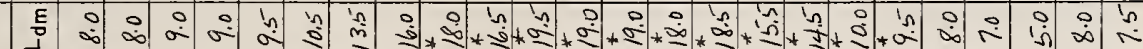

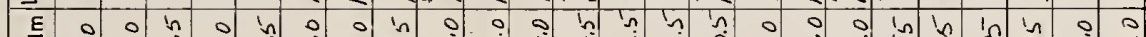

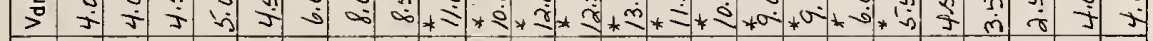

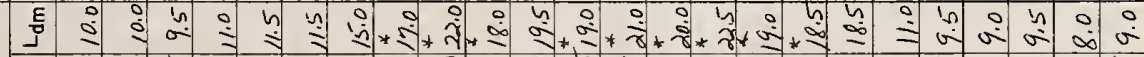

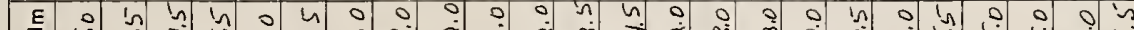

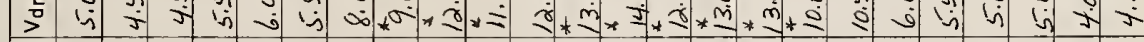
ก๊

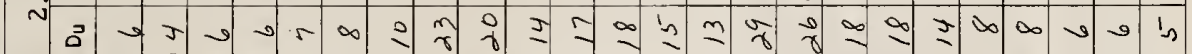

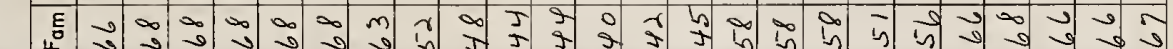

\begin{tabular}{|l}
\hline 5 \\
\hline 5 \\
\hline 5 \\
\hline 0 \\
\hline 0
\end{tabular}

$a^{3}$

E

W

+ 2 , 2 - 2 E

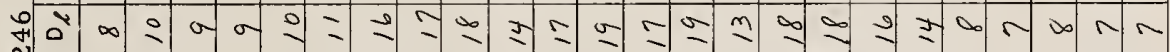
ㄴ.

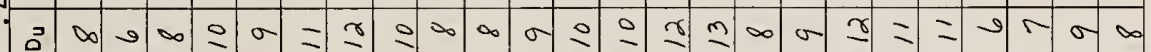
ह ह

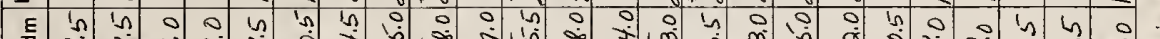

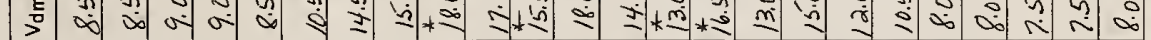

岂

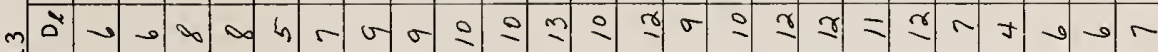

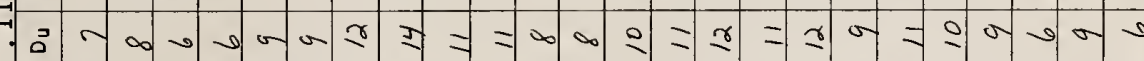
E $m m$ m $m$ m mm

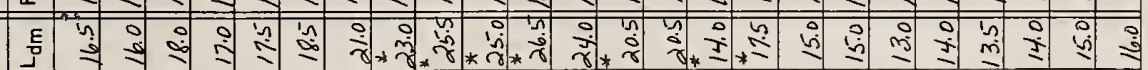
है ही

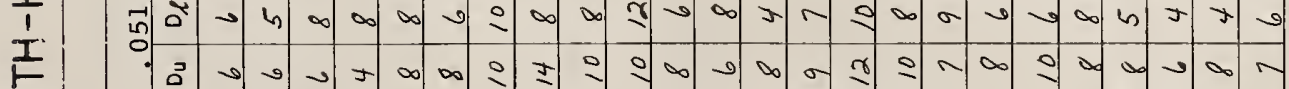

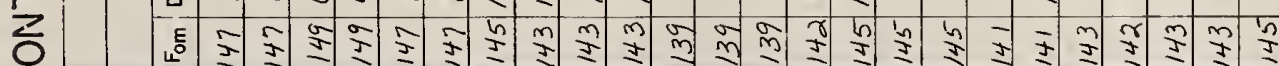

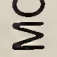
(IS7) $\operatorname{MOH}$ : 


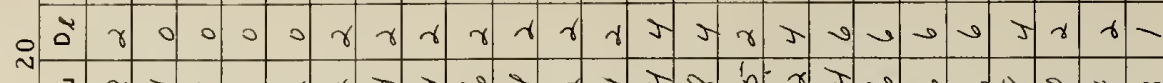

苟

$+\infty$ \& $\rightarrow m$

斿

产

:

乌்

z

$\stackrel{\sim}{\sim}$

苛

E

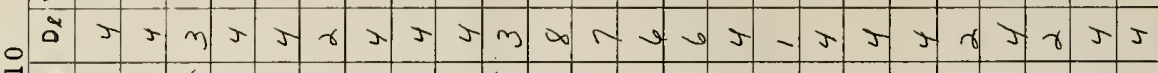

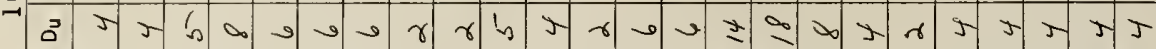

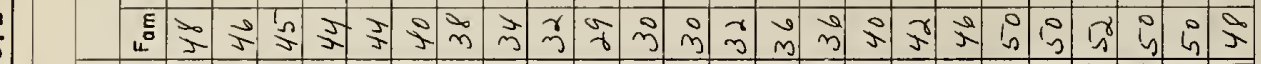

$\stackrel{\mathcal{J}}{\mathrm{J}}$

政

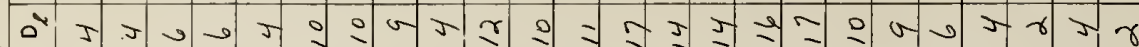

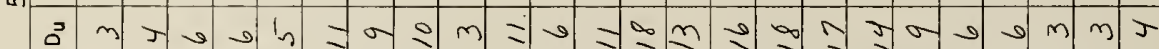

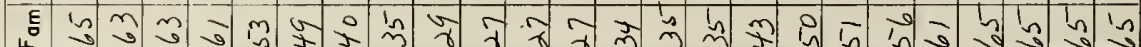
통

E

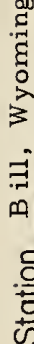

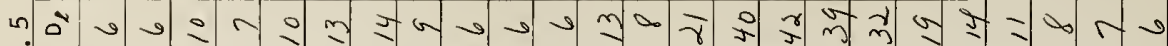

$\underline{0}$

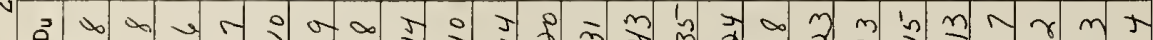
है

도 몽

흥

$>^{\frac{E}{0}}$

in

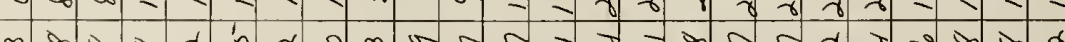

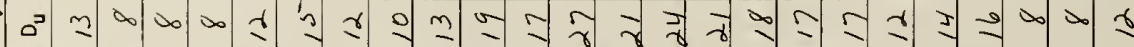

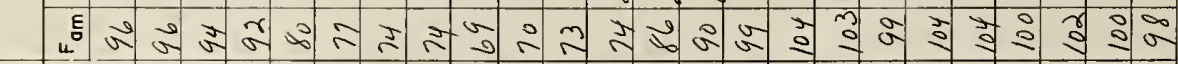

$\frac{w}{2}$

E

틈

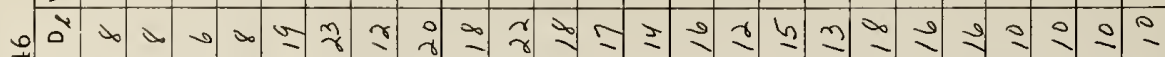

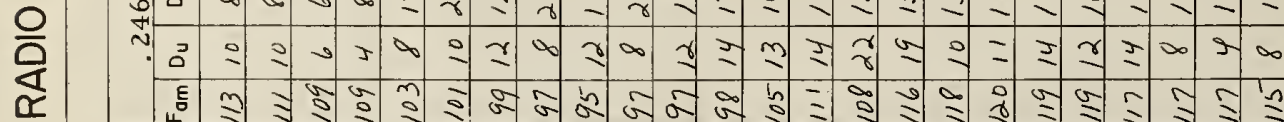

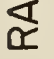

แั

$=$

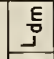

$\stackrel{5}{>}$

岁

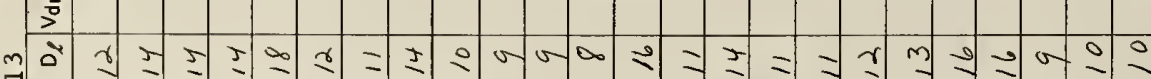

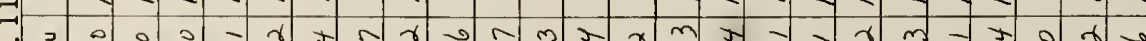

. ว

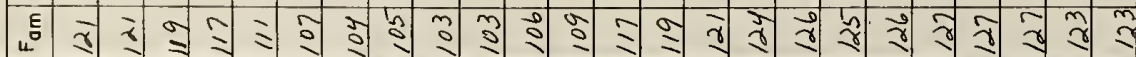

$\frac{0}{2}$
$\frac{0}{1}$
$\frac{1}{I}$
$\frac{1}{5}$
$\frac{1}{2}$

E⿱一彑口丂

E

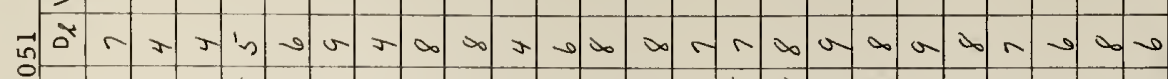

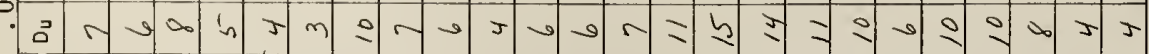

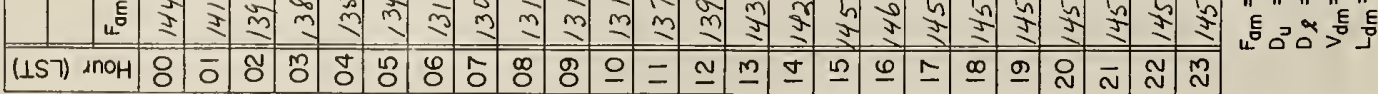




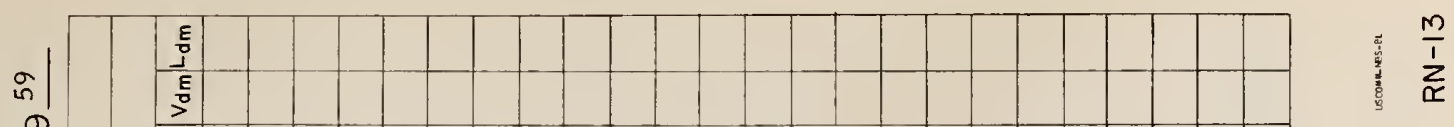

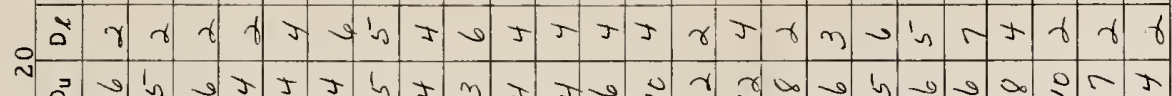

寻

$\frac{c}{\stackrel{c}{c}}$

ปे

है

E

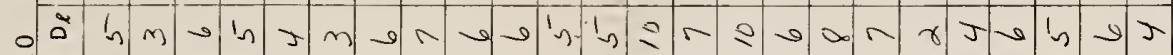

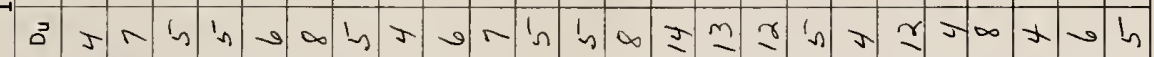

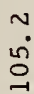

¿ุ

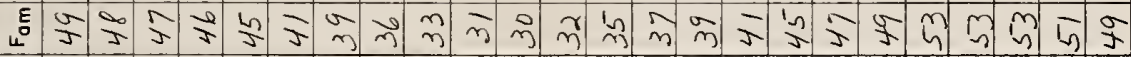

E

$\stackrel{E}{>}$

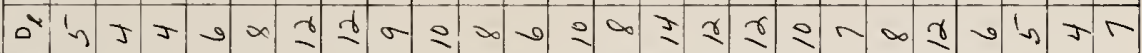

$z$
$\sim$
$\dot{y}$
$\dot{m}$

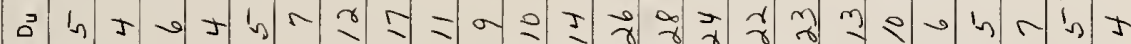

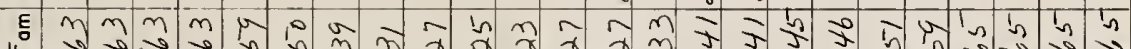

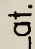

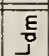

E

苟

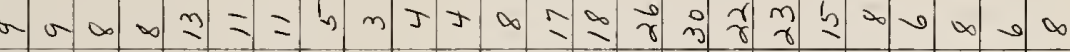

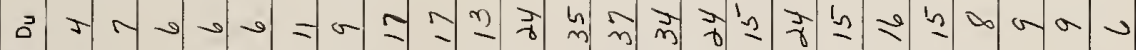

ह

ठํ.

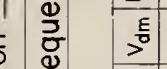

离

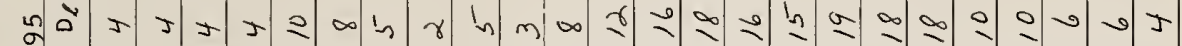

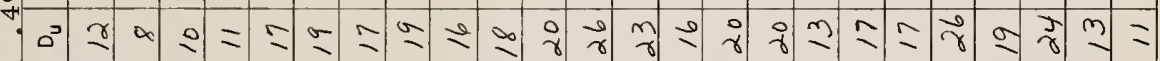

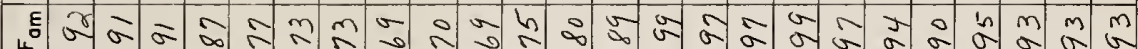

$\frac{1}{2}$

宩

통

एक

울

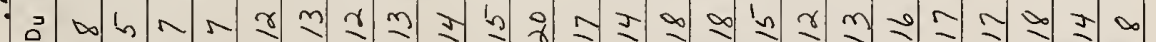

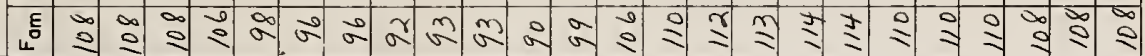

हᄐ

แ

岁

E

$\stackrel{5}{>}$

-

(1)

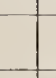

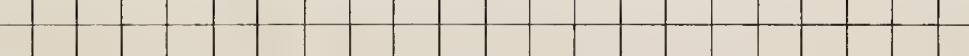

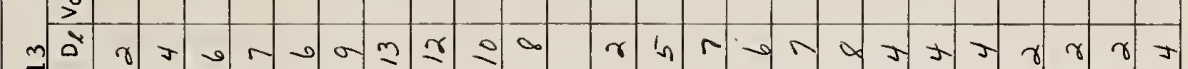

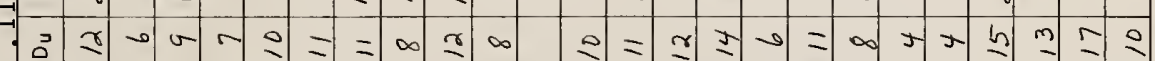

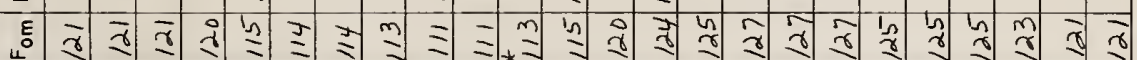

$+$

E⿱一⿻口卄口

$\frac{0}{\frac{0}{1}}$

틍

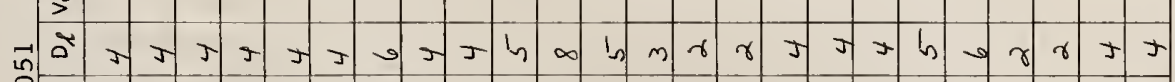

I

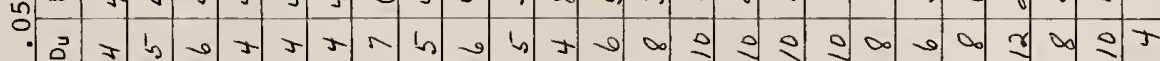

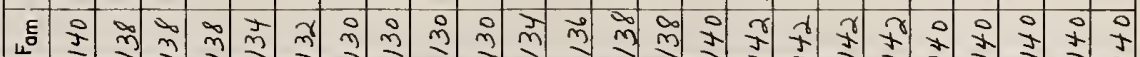

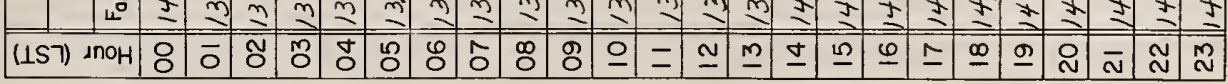

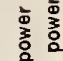

芩

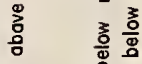

음 용

드

응응응

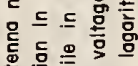

ह

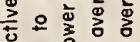
要

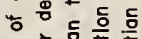

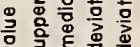

든 드음

흫읗읗흫ㅎㅎㅌ흩

"E" " " "ह 퉁 


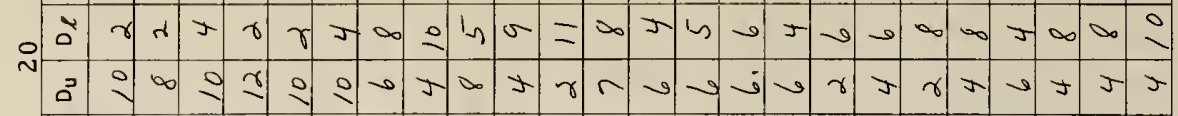

$\begin{aligned} & u \\ & 0 \\ & 5 \\ & 00 \\ & 5 \\ & 4\end{aligned} \mid$

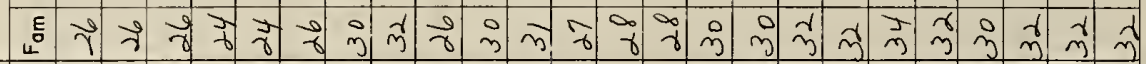

音

咅

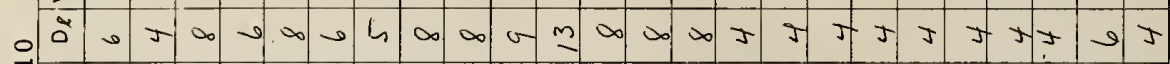

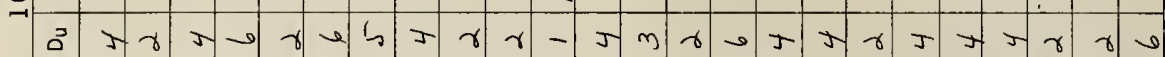

บั

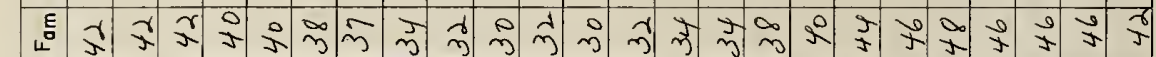

익

פं

$\frac{E}{5}$

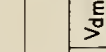

z.

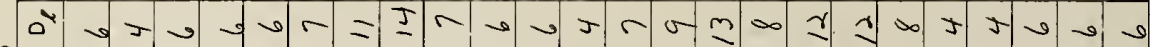

$\dot{q}$

过

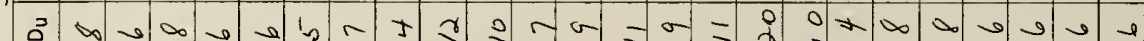

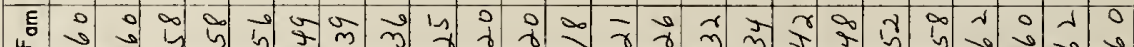
E

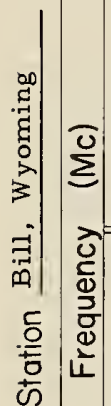

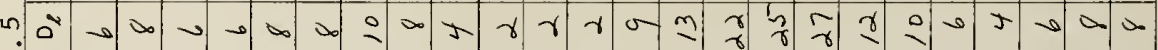

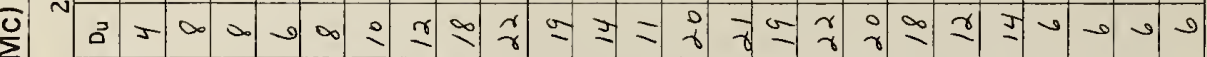

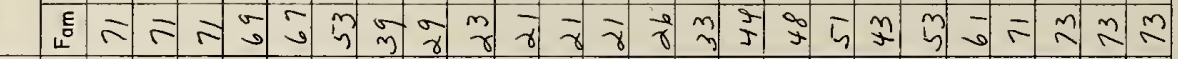

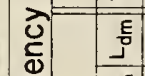

要

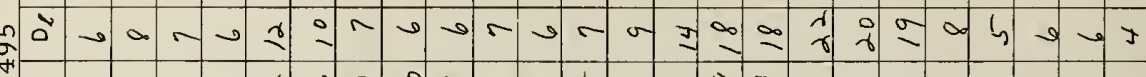

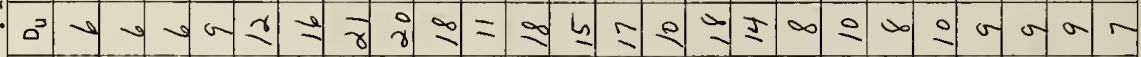

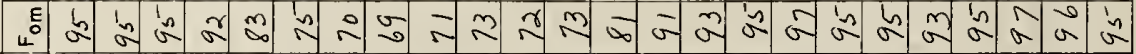

$\frac{\mathrm{w}}{\mathrm{O}}$

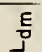

竞

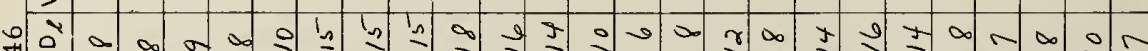

일

N

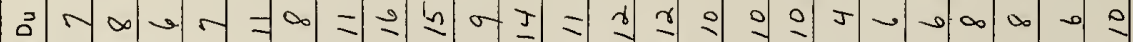
ह ज० $\frac{E}{5}$

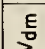

$\stackrel{\stackrel{m}{3}}{\stackrel{3}{5}}$

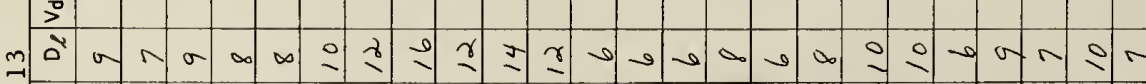

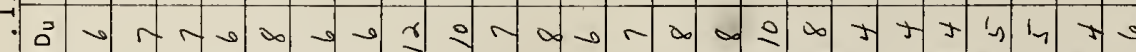

ผ

똑

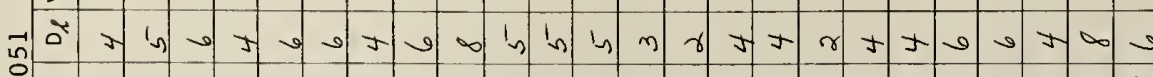

I

E

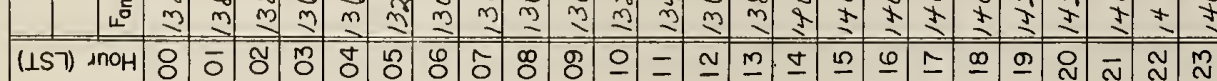

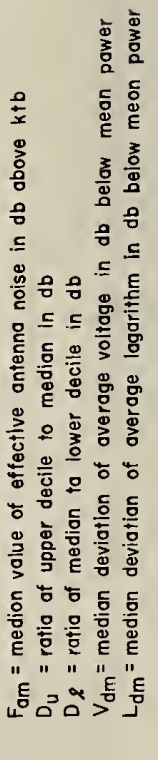


in

$\stackrel{0}{\stackrel{5}{5}}$

동

3
-1
0
0

家

Z

$\stackrel{-1}{\circ}+$

高

웅

음 立絃

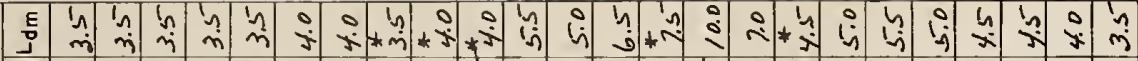

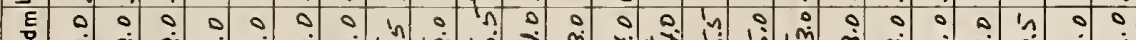

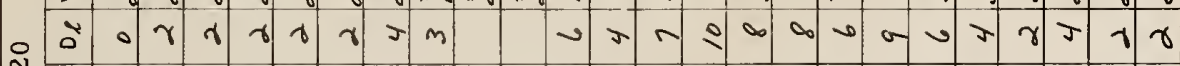

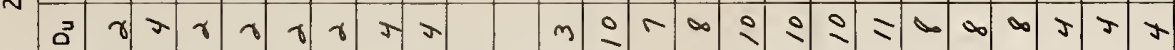

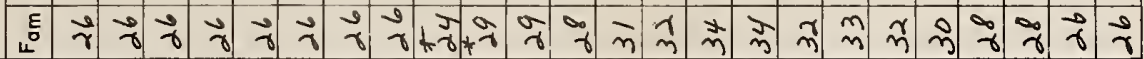

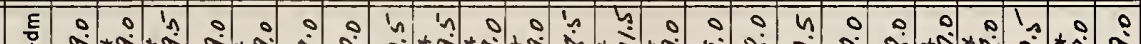

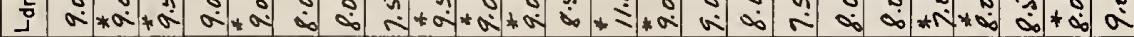

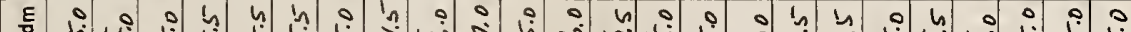

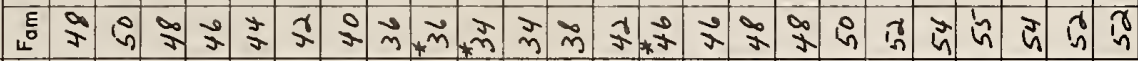
ப

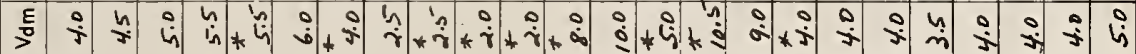

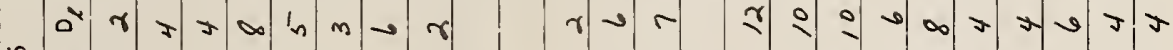

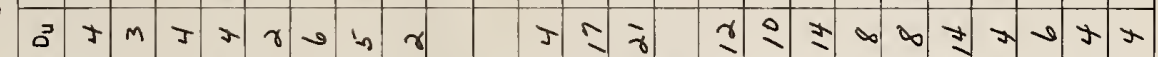

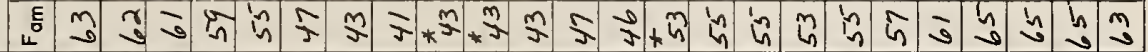

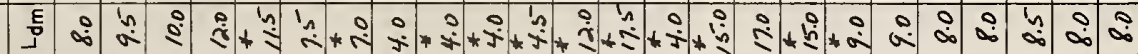

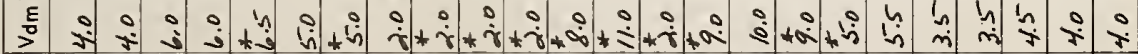

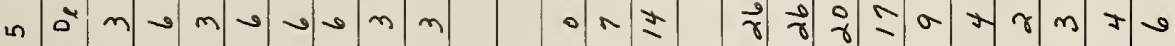

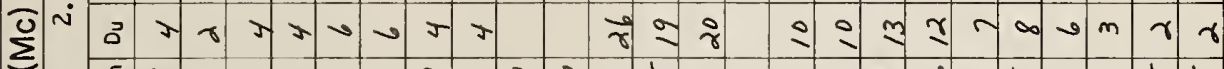
Eั

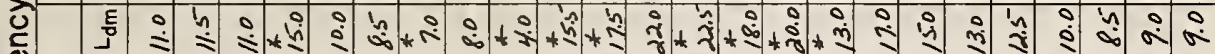

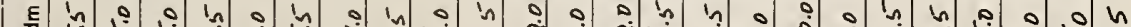

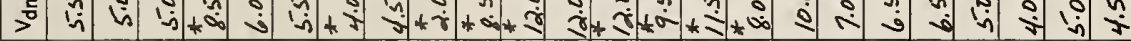

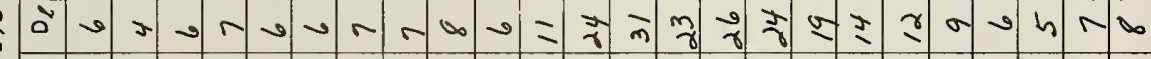

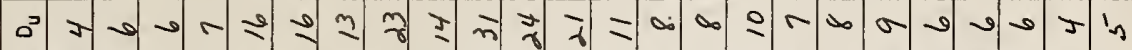

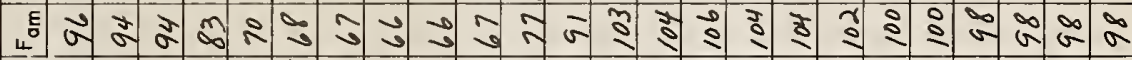

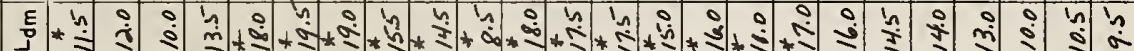

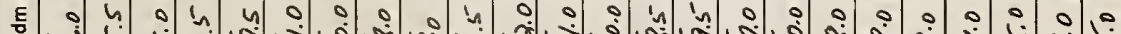

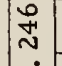

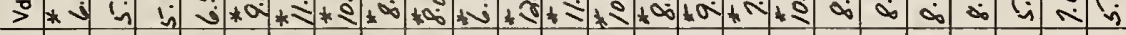

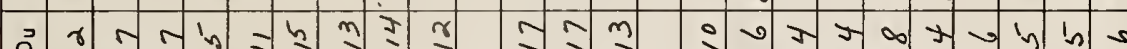

ह

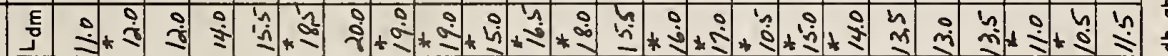

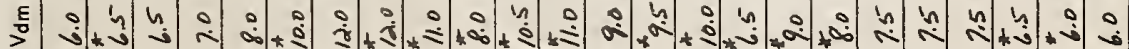

崩

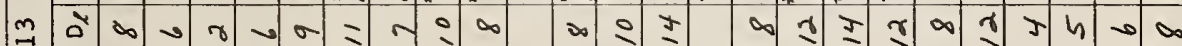
$\rightarrow$

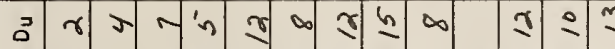

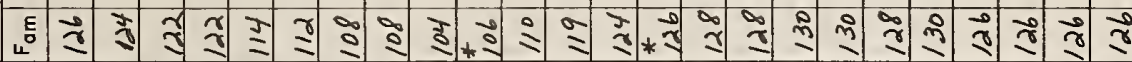

Е

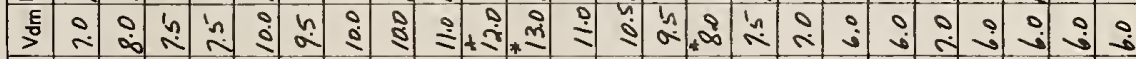

में 0

כ

$\infty$ o $9 \cos$ 


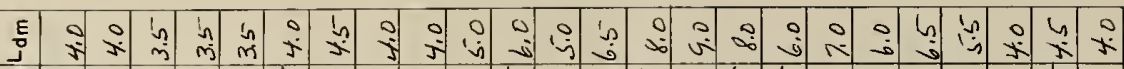

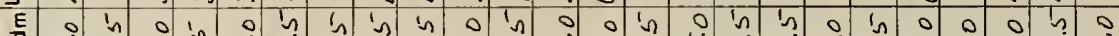

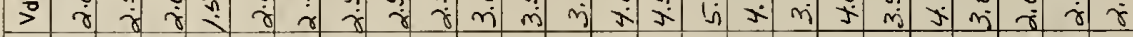

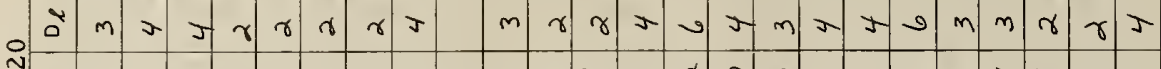

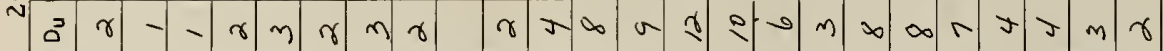

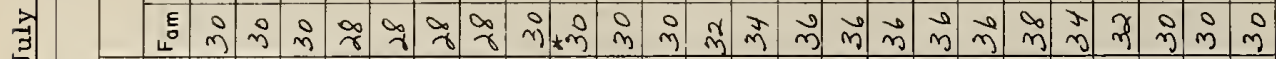

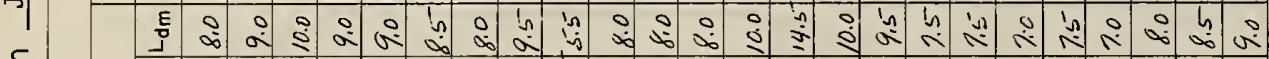
F

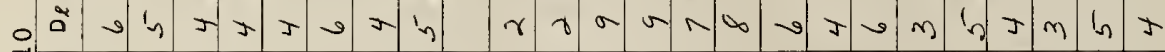

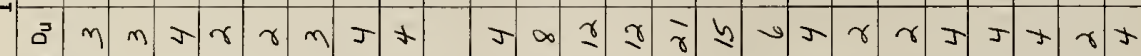

일.

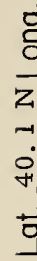

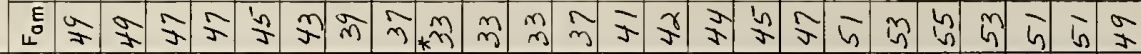

E 00 o in $\ln 0$ O

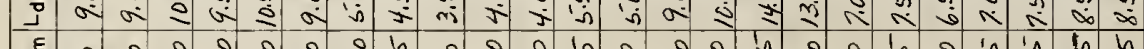

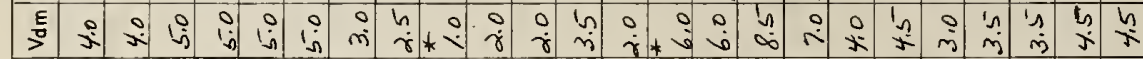

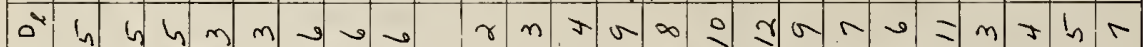

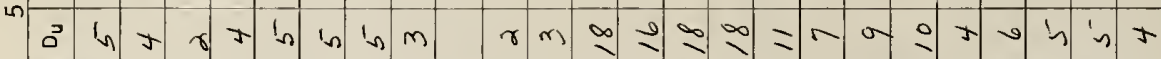
E - ज约

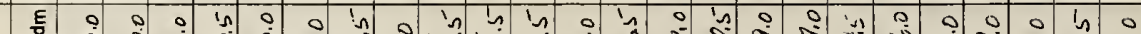

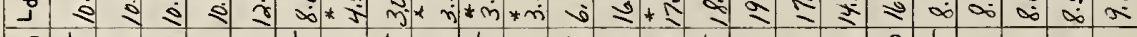

镸

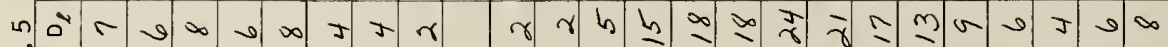

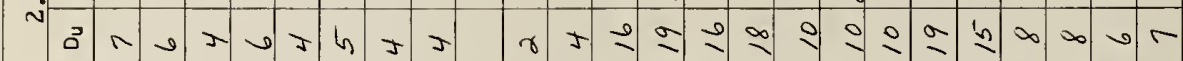
है

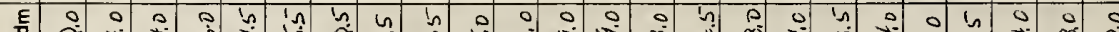

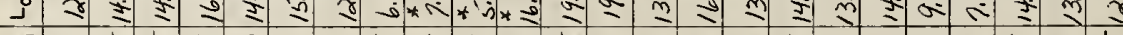

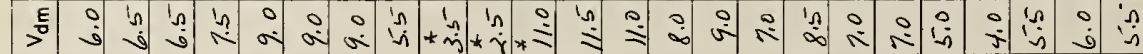

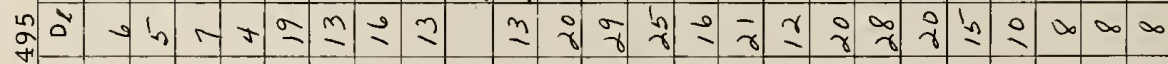
व $\infty$ J J «

$\frac{w}{2}$

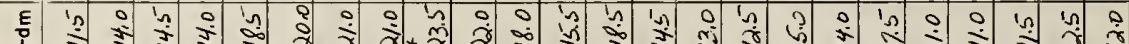

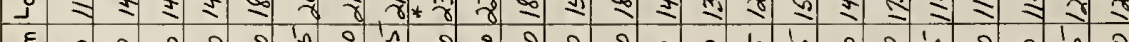
孠 8.

웅

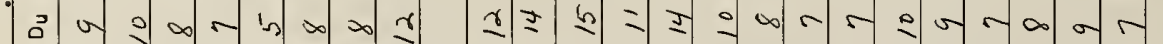

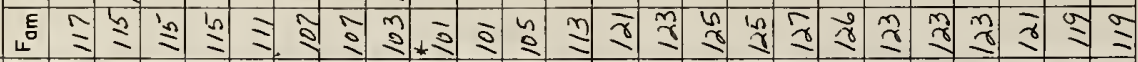

ร

E

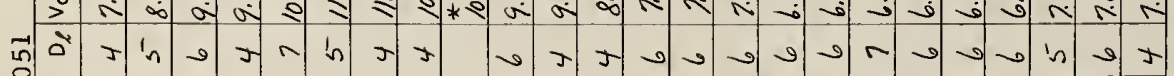

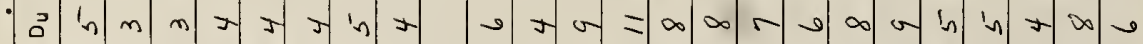

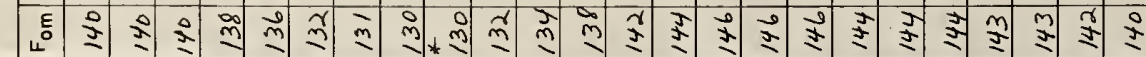

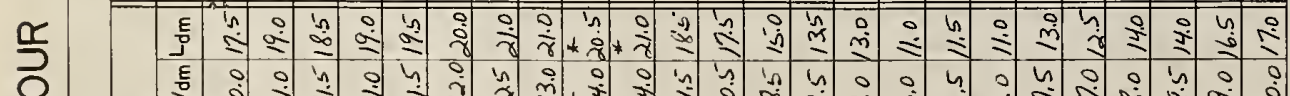

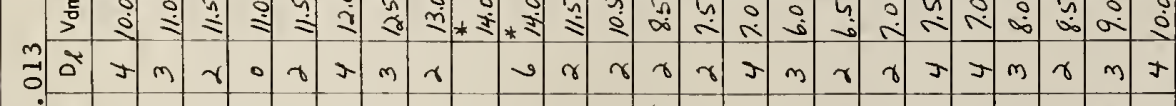

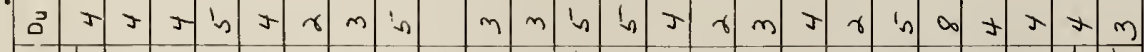


E

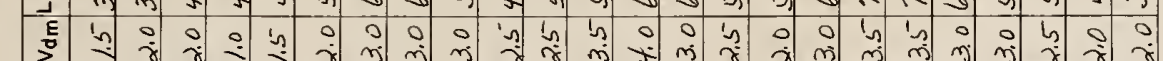

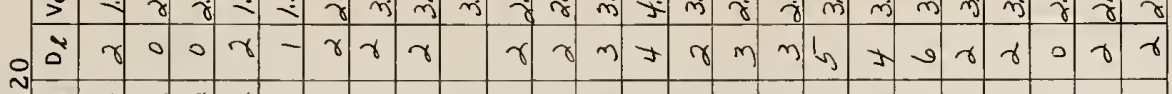

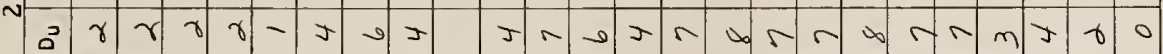

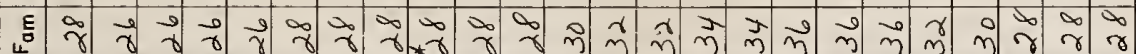

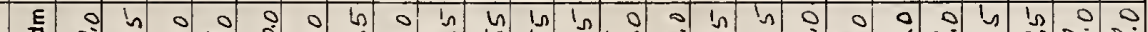

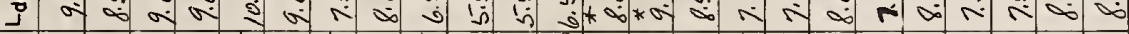

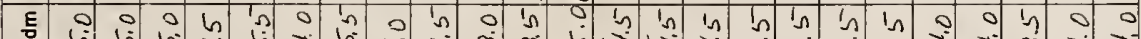

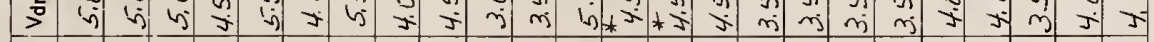

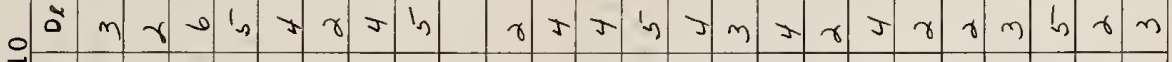

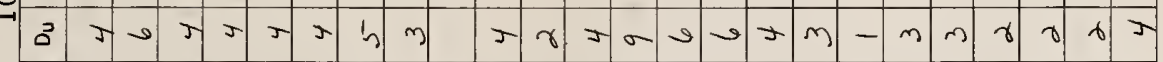

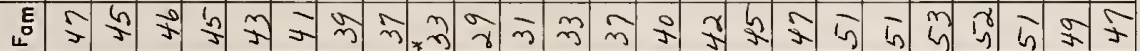

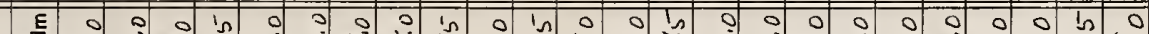

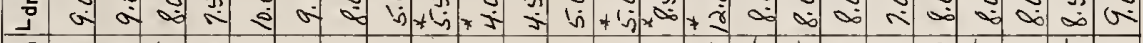

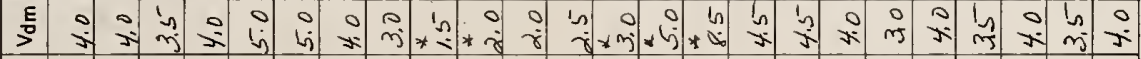

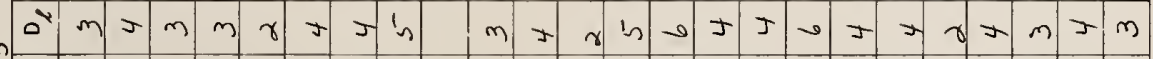

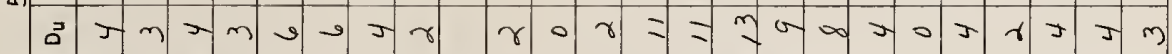

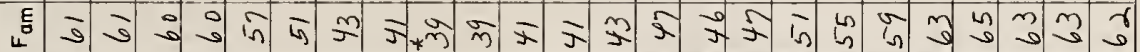

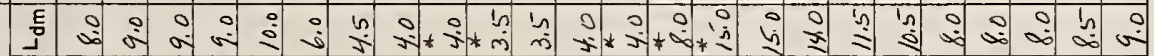

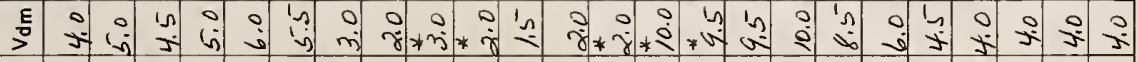

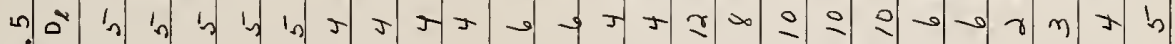

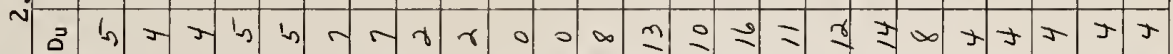

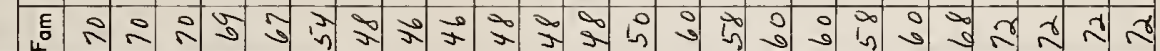

ह

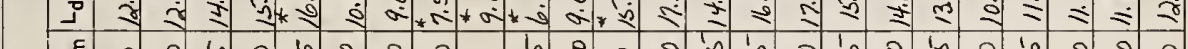
言 立 紋

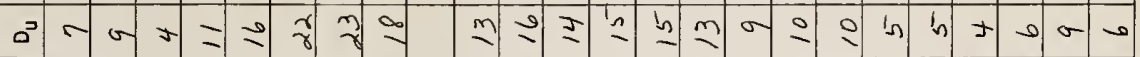

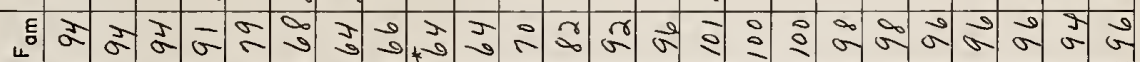

$\frac{w}{\mathrm{O}}$

울.

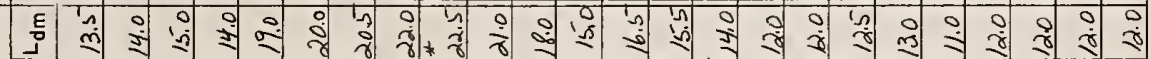

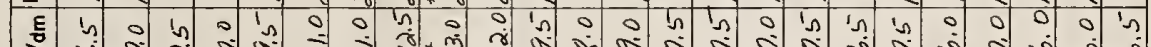

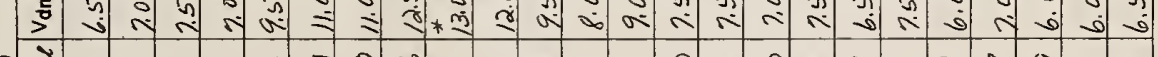

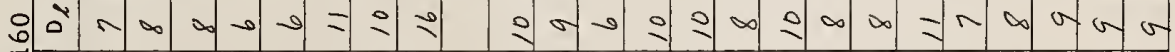

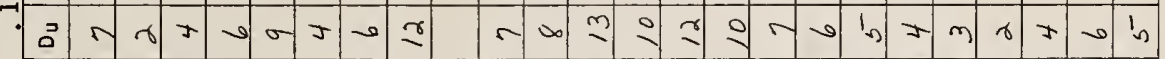

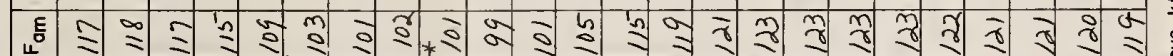
텀 商 E 00 - 0 जी

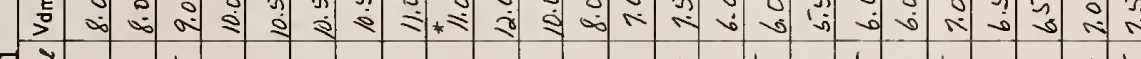

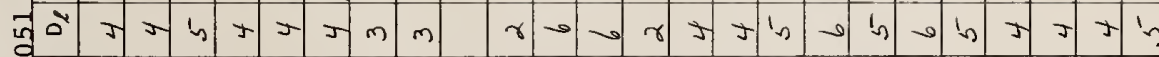

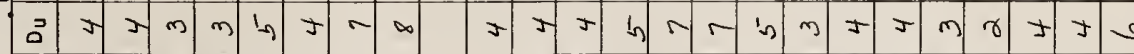

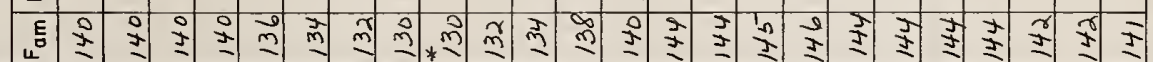

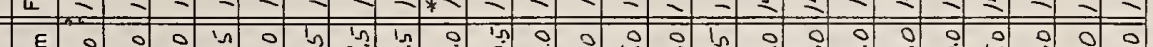

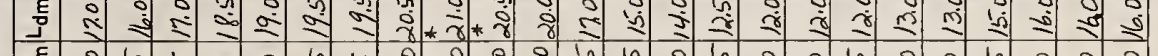
兵

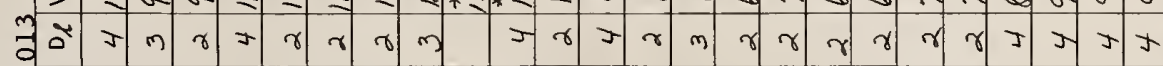
ja

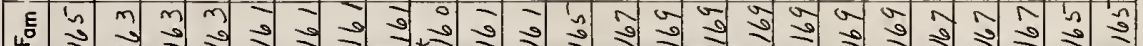
듬 용

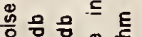
도.음

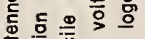
要 总 :

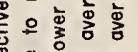
홍등드

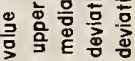
등ㄴㅎㅇ 등

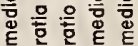

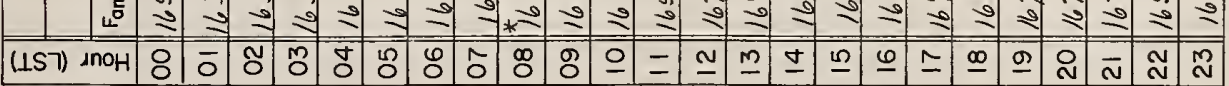




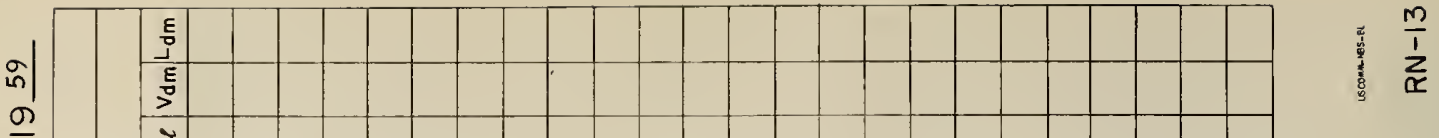

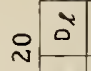

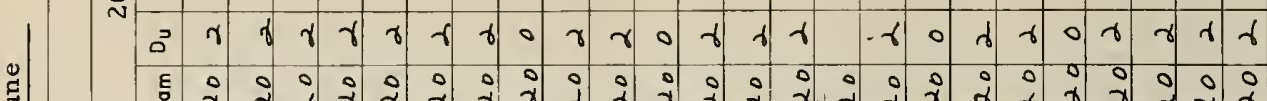

点.

$\stackrel{5}{\frac{5}{5}}$

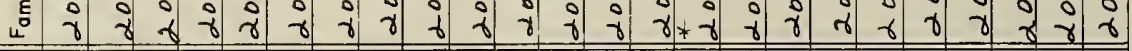

E

>

$\circ$

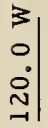

a

סु

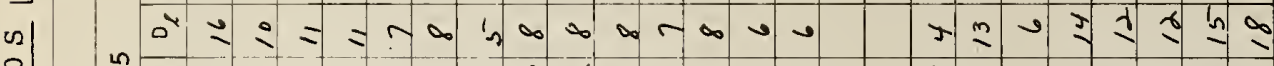

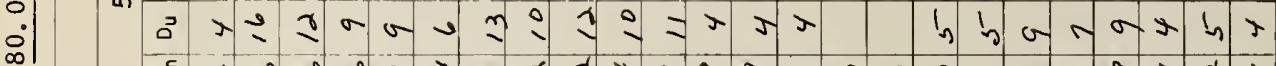

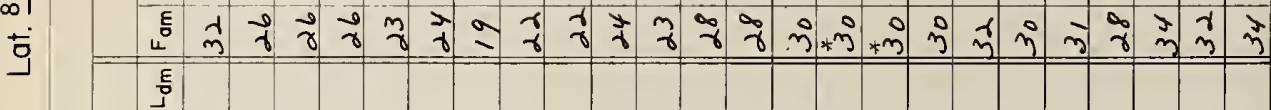

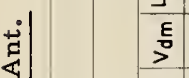

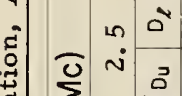

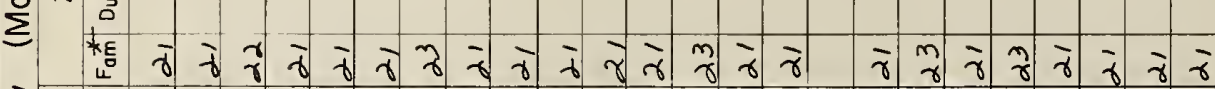

究

ᄃิ 蛋

范

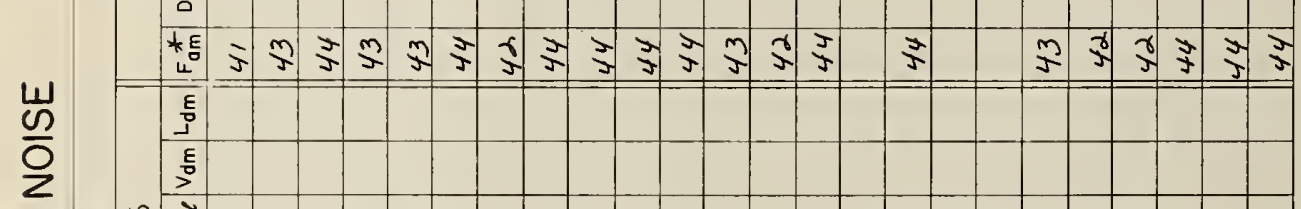

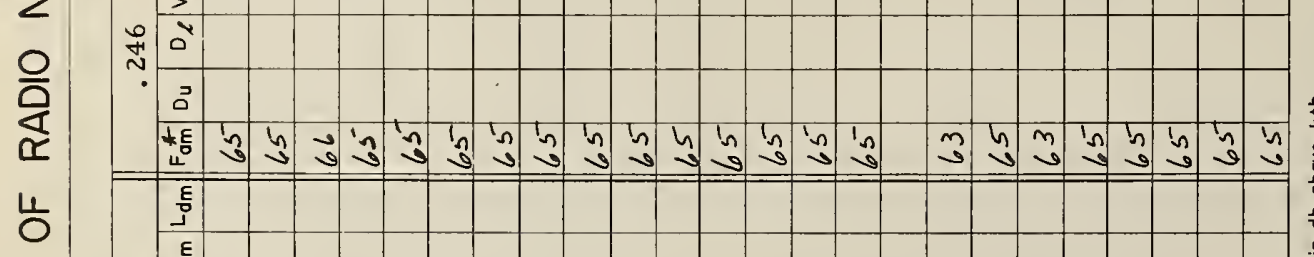

$\geqslant$

$0>0 . r x y .40$

$\gamma 0 \gamma r \gamma-0$ 당

$=\begin{array}{lllll}z & x & 4 & x\end{array}$

$\because 5 m m x$

r.

*rmam $+>$

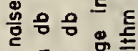

드드 몽흔

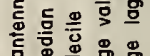

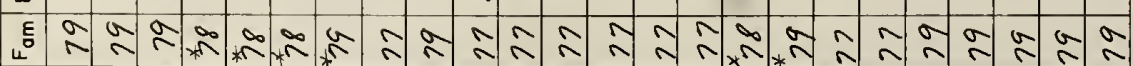

E

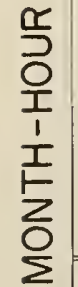

\begin{tabular}{ll}
\hline$\frac{E}{3}$ \\
\hline$\frac{5}{5}$ \\
\hline 5
\end{tabular}

मूर

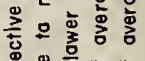

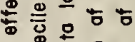

t5 5 드음

음 응 흥 흥 흘

등

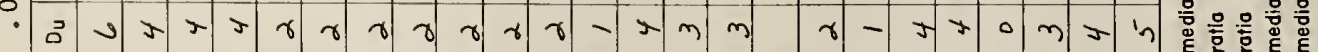

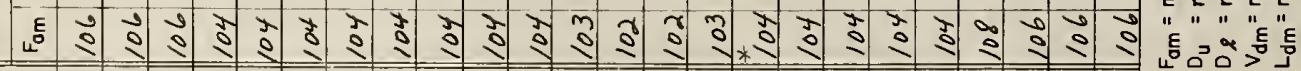

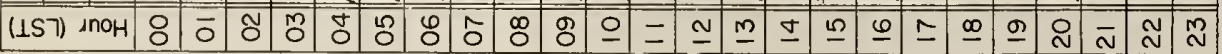




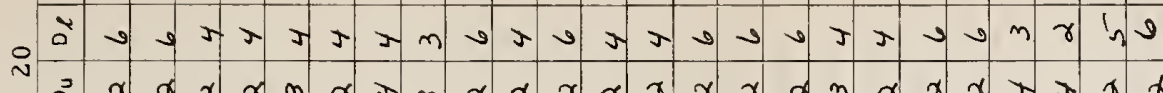

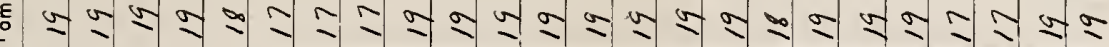

통

통

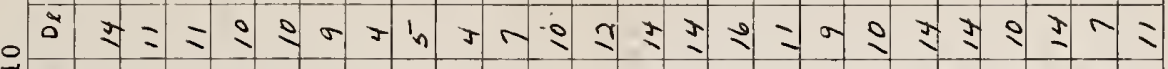

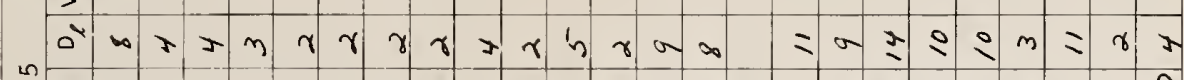

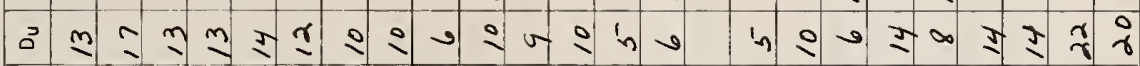

के

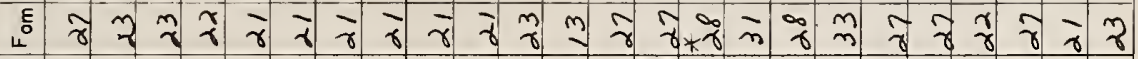

है

ह

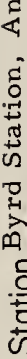

in

$\gamma \gamma \gamma \eta \gamma>\gamma$

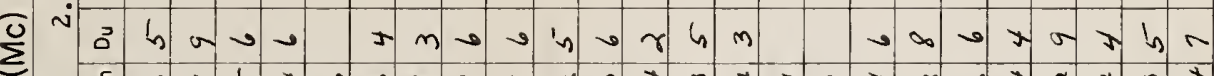
E

ठे

$\stackrel{0}{\frac{D}{2}}$

एँ

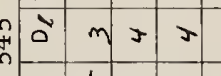

$\gamma \gamma \gamma \gamma \gamma \gamma \gamma$

$\lambda 2 \pi \sin 2 \pi$

obr

$\gamma \gamma \gamma+\gamma$

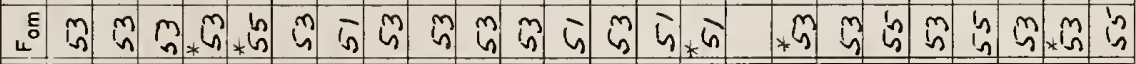

鲁

E

है

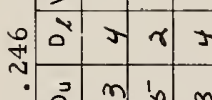

$\lambda \gamma^{\gamma} \gamma^{\gamma} \gamma^{\gamma}$

울

ฟ $\gamma \gamma \gamma \gamma \gamma \gamma \neq$

है

$\gamma m \gamma$ in $m \gamma r$

틈

$\stackrel{4}{0}$

莺

E

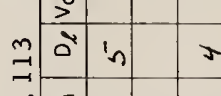

$m x y>2 y+6$

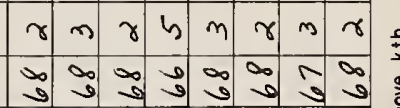

产言

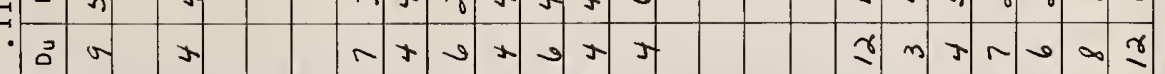

เ

틈

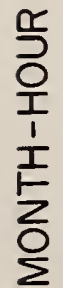

至

요을

एह

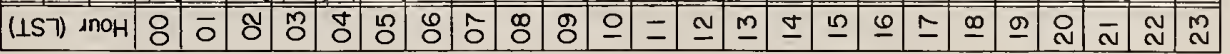




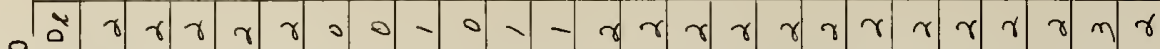

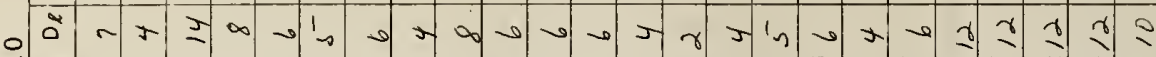
$>$

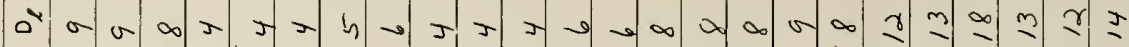

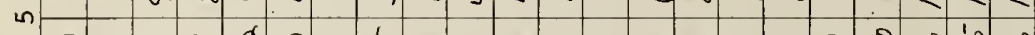
उजa

है जु की

峞

E

E

$>$

高

齐

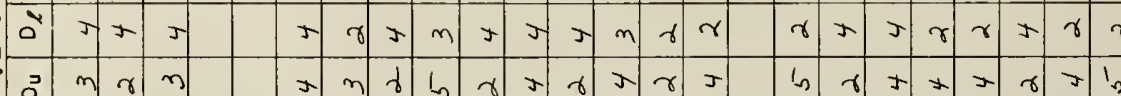

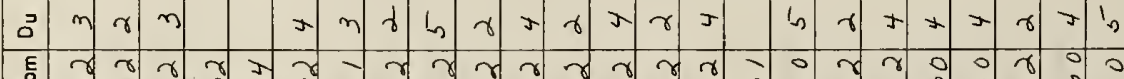
ผ E

낭

E

岂

m

(-

$\exists \circ-\operatorname{los} 2$

$x \rightarrow m \rightarrow \rightarrow \rightarrow t a$

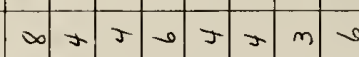

a

है के

틍

\begin{tabular}{c|c}
\hline E \\
\hline E \\
\hline
\end{tabular}

$\frac{1}{7}$
$\frac{1}{1}$
$\frac{1}{1}$
$\frac{5}{2}$
$\frac{0}{2}$

五

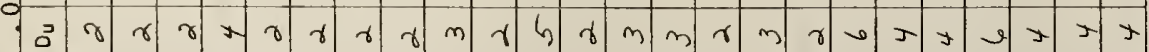

น.

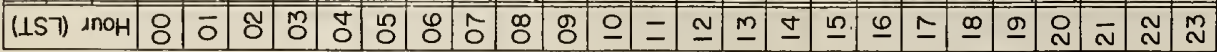

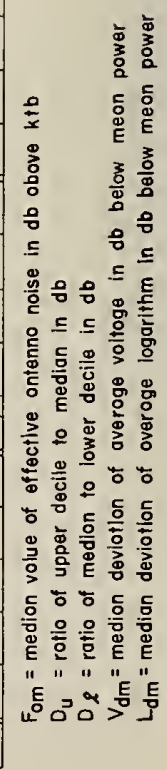




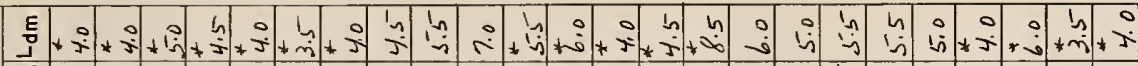

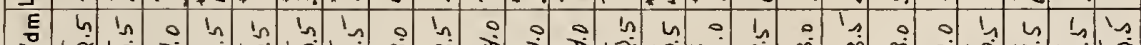

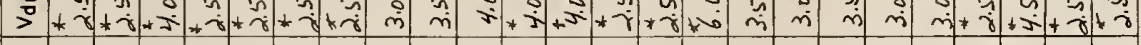

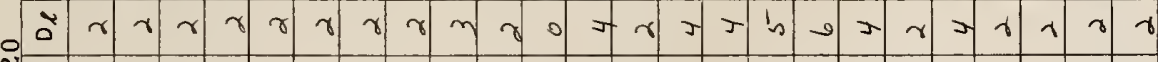

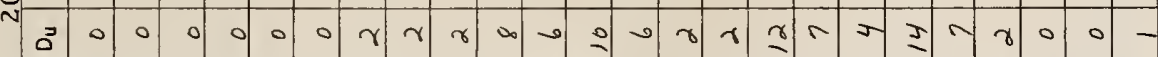
Е

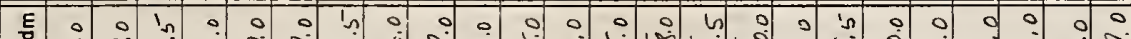

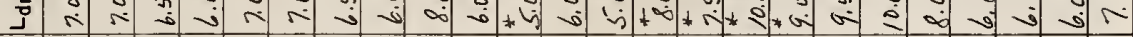
㞷

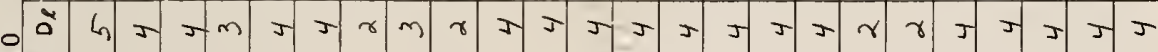

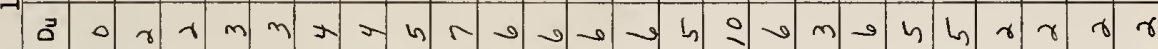

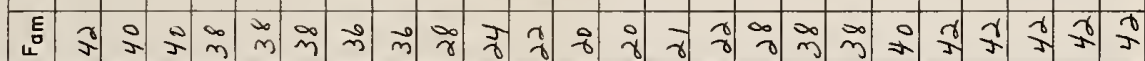

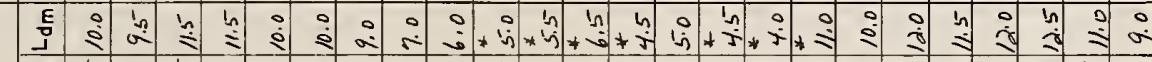
若

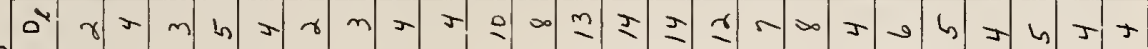

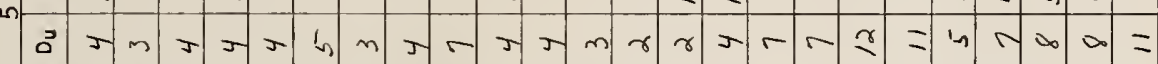
एँ

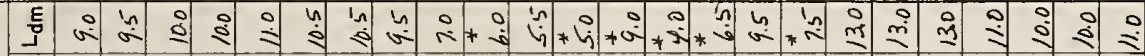

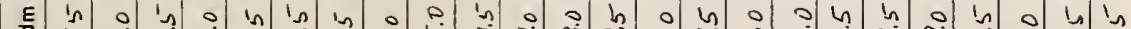

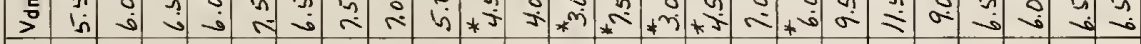

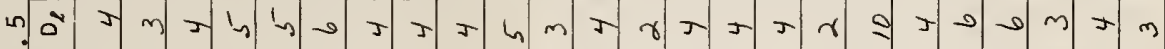
$\sum_{\underline{0}}$

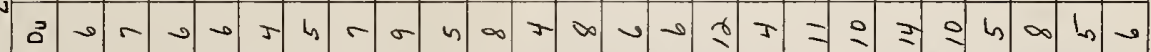
E $\rightarrow$ दा दर

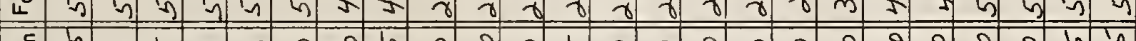

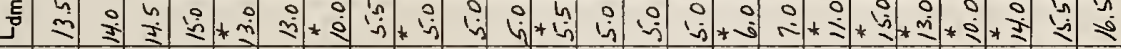

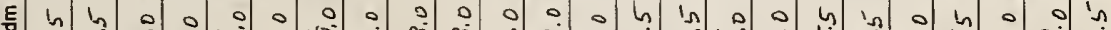
络文

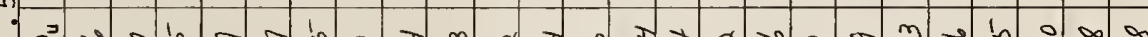

แลำ

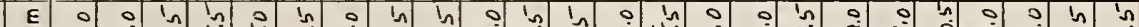

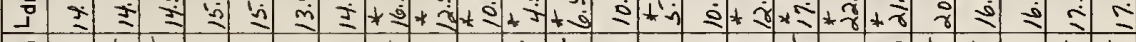

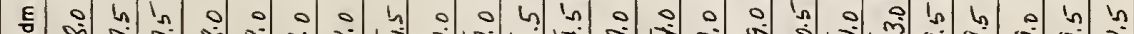

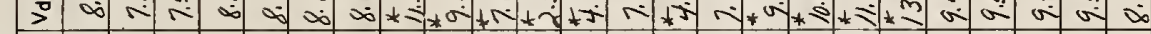

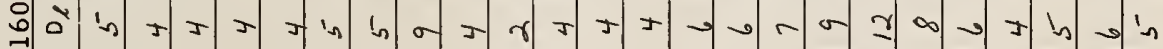

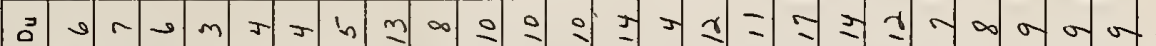
एँ ने के न :

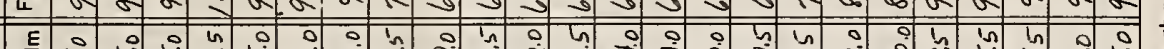

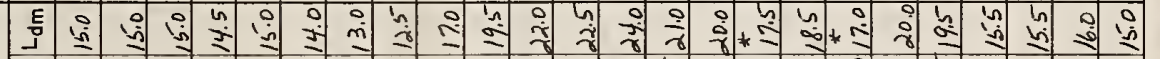

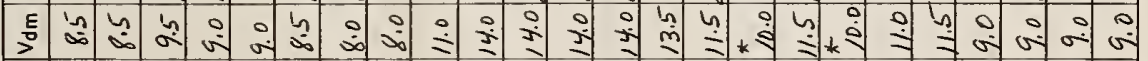
药 5. है

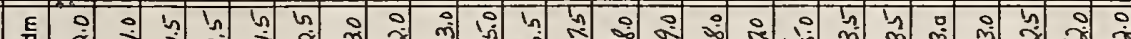
ग

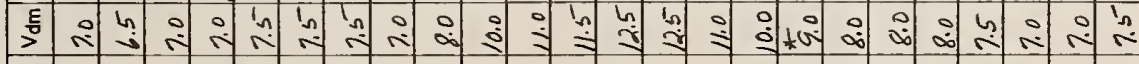

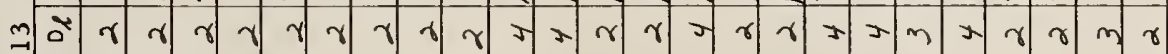
:

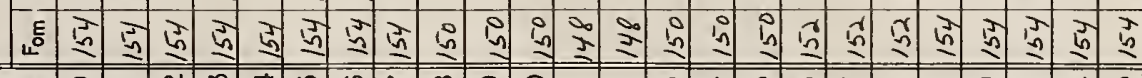

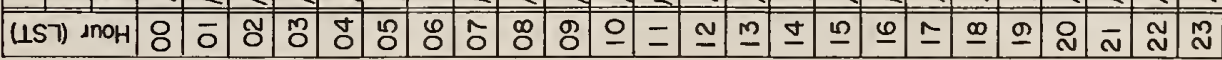




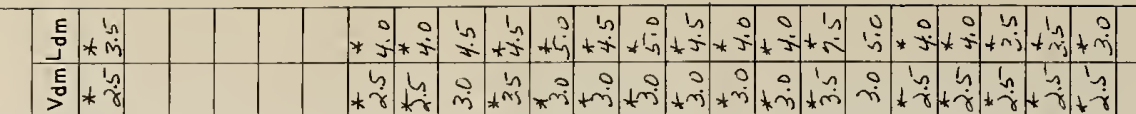

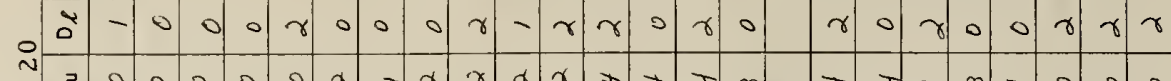

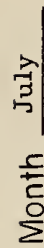

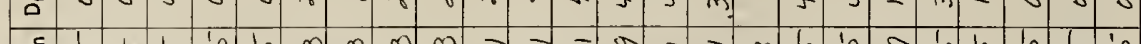

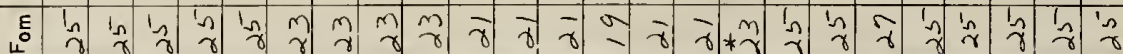

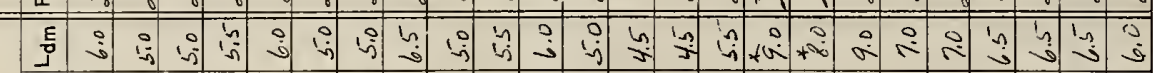

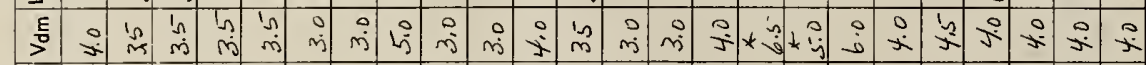

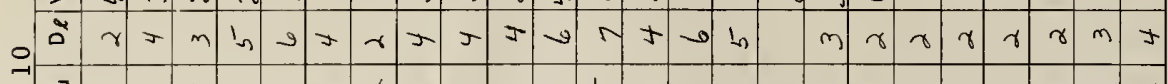

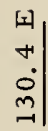

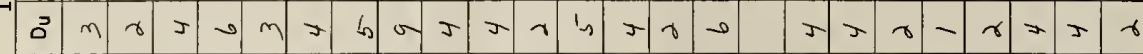

E

ह

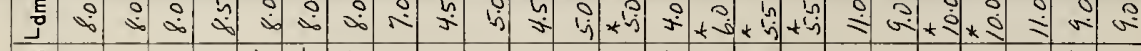

ธ்ุ

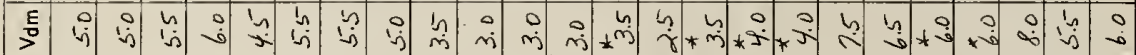

or r rmm m r r

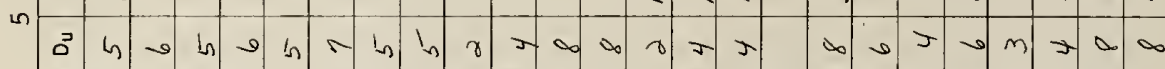

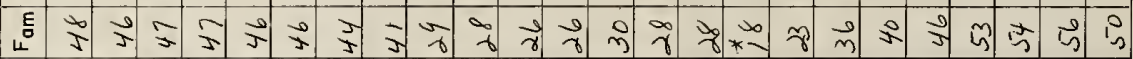

苛

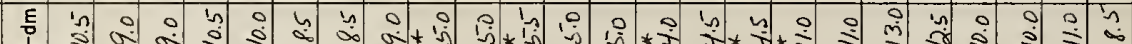

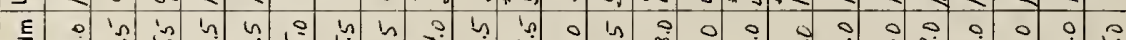

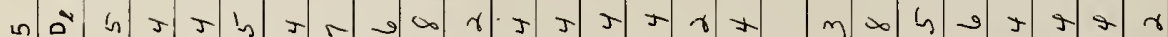

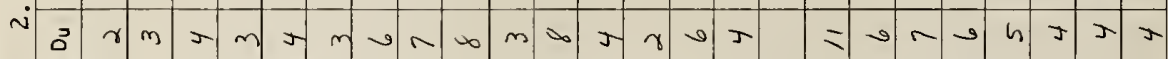

产

Uे $\quad$ ह

ब

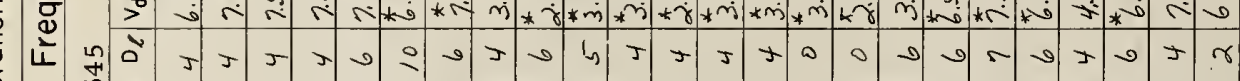

$\frac{1}{\infty}$

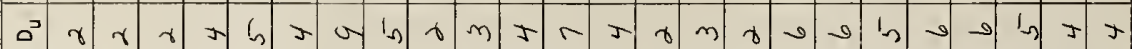

E

$\frac{w}{2}$

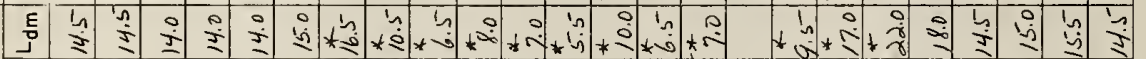

希

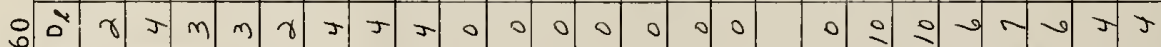

$\frac{0}{\frac{1}{0}}$

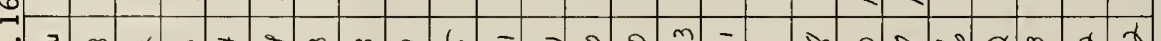

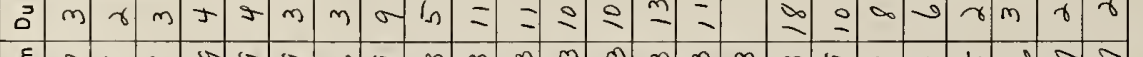

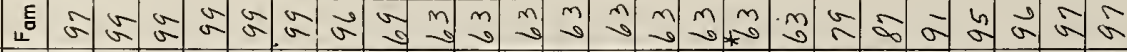

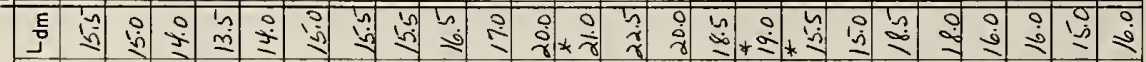

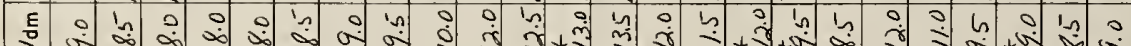

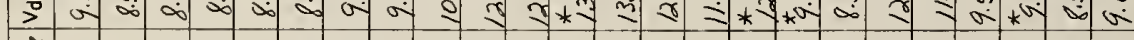

岂

ที

กิ-

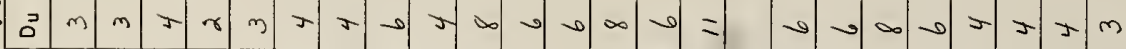

E

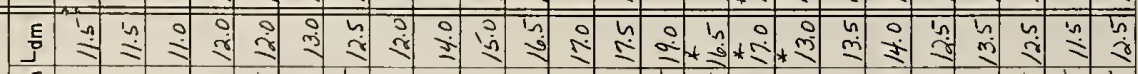

E

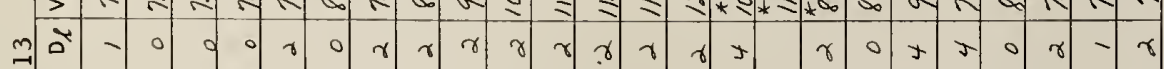

$\frac{1}{1}$

㐊

○े

-

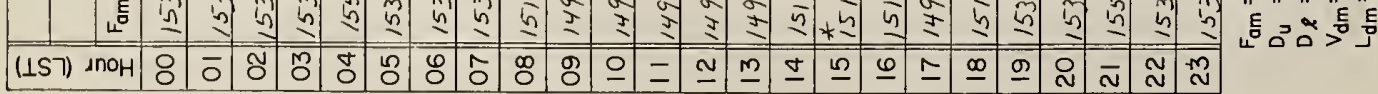




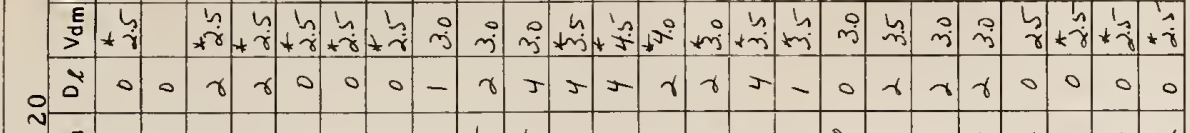
$\rightarrow$

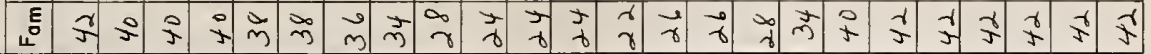

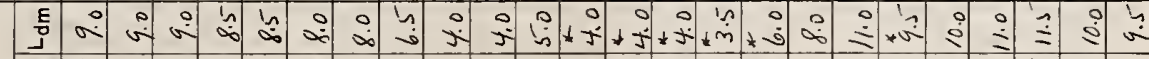

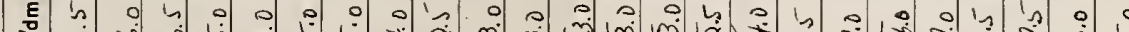

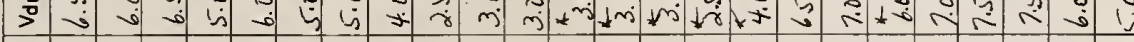

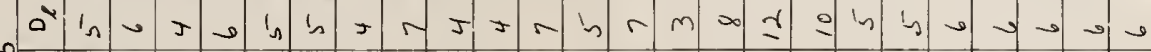

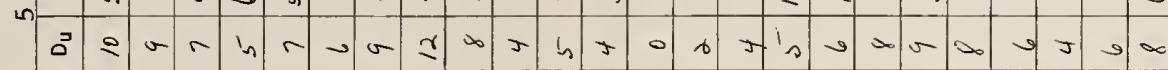
ह

9

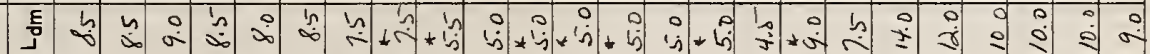

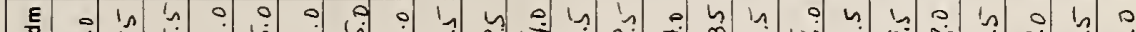

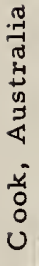

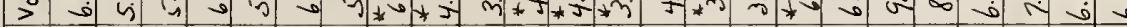

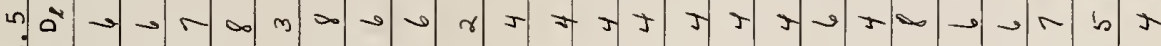

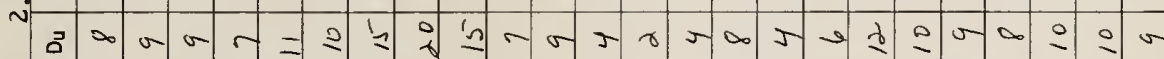

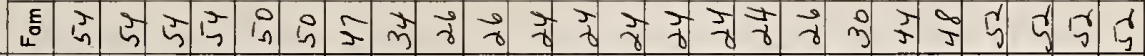

ह ह

E

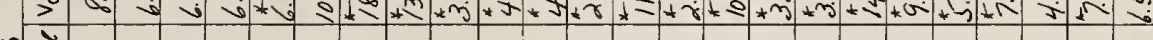

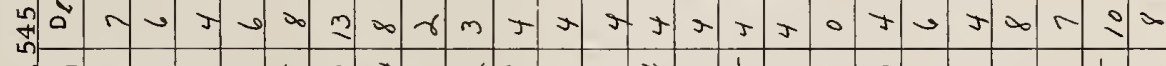

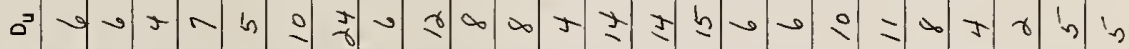

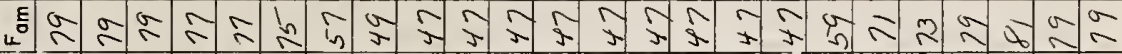

$\frac{w}{2}$

ह

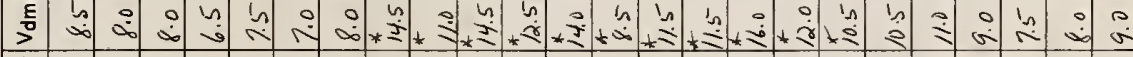

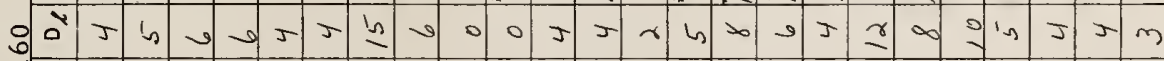

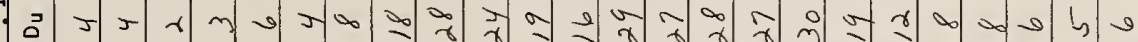

ह $:$ :

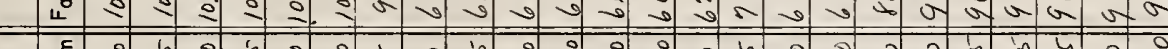




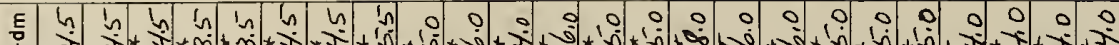

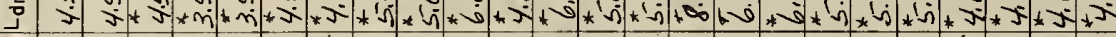

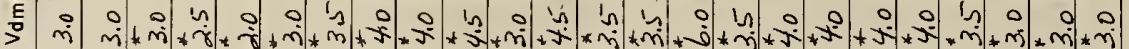

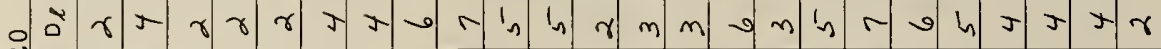

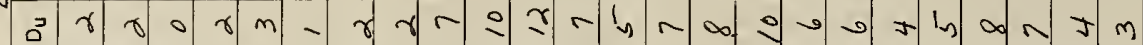

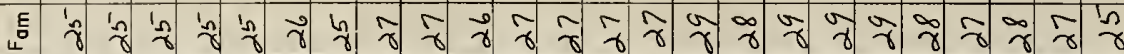

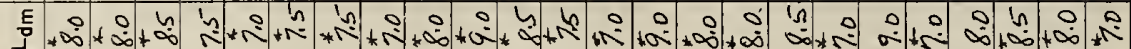

E 0 O O l u

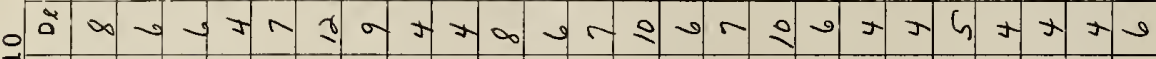

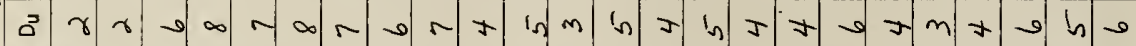

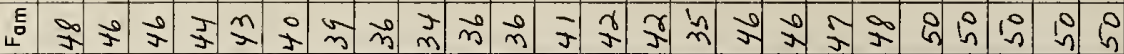

ஜ்

宁

z)

$\therefore$

일

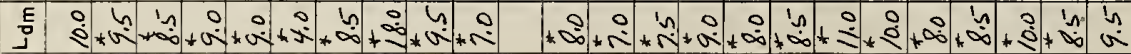

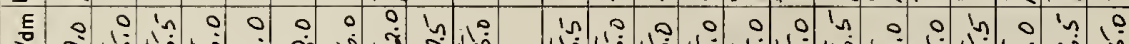

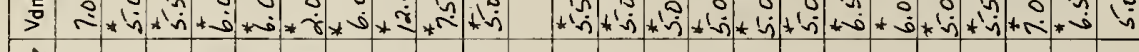

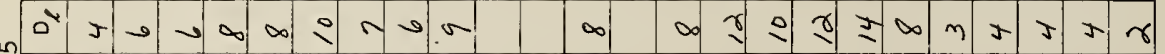

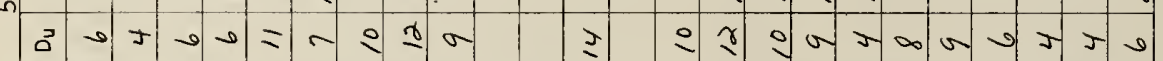

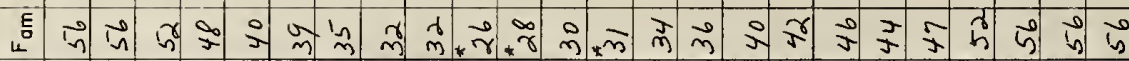

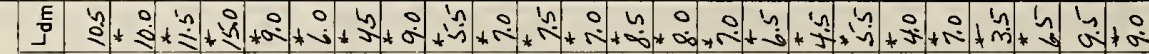
E

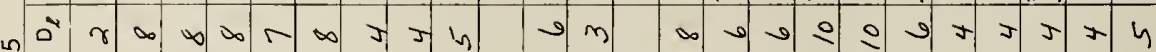

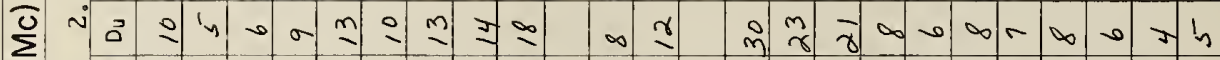

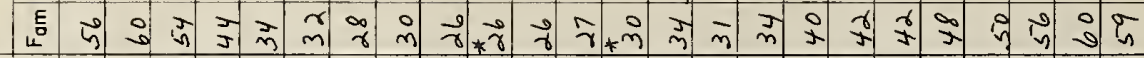
ठ

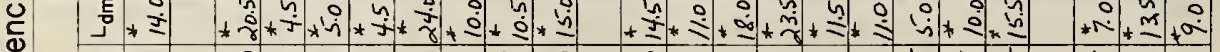

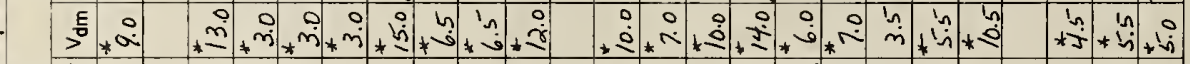

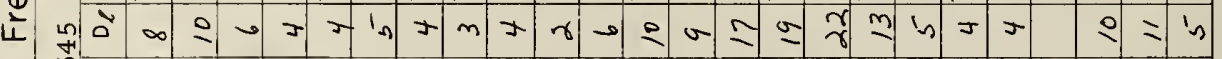

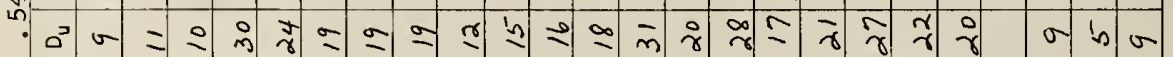

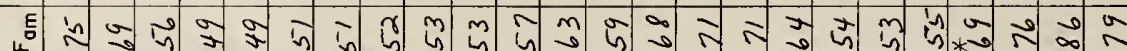

$\frac{w}{2}$ kE

*E

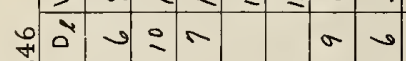

$\frac{0}{}$

ด 2 ช

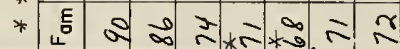

离

$$
\text { E }
$$

¿

ฉ

E

$\frac{1}{3}$
$\frac{1}{1}$
$\frac{1}{1}$
$\frac{1}{2}$

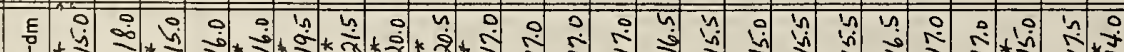

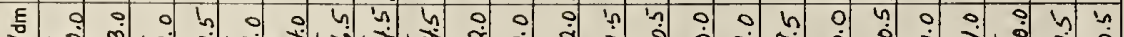
चี

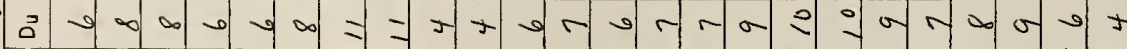

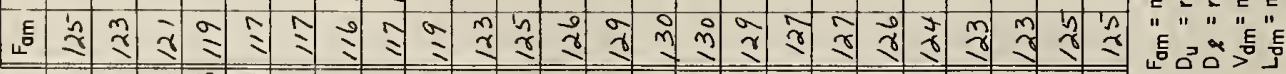
(157) גnoH 8 ठ 
E

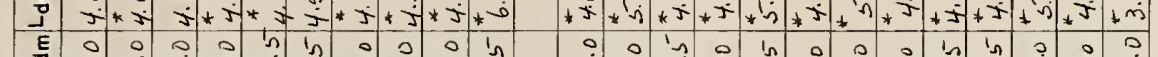

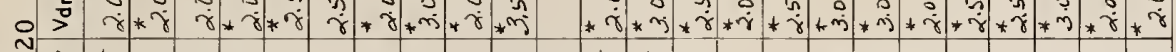

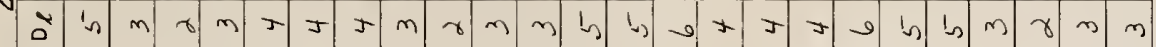

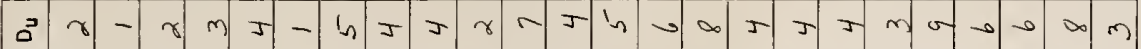

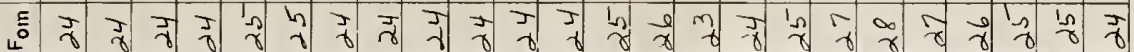

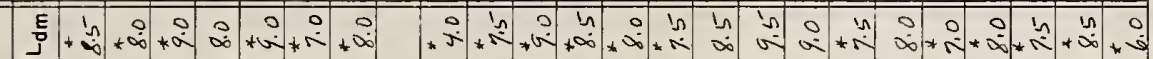

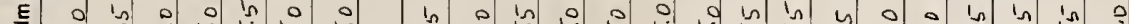

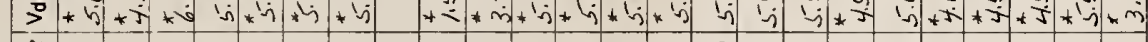
0 व

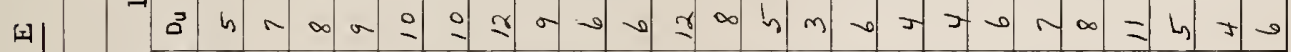
m. $\stackrel{-1}{-1}$

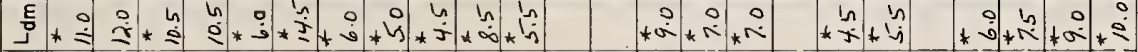

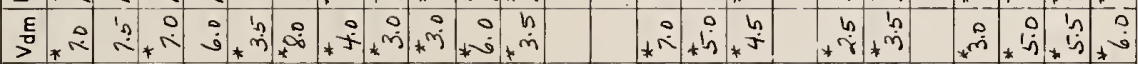

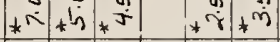

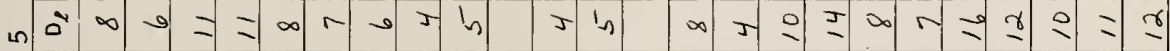

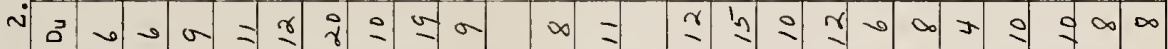

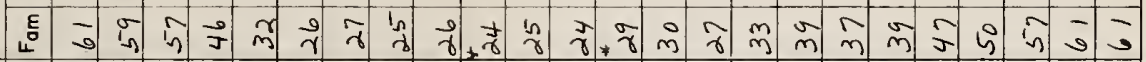

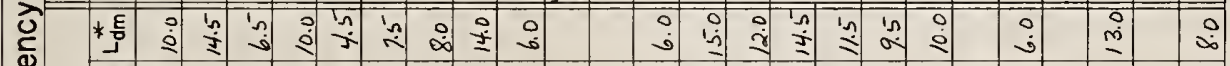

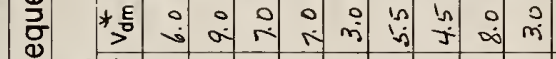

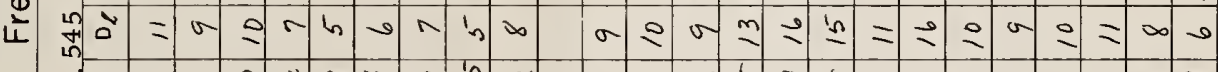

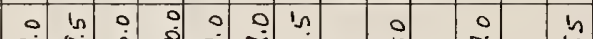

० न m $\gamma_{\gamma}^{\gamma}$ भै

Е

$\frac{w}{2}$

$+\frac{E}{\partial}$ 约

*E

ปั

울

* ว $\infty=$ ช

* हू भ व

4
0
4
5
5

군

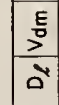

10

\begin{tabular}{c|c|}
\hline$\varepsilon$ \\
\hline${ }^{\circ}$ \\
\hline
\end{tabular}

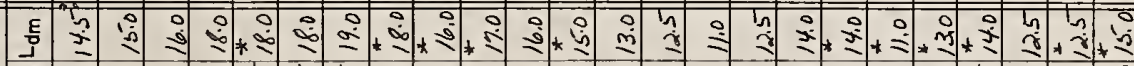

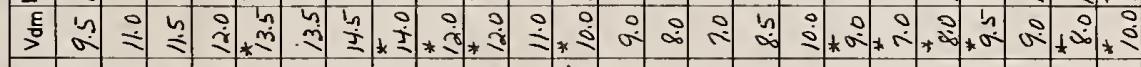

चี

몽
$\frac{1}{1}$
$\frac{1}{1}$
$\frac{2}{2}$

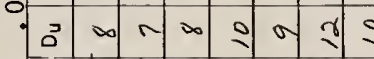

ผ

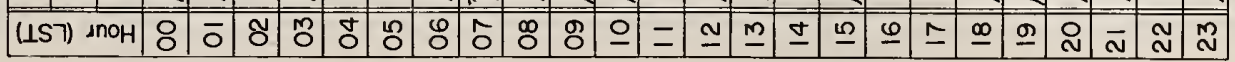

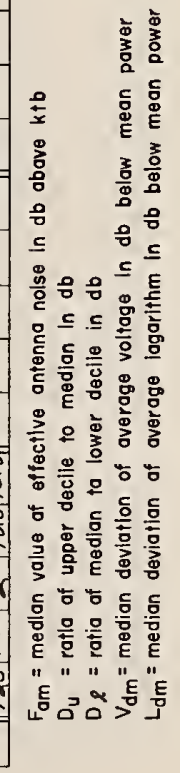


on

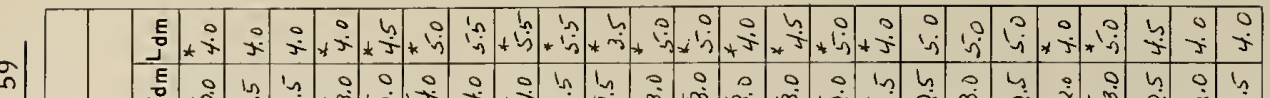

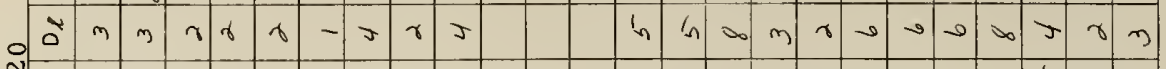

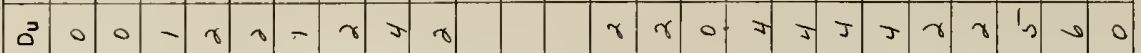

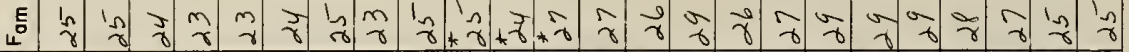

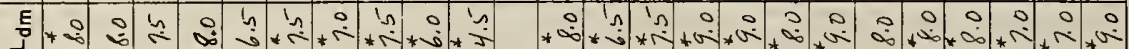

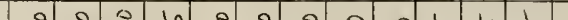

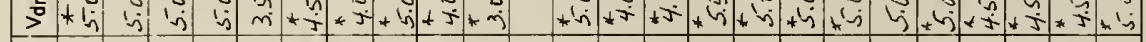

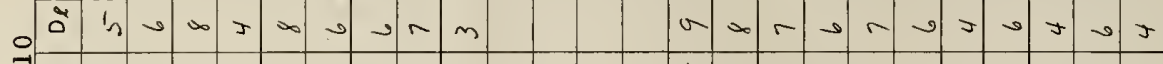

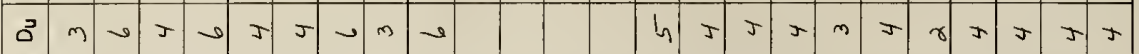

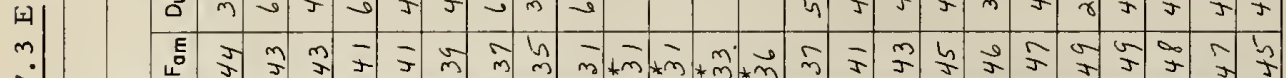

$\stackrel{m}{\sim}$

ণ্்

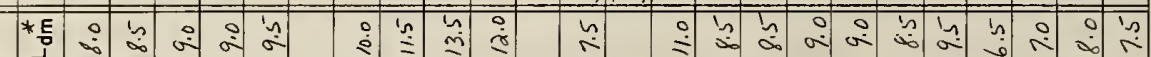

\#

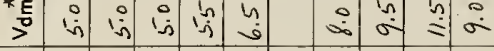

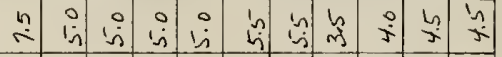

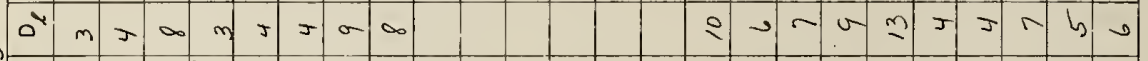

$Z$
Zn
$\dot{0}$
in

$+\check{j}$

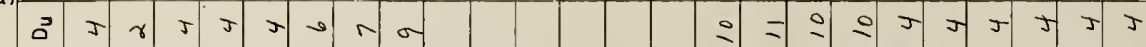

E

E

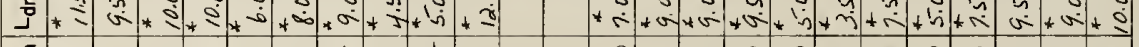

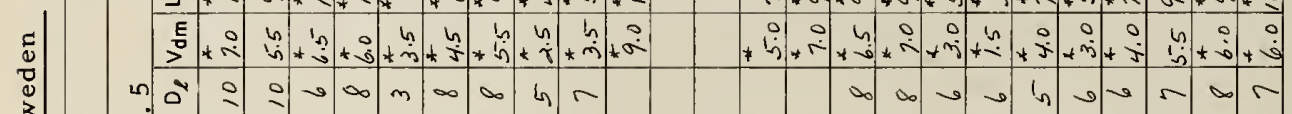

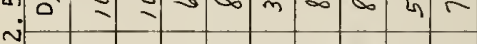

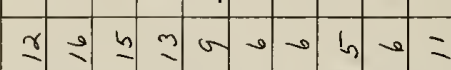

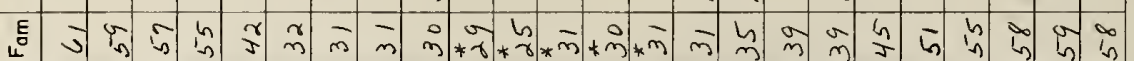

$\mid \begin{gathered}\frac{2}{0} \\ \frac{c}{0} \\ \frac{0}{5} \\ \frac{0}{2} \\ \frac{1}{4}\end{gathered}$

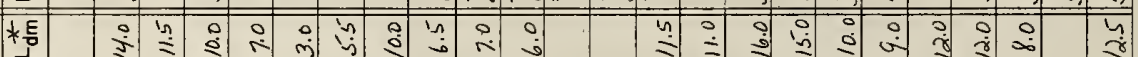

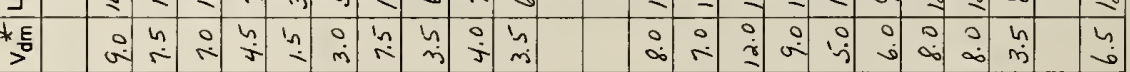

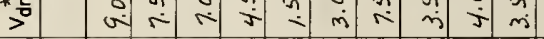

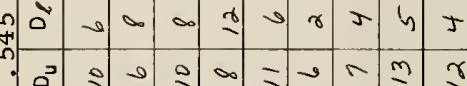

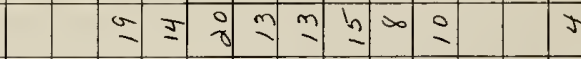

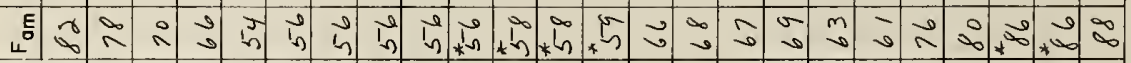

$\frac{W}{2}$

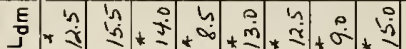

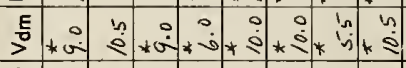

ț

$\frac{0}{2}$

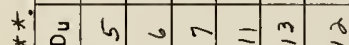

$\underset{1}{\alpha}$

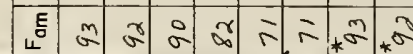

虫

s

崫

ह

¿

3

点

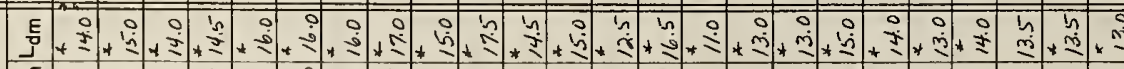

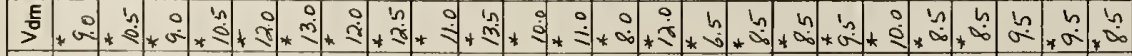

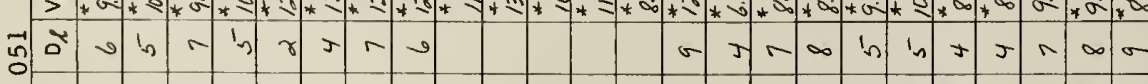

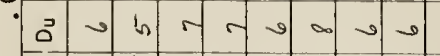




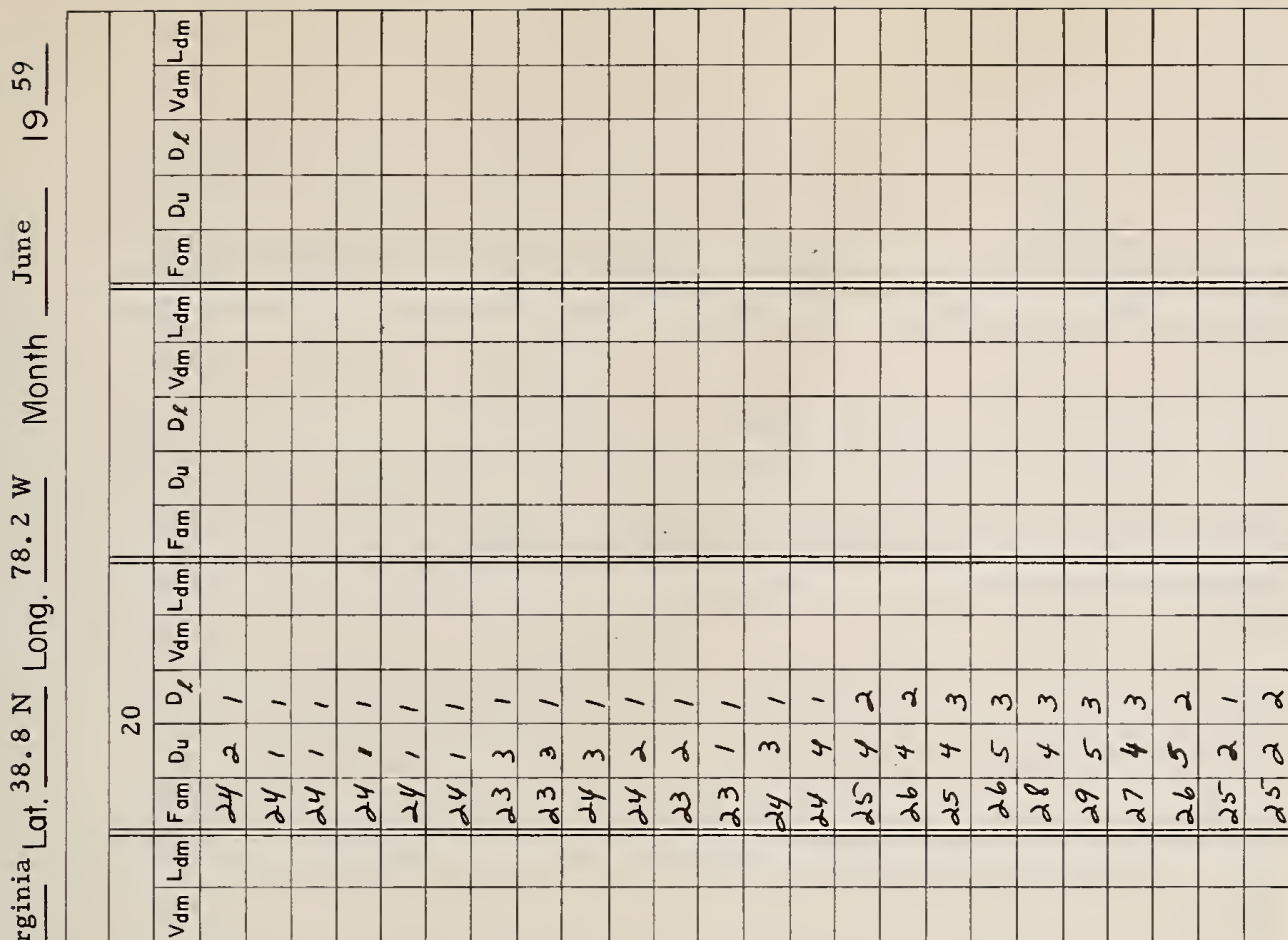

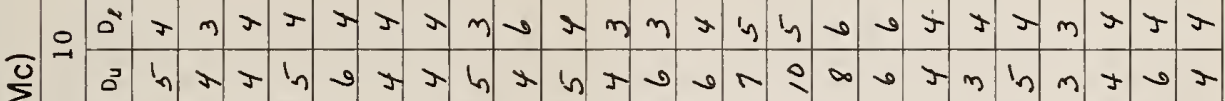
ह त 4

E

$\frac{E}{>}$

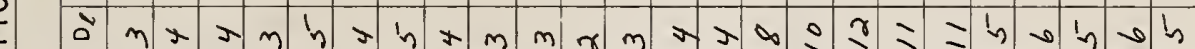

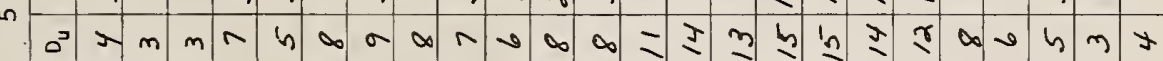

น

$\frac{w}{2}$

E

통

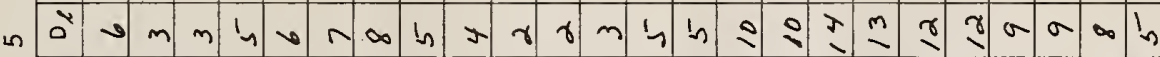

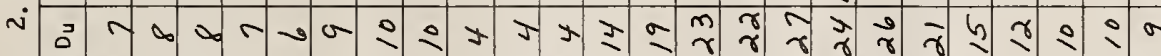

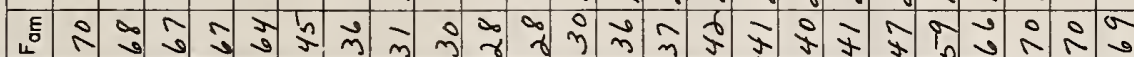

E्ञ

통

岂

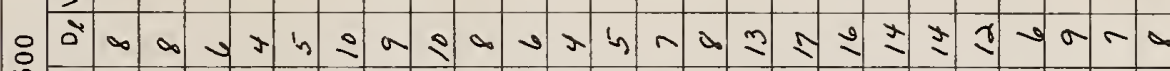

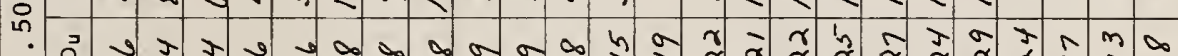

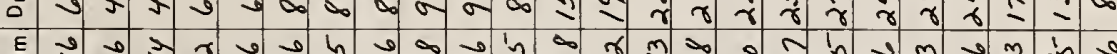

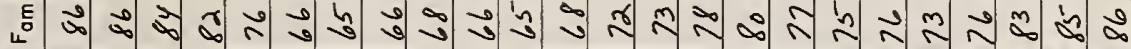

经

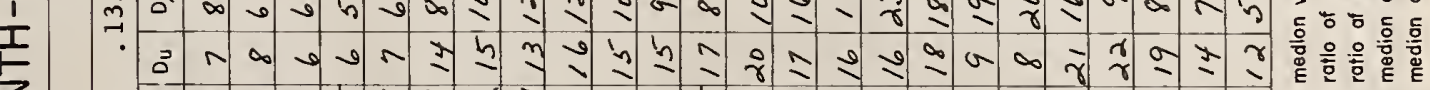
น

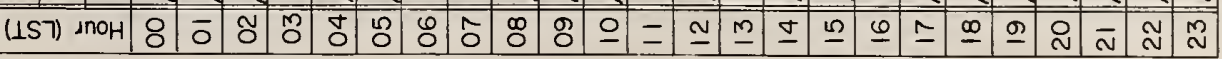


$0^{3}$

L

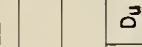

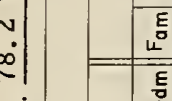

它

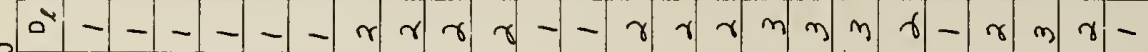

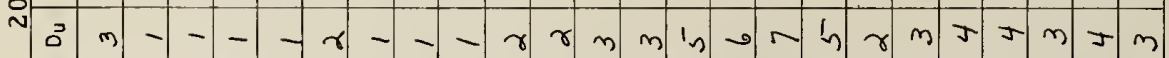

E

E⿱丷⿹弔㇒

퉁

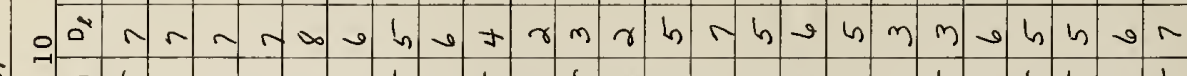

¿ 0 a

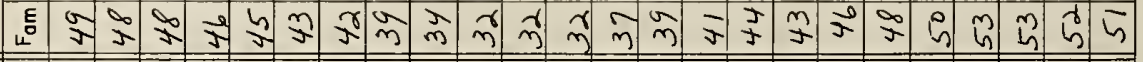

তे

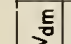

可

Lั

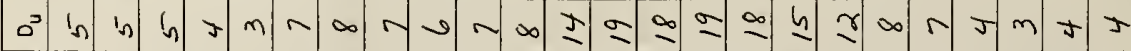

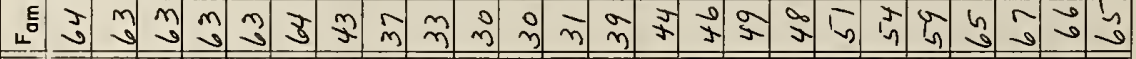

$\frac{w}{2}$

$\stackrel{E}{9}$

E

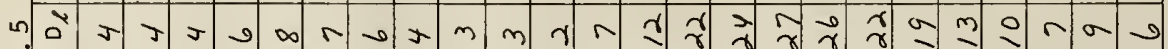

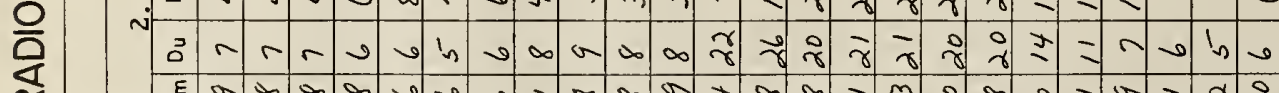

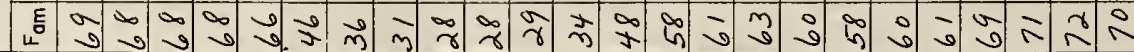

㟶

E

w

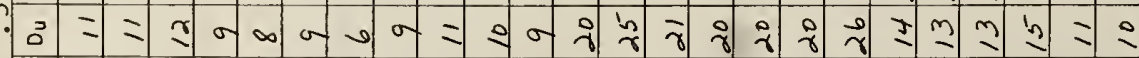

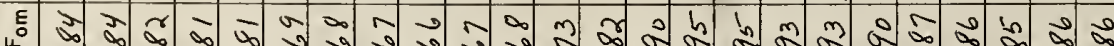

$$
\text { Eᄐ }
$$

E

है

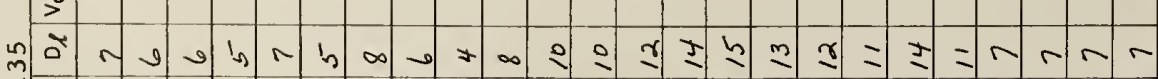

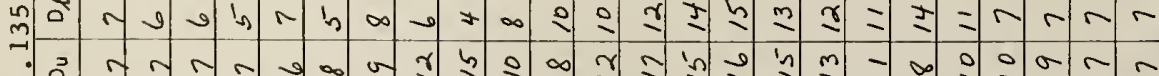

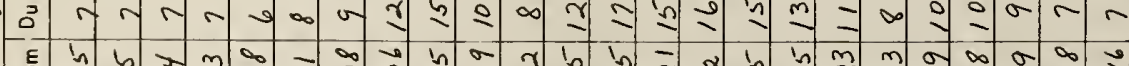

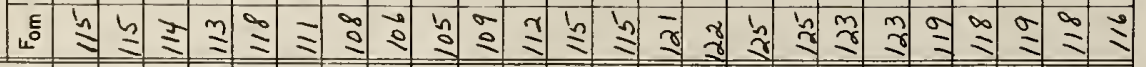
(1s7) 1 1กH 
in

a

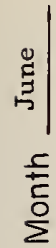

is

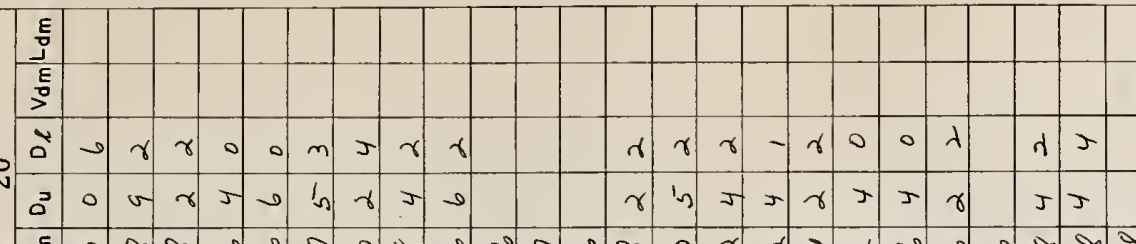

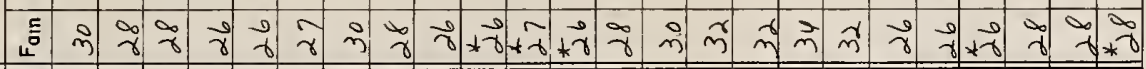

है

$\stackrel{5}{>}$

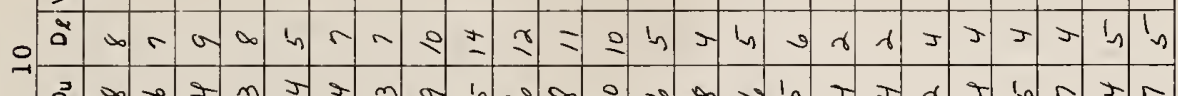

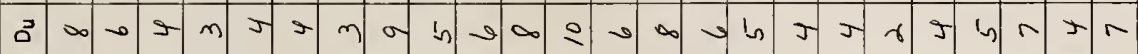

ब이

ह.

皇

ஜृं

ह5:

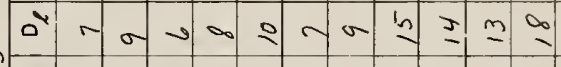

z

$\stackrel{4}{\sim}$

苛

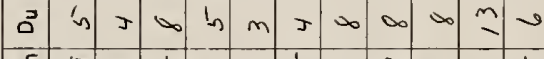

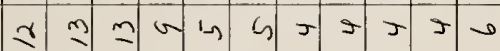

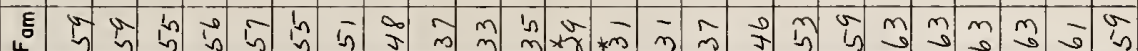

$\frac{E}{\square}$



$\frac{E}{2}$

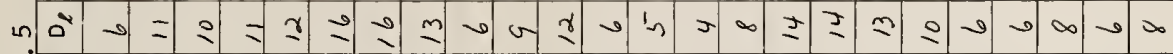

ग

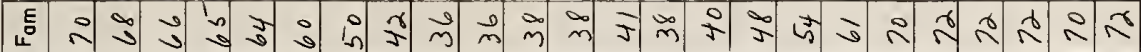

ते

总

E

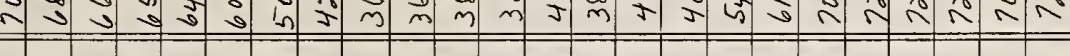

E

in

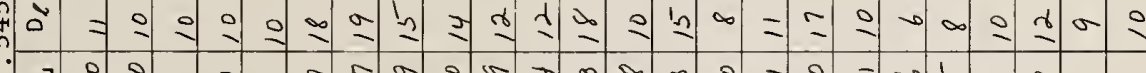

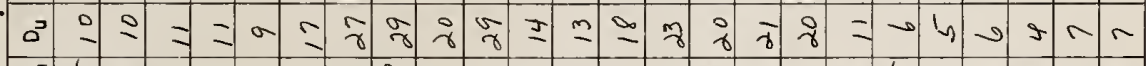

น

$\frac{\mathrm{m}}{\mathrm{O}}$

흠

\begin{tabular}{|l|l|l}
\hline 5 \\
\hline
\end{tabular}

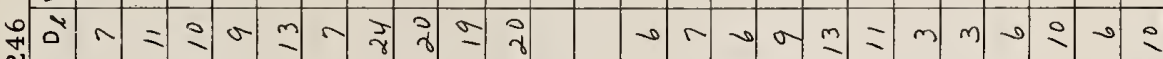

음

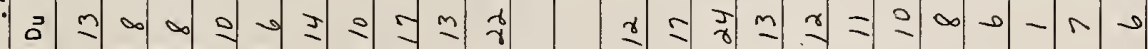

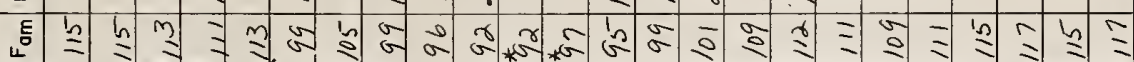

है

ह

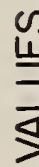

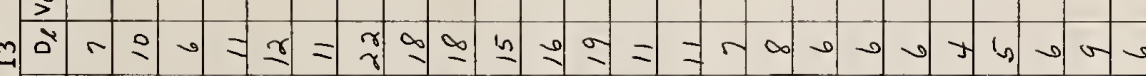

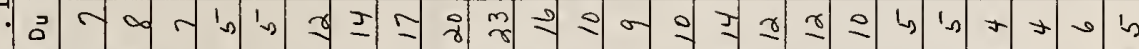

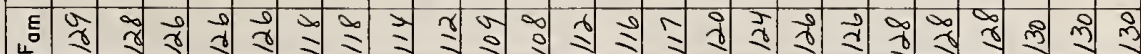

空

$\frac{\text { 똑 }}{\frac{0}{1}}$

崖

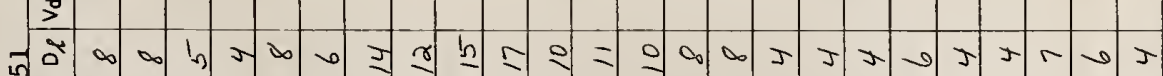

ñ $\infty$ b $\infty$ क

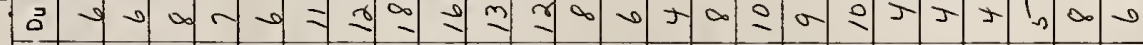

ผ

(1S7) यกㄱ 


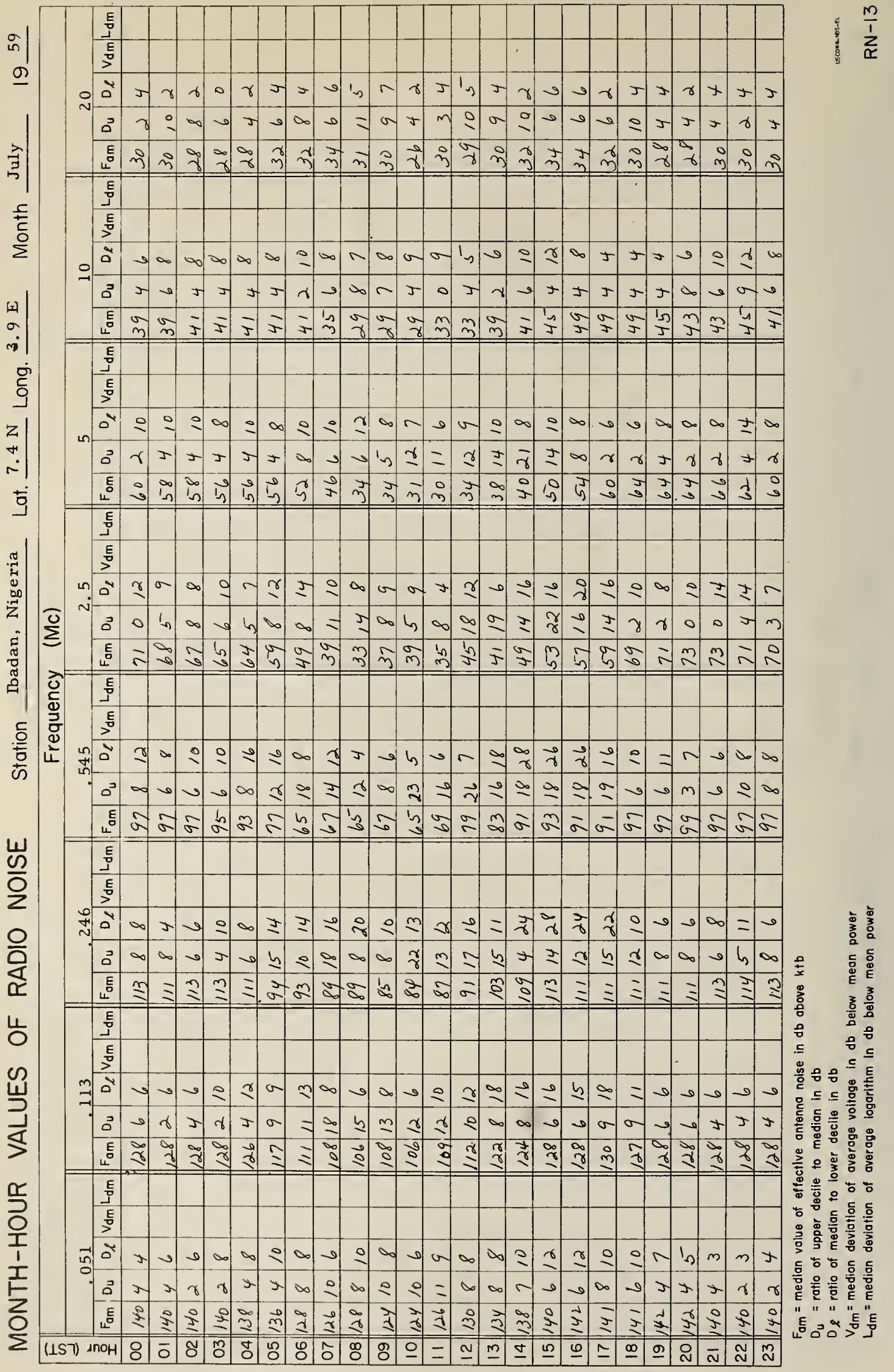


in

$\underline{\sigma}$

苟

달

3

్ㅗㅇ

z

N

ज्ञ

落|

등 공

훈

ভ̃

in

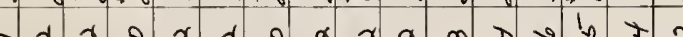

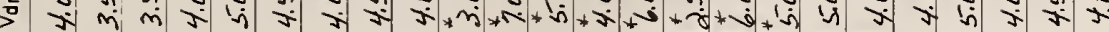

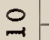

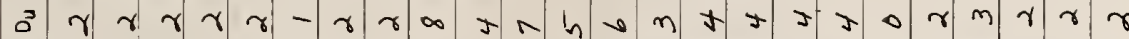

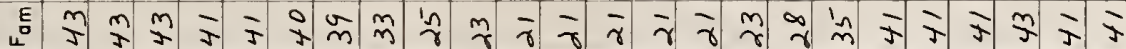

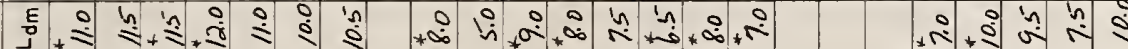

E 0 in in in 0 n 0 in 0 in in 0

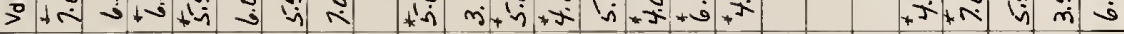

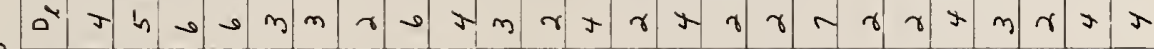

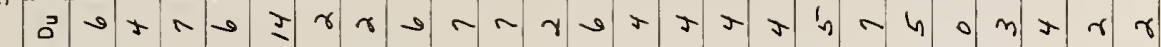

E तิ

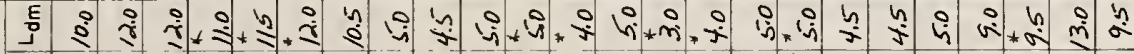

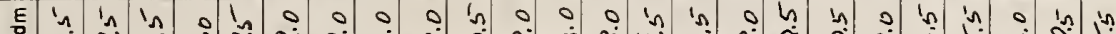

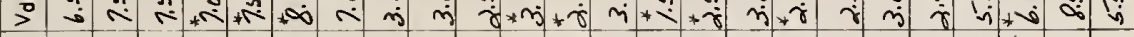

and

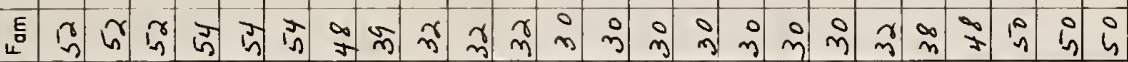

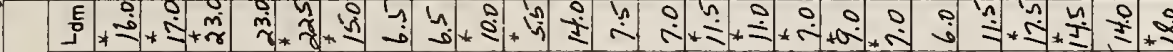

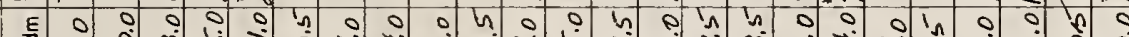

$>0$

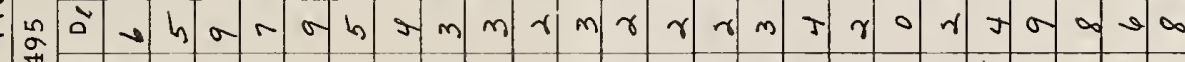

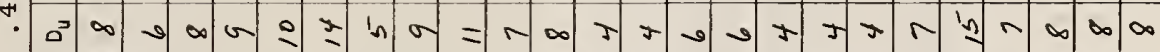

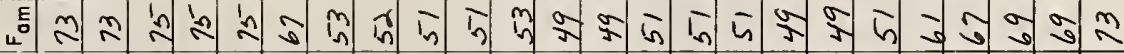

$\frac{w}{2}$

은

$\frac{1}{1}$

岁

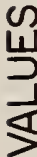

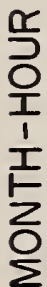

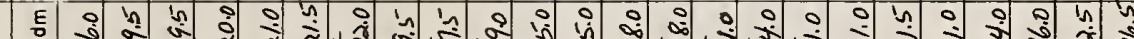

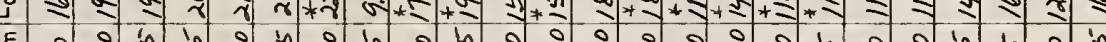

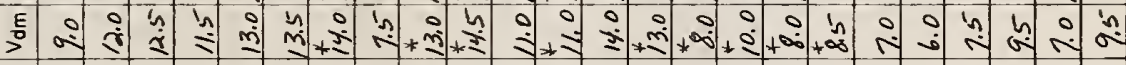

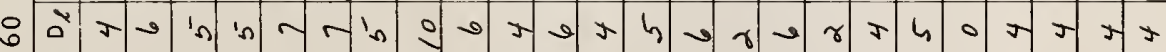
$\because$

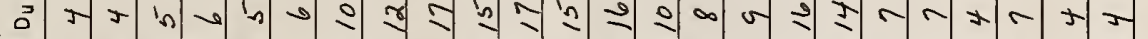

ह

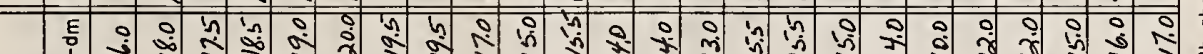

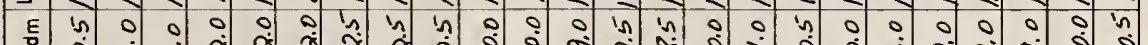
>

चี -

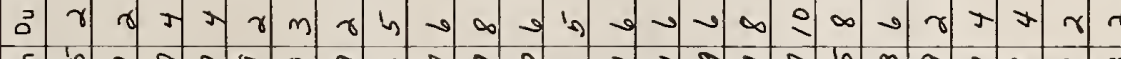

E

$E$ O

न $\frac{1}{5}$ ₹ 若 $\underset{0}{a}-\gamma \gamma \gamma \gamma-\gamma \gamma \gamma-\gamma-\gamma-\gamma \gamma \gamma-\gamma(\gamma) \gamma \gamma \gamma$ .

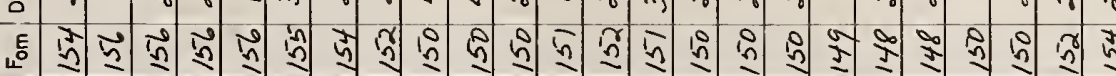

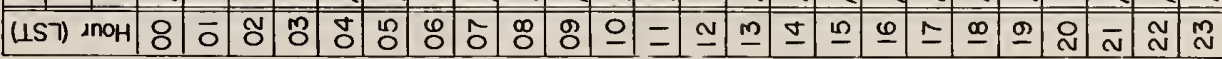




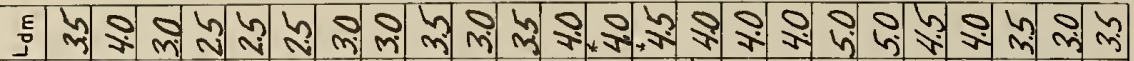

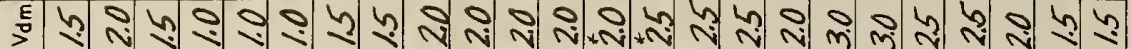

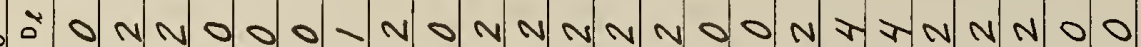
J N N m m MN N N N N N M N N N N N

E

ร

E 웅

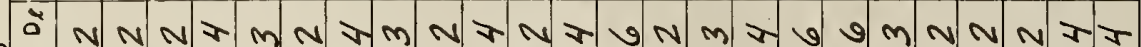

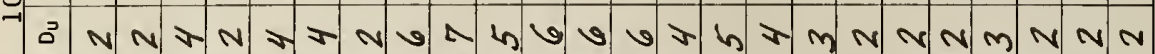

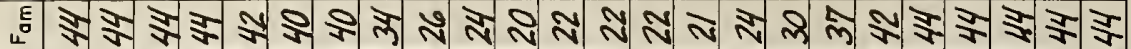

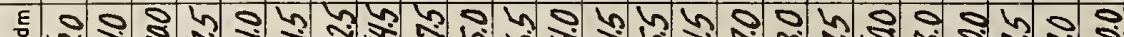

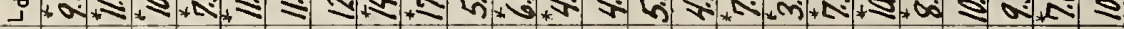

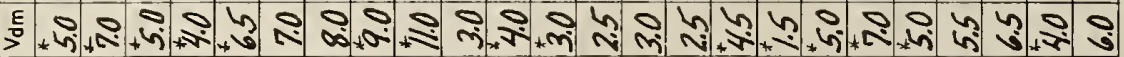

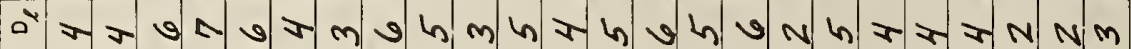

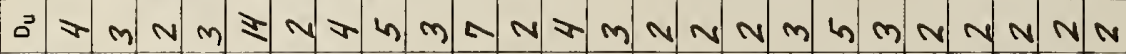

है फु

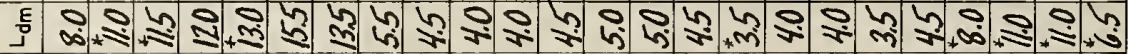

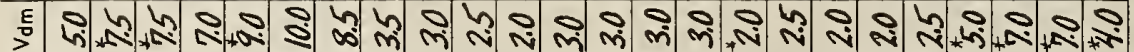

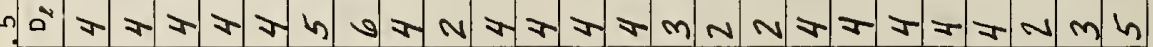

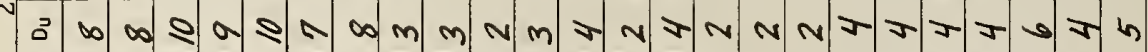

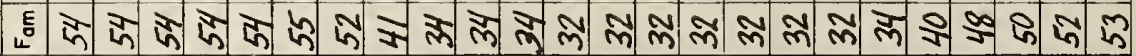

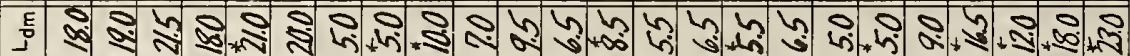

ह

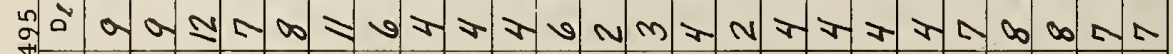

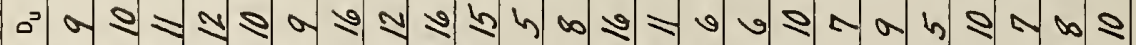

ह

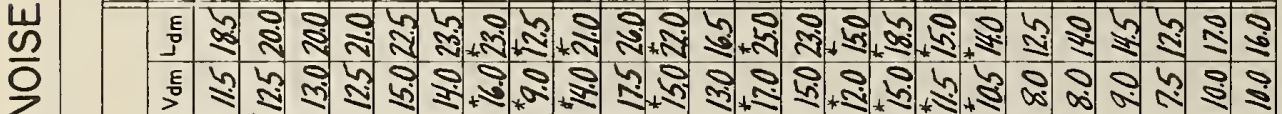

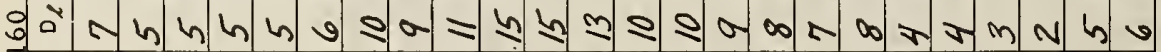

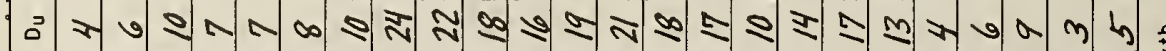

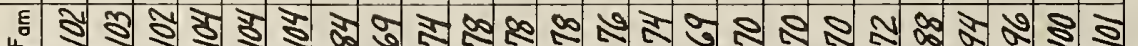

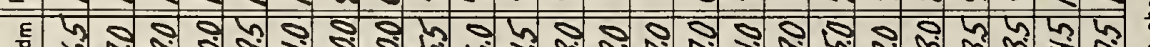

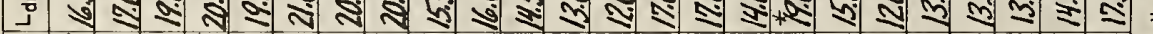
틍 원

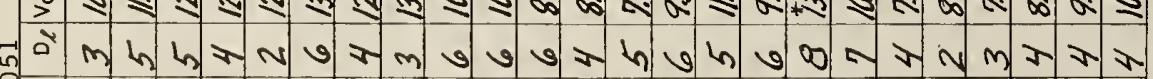

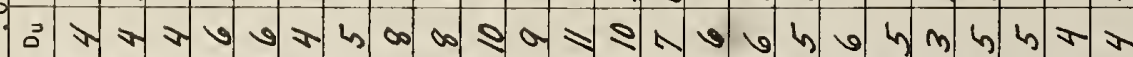

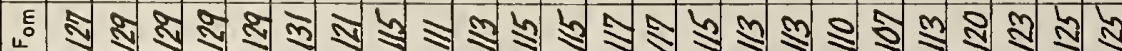

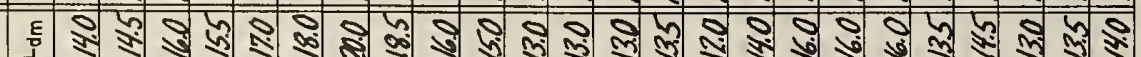


‡. E $h$ b

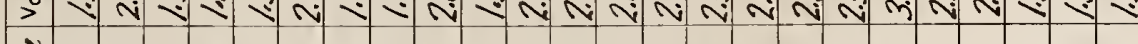

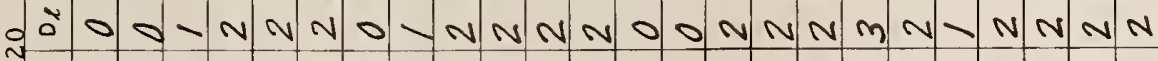

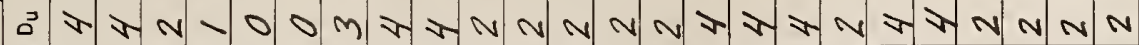

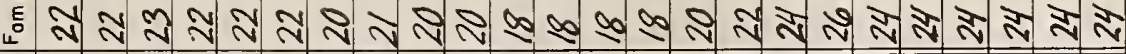

ह

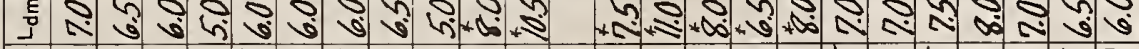

ह 웅

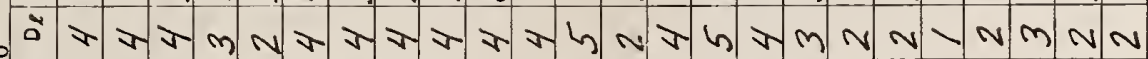

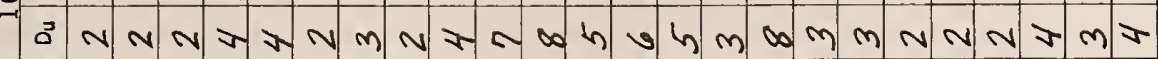

3

$\sim$

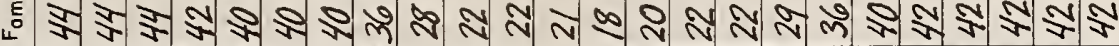

$\stackrel{2}{\rightarrow+1}$

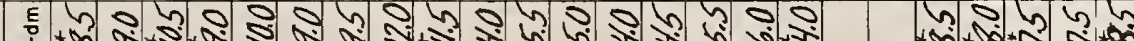

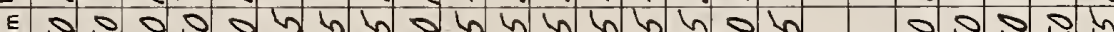

> +

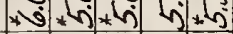

z

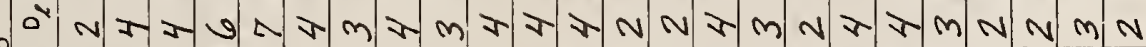

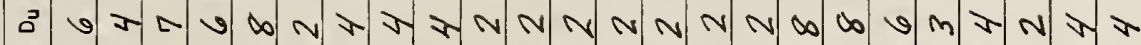

ह

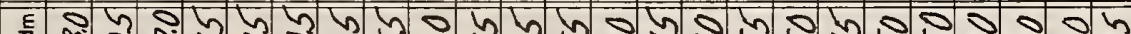

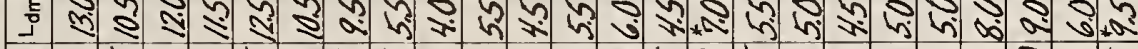

ह :

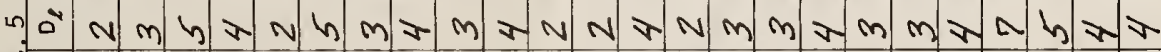

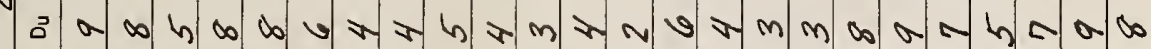

है

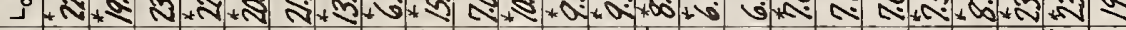

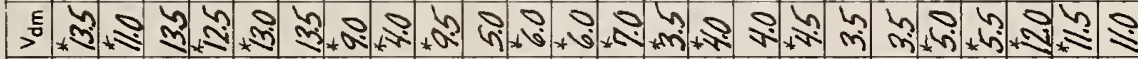

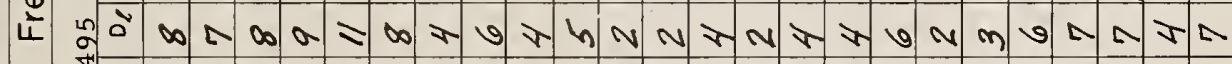

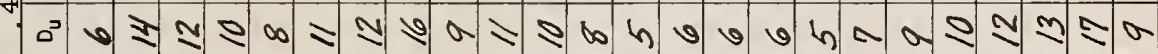

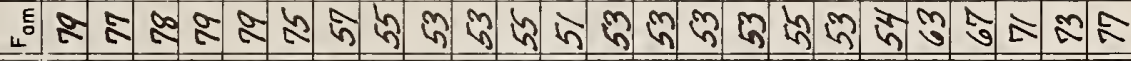

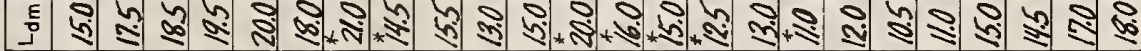

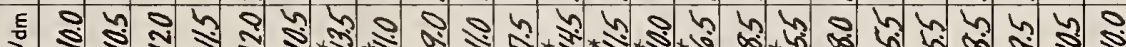
o

음

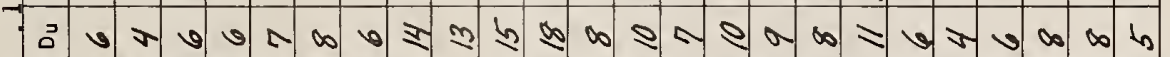

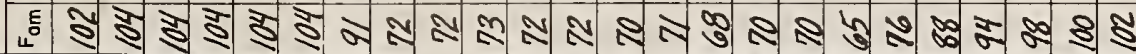

岁

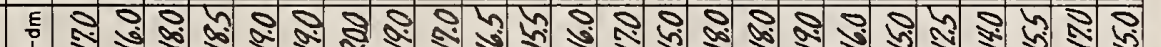

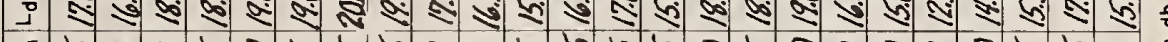

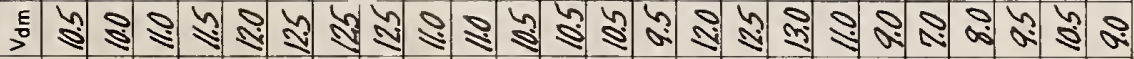

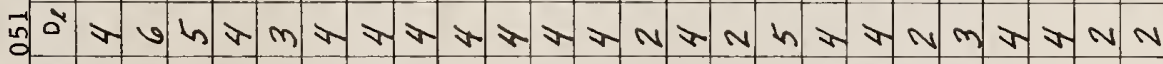

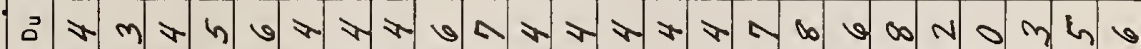

年

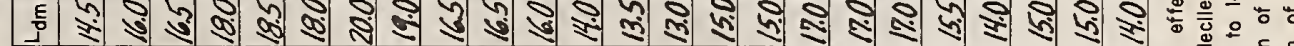

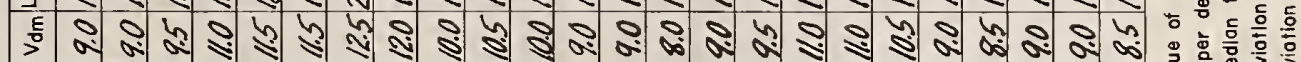

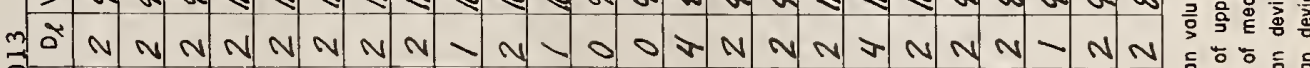

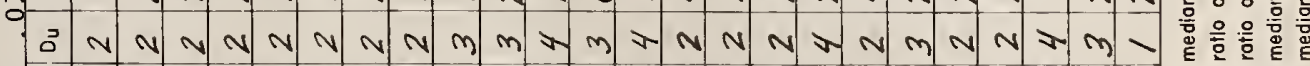

它

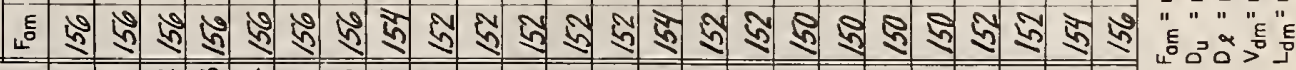

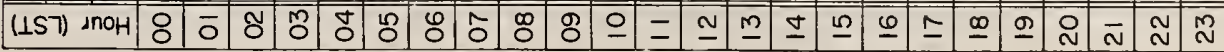


E म

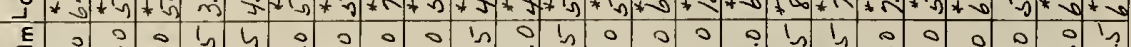

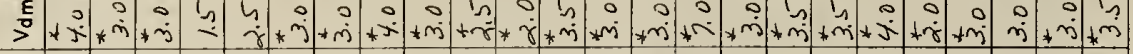

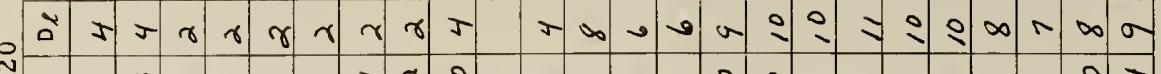

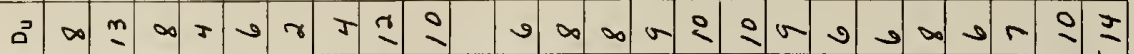

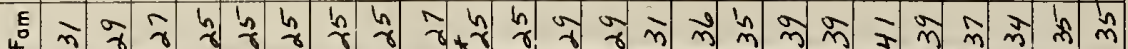

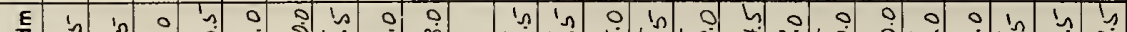

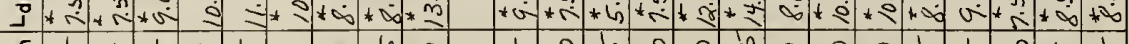

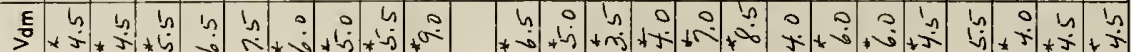

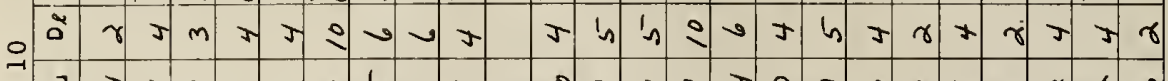

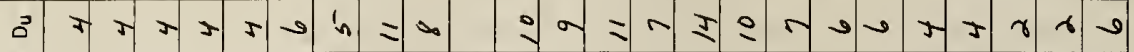
되. E 4
0.0
0

$z_{1}$

ind

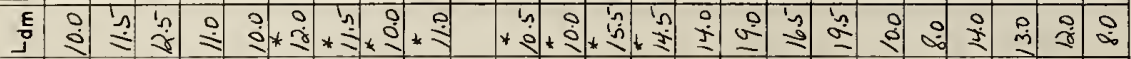

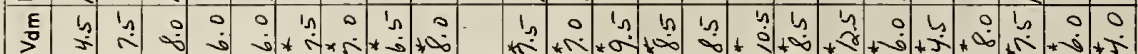

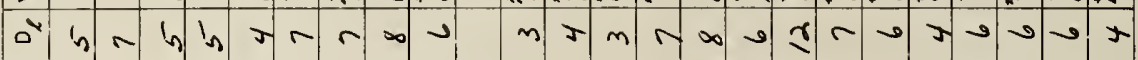

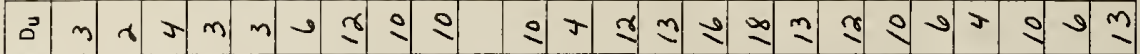
है

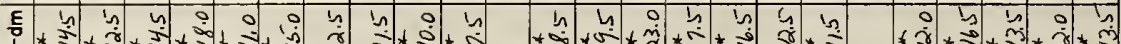

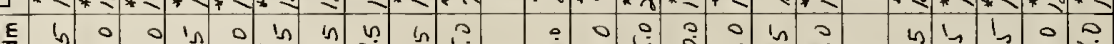

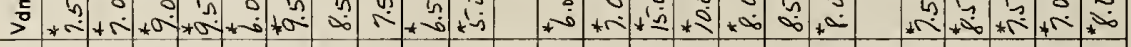

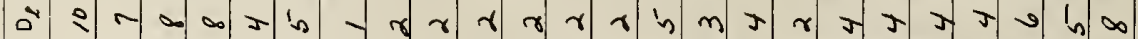

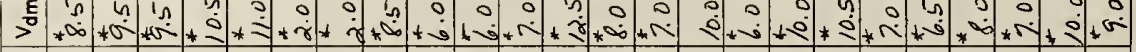

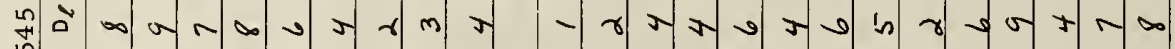

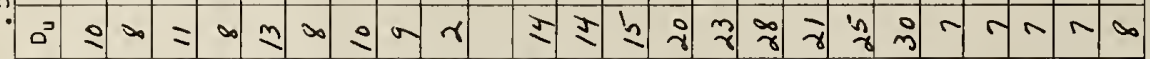

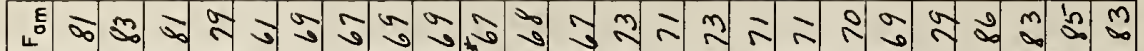

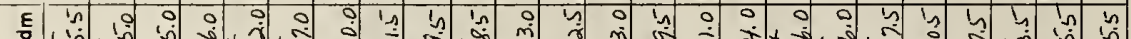

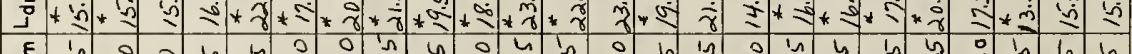

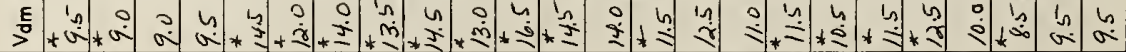
: व

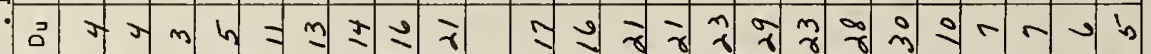

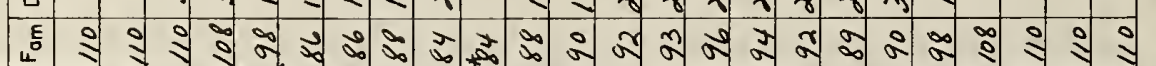

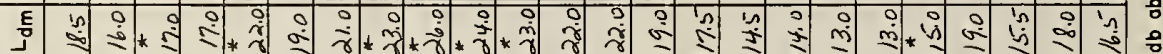

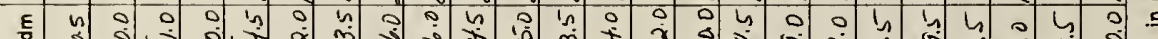

荘

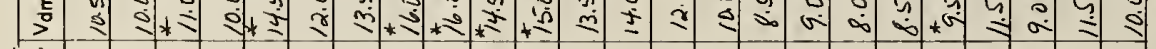

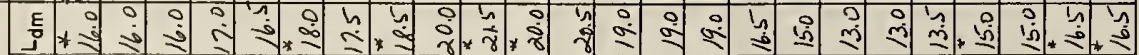

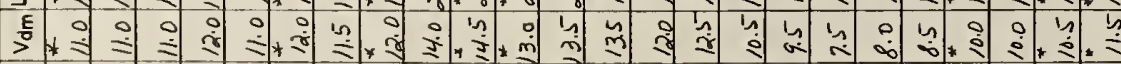

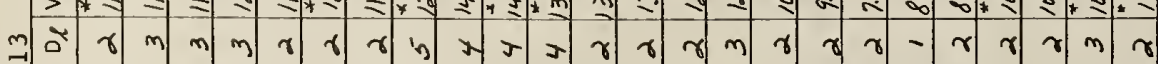

은 

is

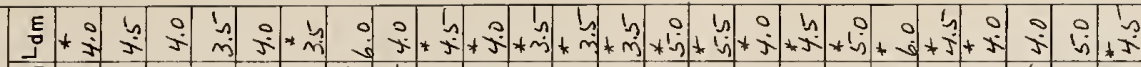

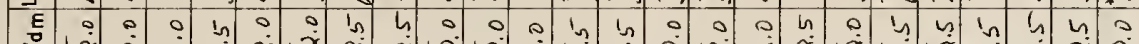

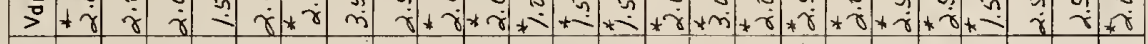

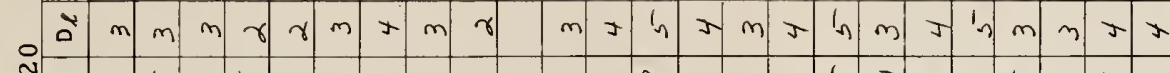

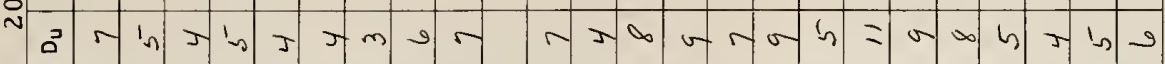
Бे

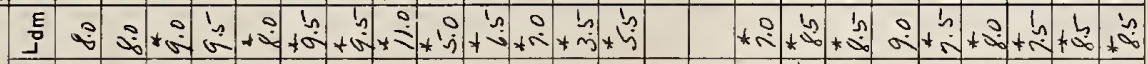

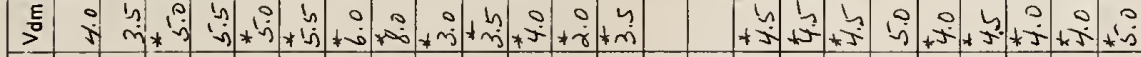

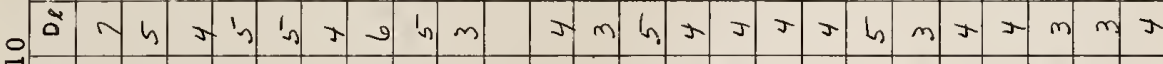

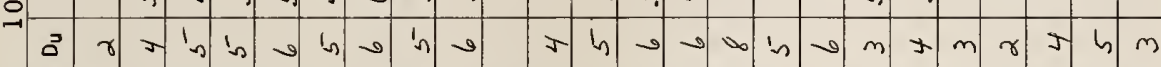

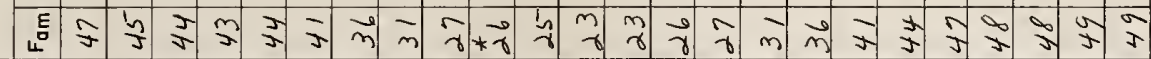

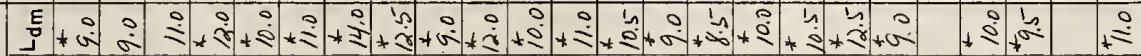

E 0 : 0 O

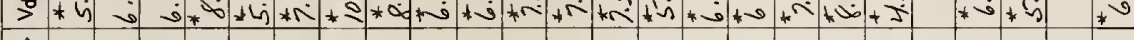

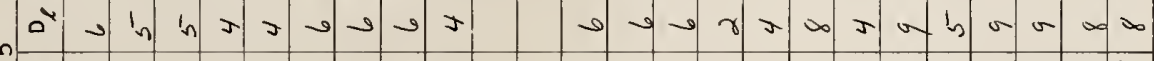

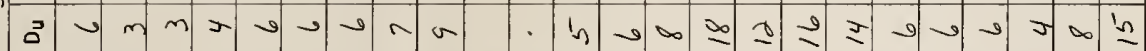

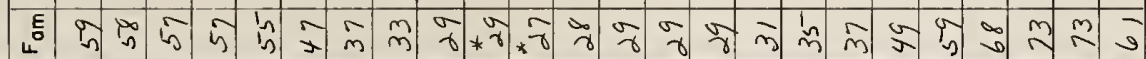

E

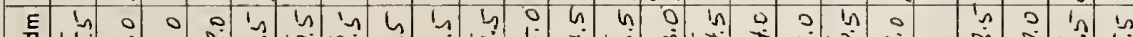

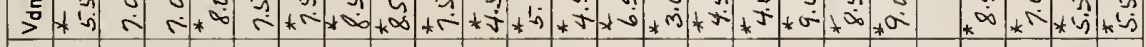

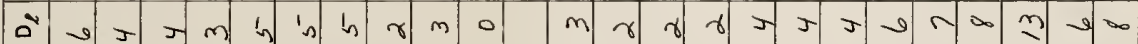

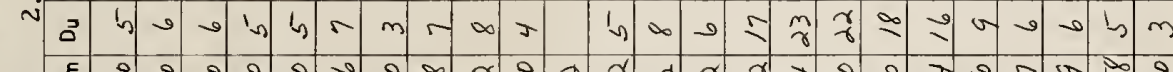
E

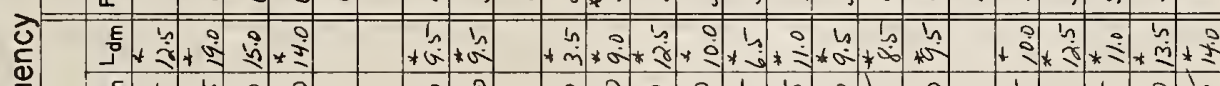

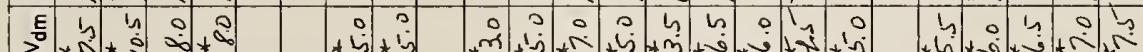

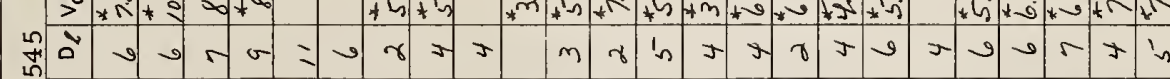

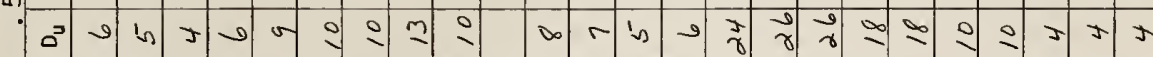

एह

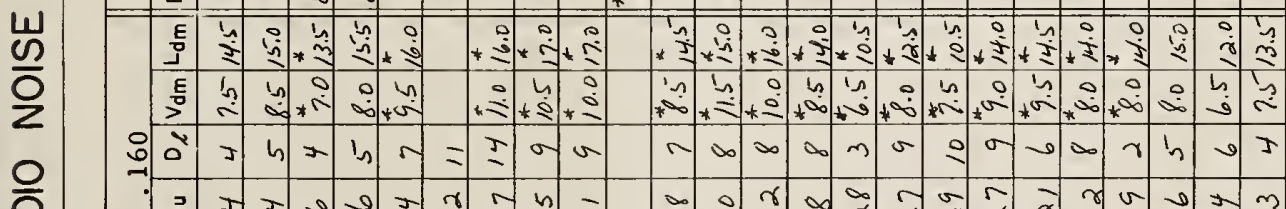

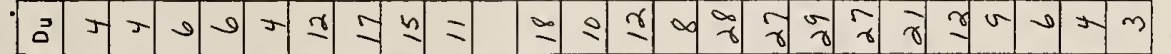
นE E के

-5
-2

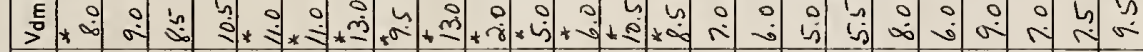

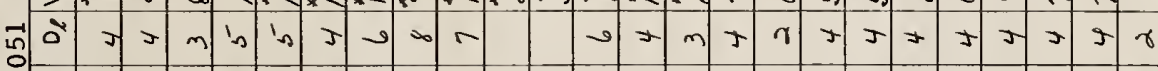

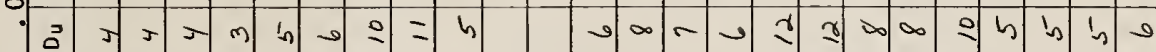

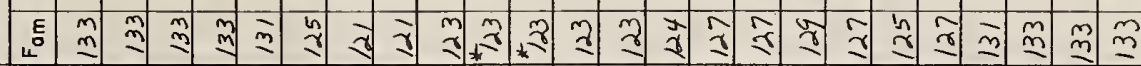

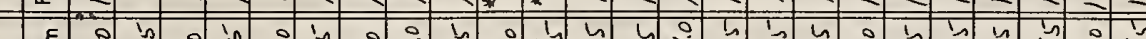
至 हE

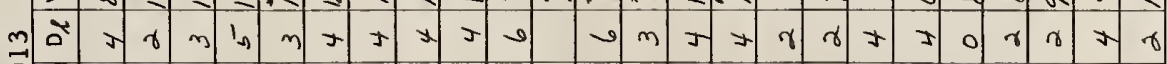

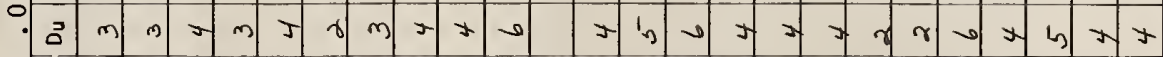

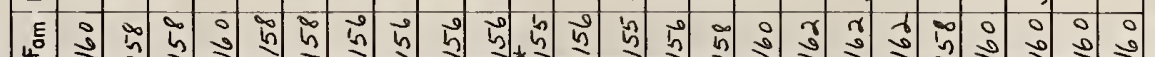




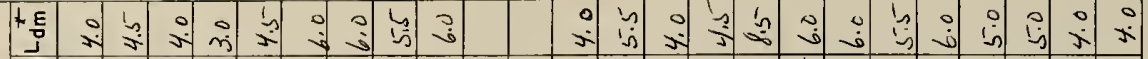

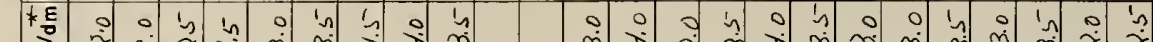

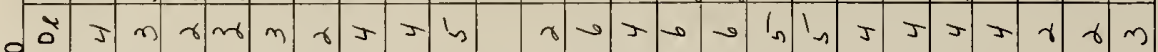
जे

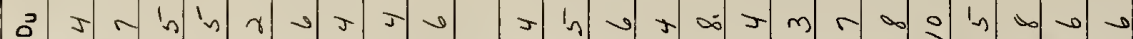

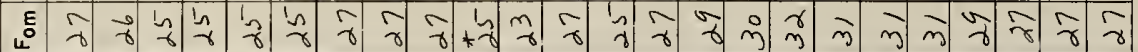

*E

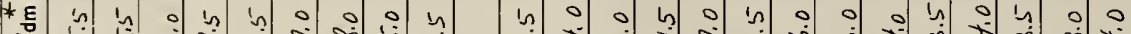

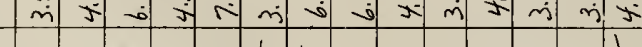

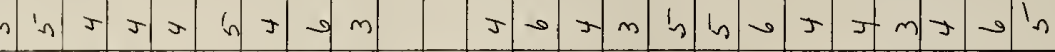

in

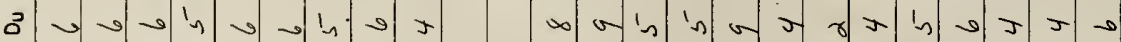

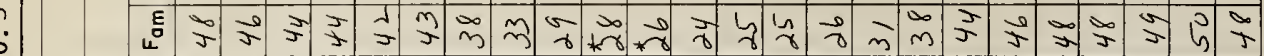

*E 북 *E

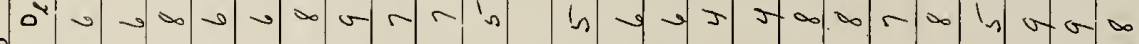

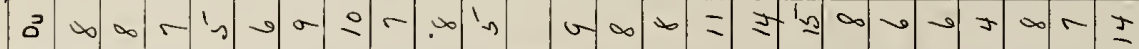

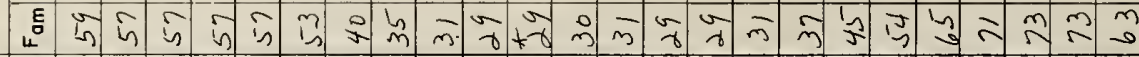

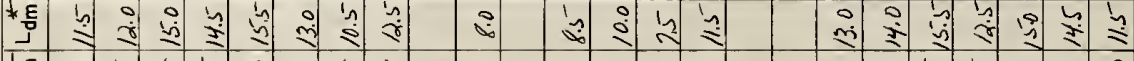

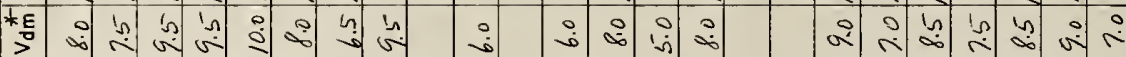

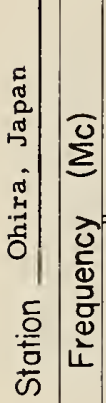

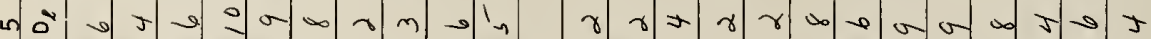

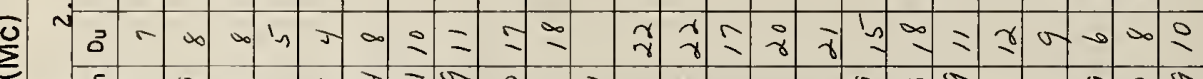

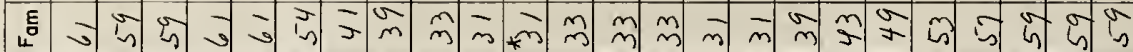
*E tE 0 l $>$ í.

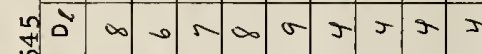
$\pi \rightarrow \ln \infty \rightarrow \infty \rightarrow \infty$

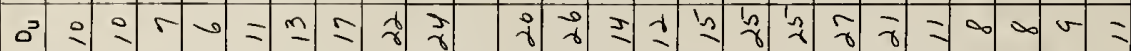

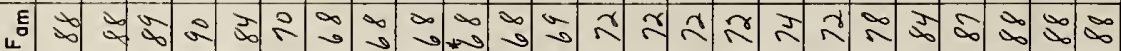

$\frac{1}{2}$

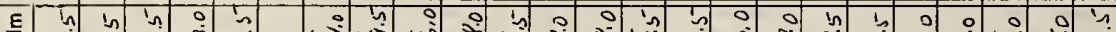

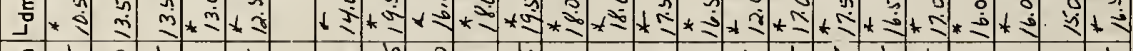

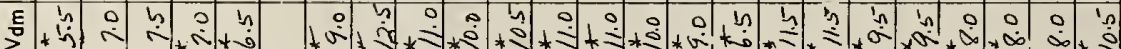

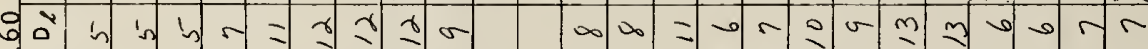

$\frac{0}{\alpha}$

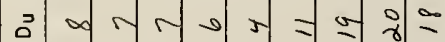

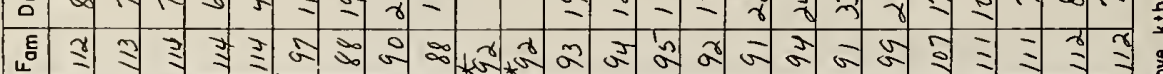

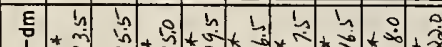

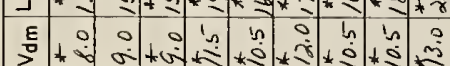

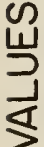

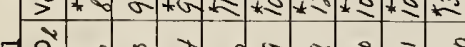

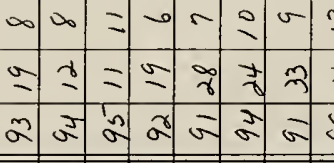

능 जी

\section{$\Rightarrow \rightarrow r \rightarrow m \rightarrow \infty \infty \infty$}

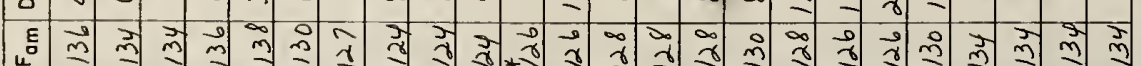

E

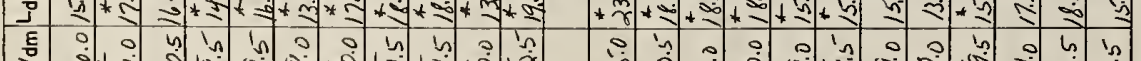

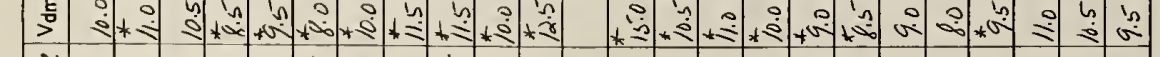

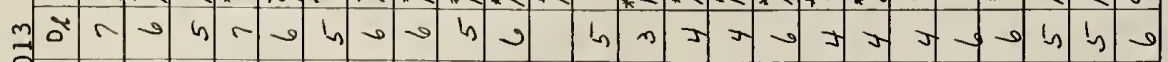

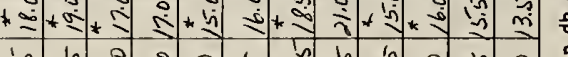

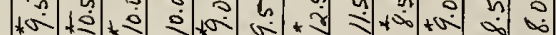




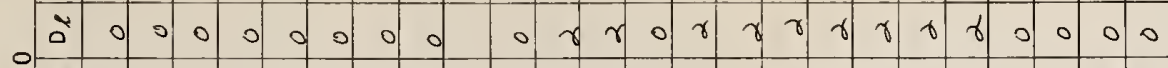
ก

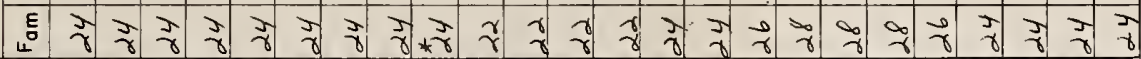

政

E

0 r.

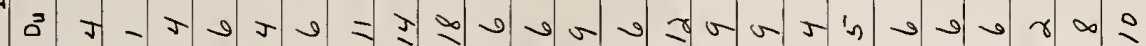

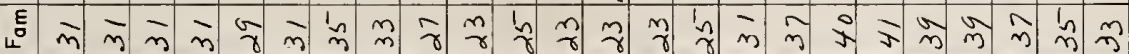

o

E

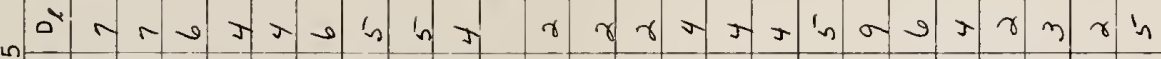
כ a

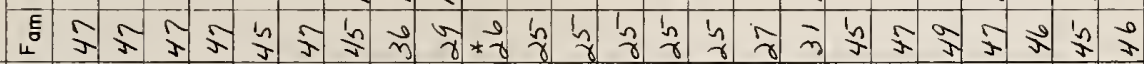

ก.

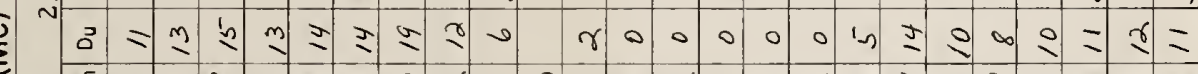

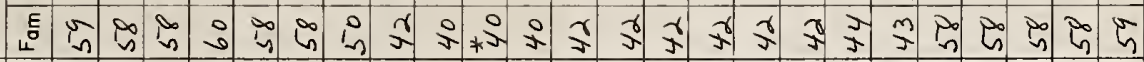
퉁

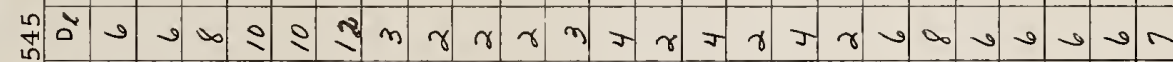

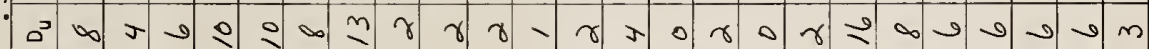

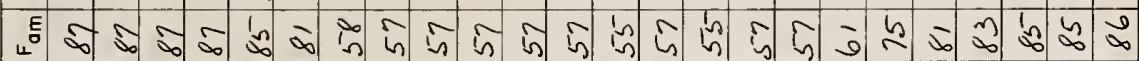

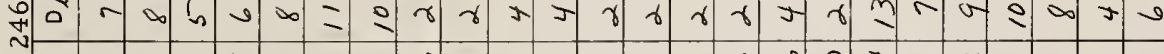

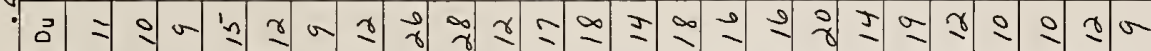
ए E

\section{西}




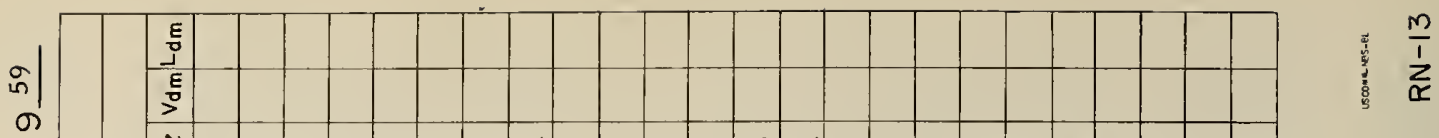

일

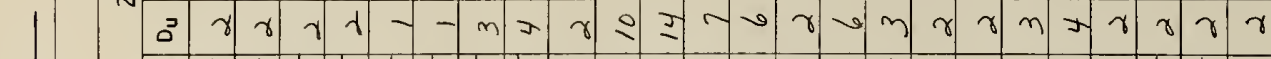

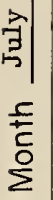

E

통

$\stackrel{E}{>}$

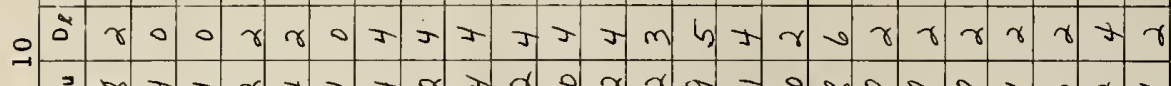
5 $\infty$ 小

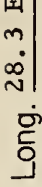

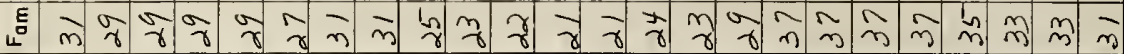

E

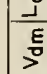

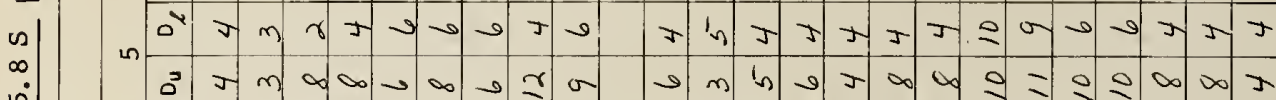

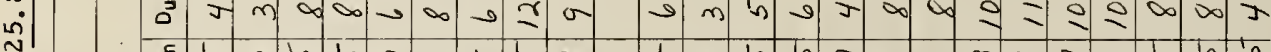

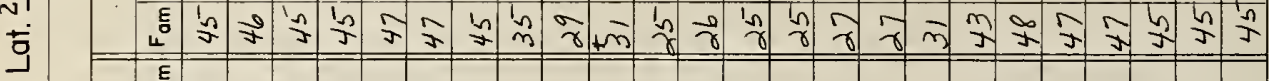

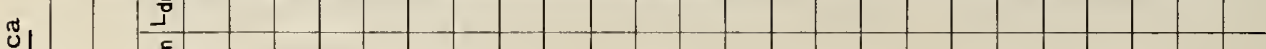

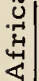

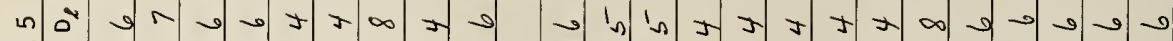

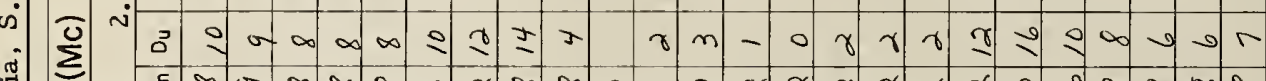

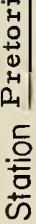
हE

उั

吾

这

in

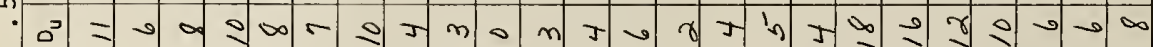

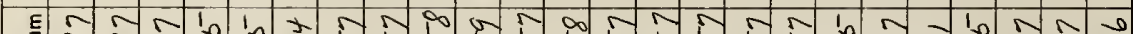

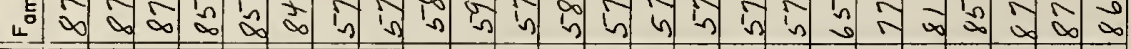

状

통

$>$

०

$\frac{\text { 음 }}{\text { ㄸ }}$

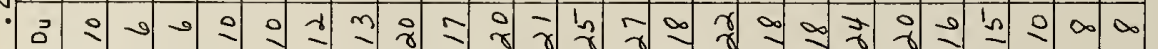

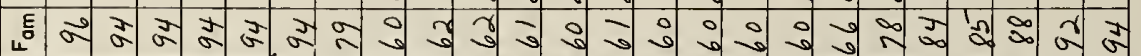

$+2$

님

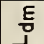

E

는

$m>$

(1)

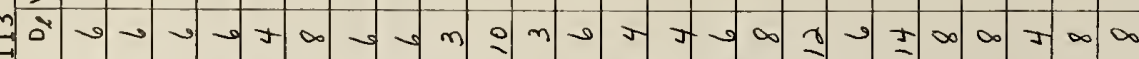

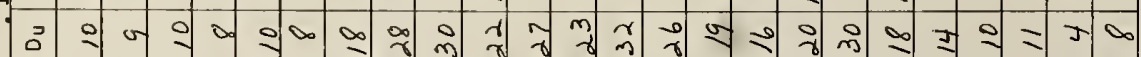

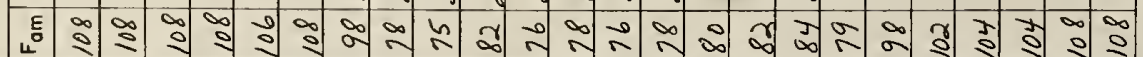

$\mathfrak{}$
$\frac{1}{1}$
$\frac{1}{5}$
$\frac{5}{2}$

-

E

틍

ป็

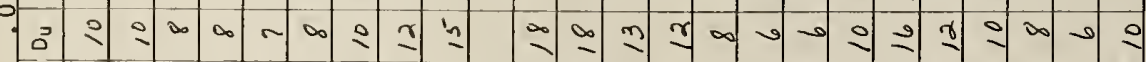

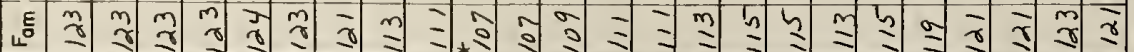

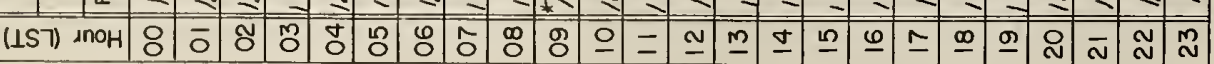

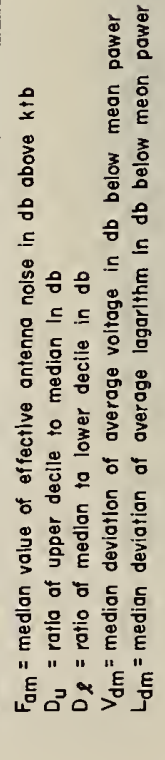




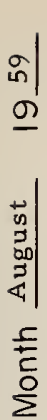<smiles>C1CC2CCC12</smiles>

s.

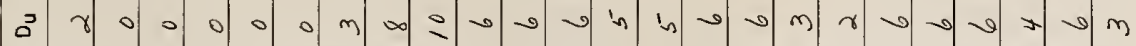

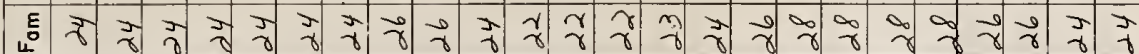

\begin{tabular}{c|c|c|}
\hline$E$ \\
\hline \\
\hline
\end{tabular}

वे $\gamma$ -

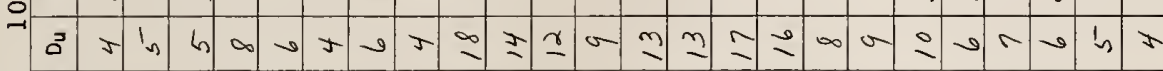

비

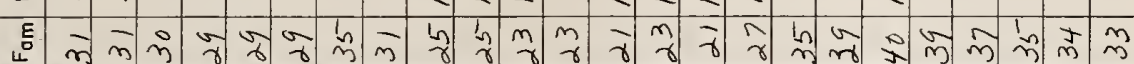

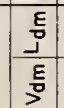

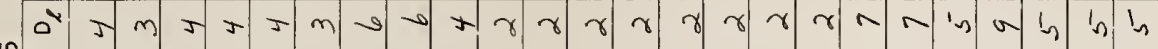

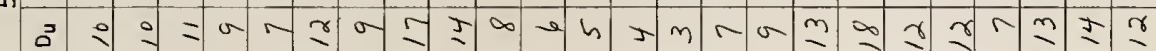

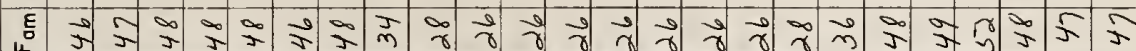

틈

แ

ए

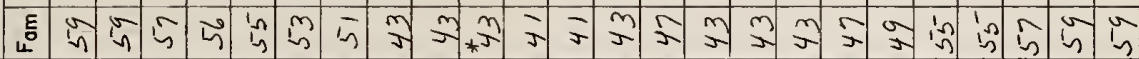

$\frac{\varepsilon}{9}$

हE

no

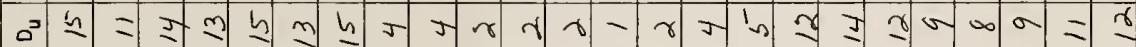

द

$\frac{1}{2}$

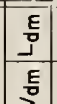

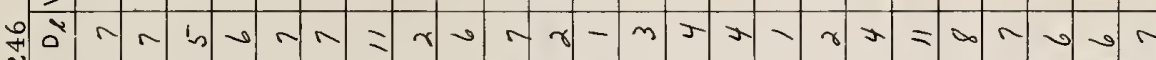

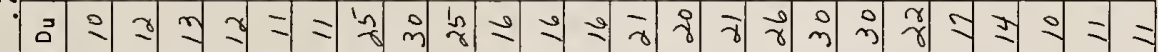

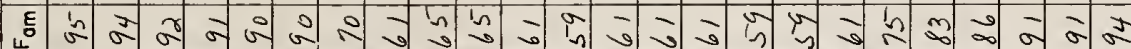

틈

$\frac{1}{5}$

峁

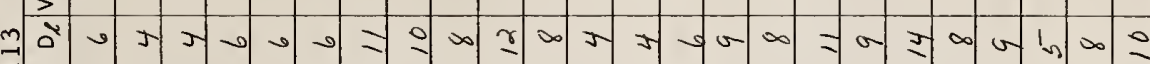

(1)

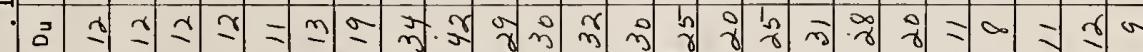

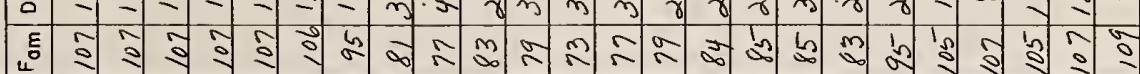

$\frac{1}{5}$
운
1
I
늘

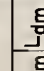

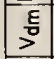

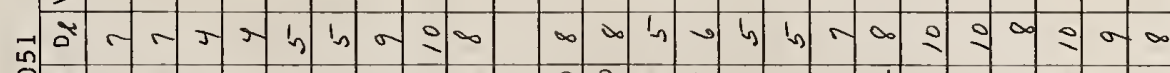

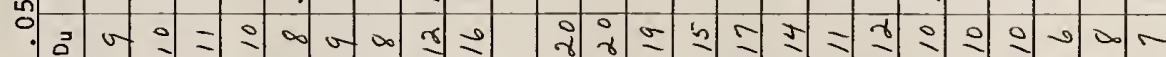

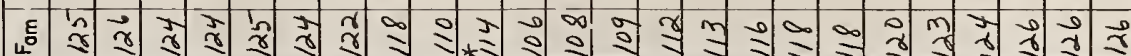
1 L

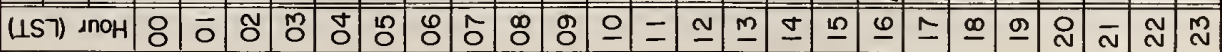




\section{in}

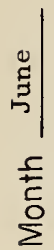

年

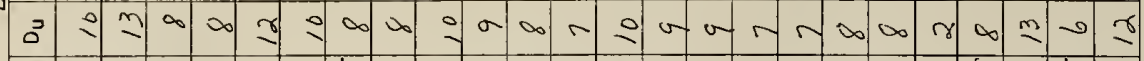

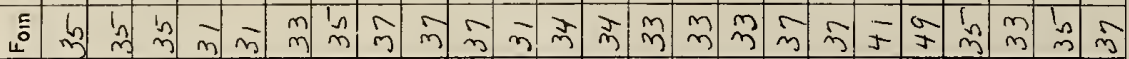

E.

E

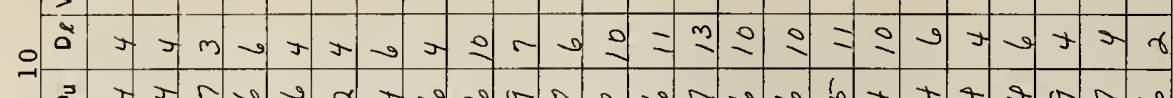

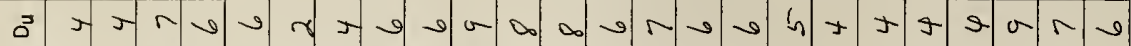
3
$\infty$
0

is

s.

:

छे

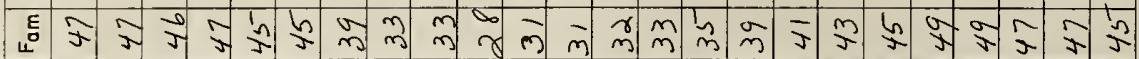

$-1$

至

$\stackrel{5}{\frac{E}{5}}$

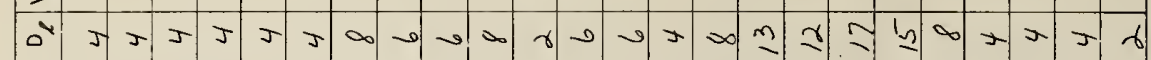

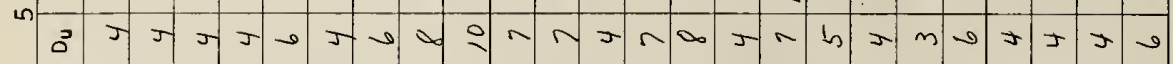

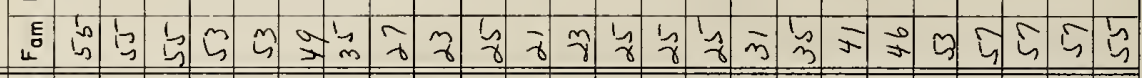

E्ञ

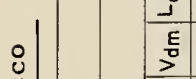

茫

i

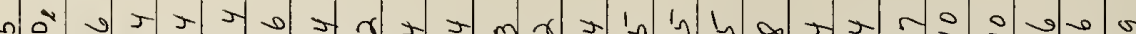

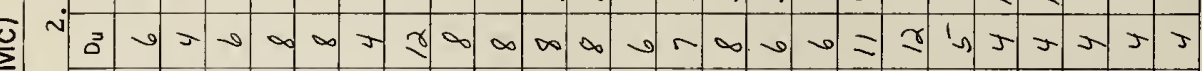

एह ชิ

얻리

ᄃ

음

मी

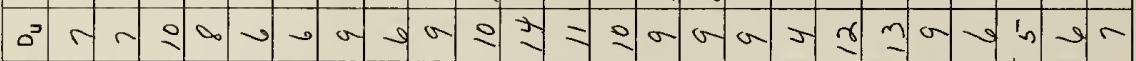

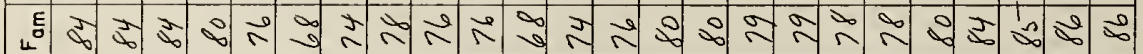

능
$\frac{1}{2}$
0
$\frac{0}{2}$

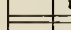

E

퉁

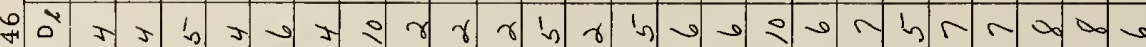

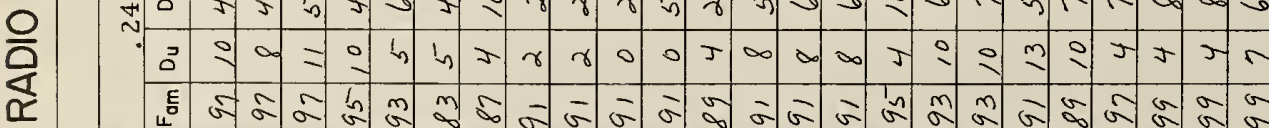

u

崫

$+\frac{1}{3}$

틍

$m \stackrel{\text { E }}{2}$

$\stackrel{m}{\exists}$

$\frac{1}{2}$
0
$\frac{1}{1}$
$\frac{1}{5}$
$\frac{1}{2}$

ב

E

톡

퉁

मी

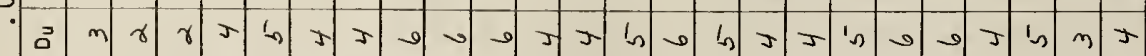

1 -

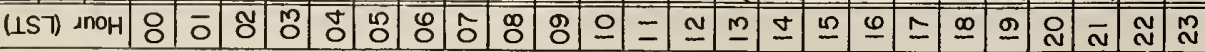

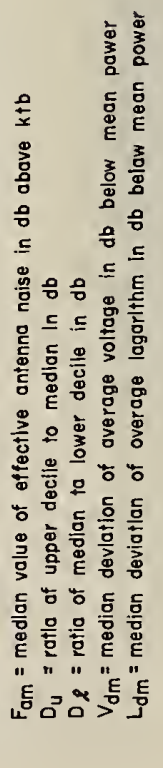


E

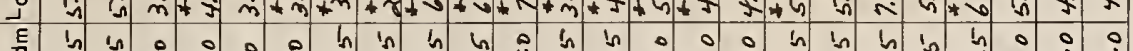

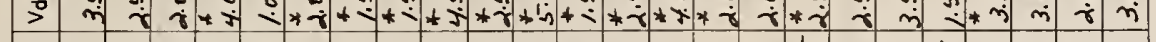

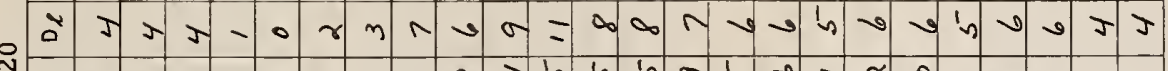

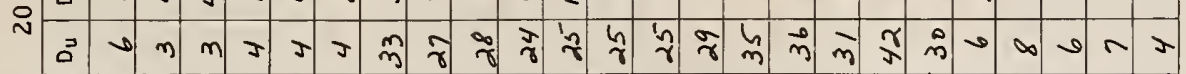

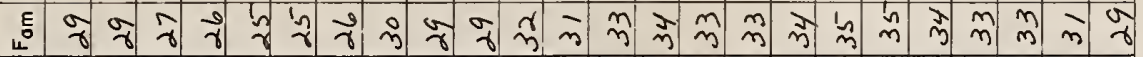

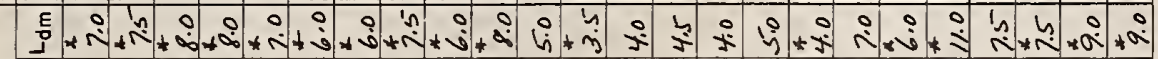
E 00 o 00 in 0 n $y$ i. 0 in 0 in 0 in 00 in 0 i 0 o 0

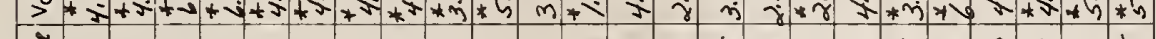

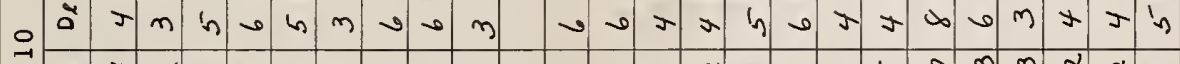

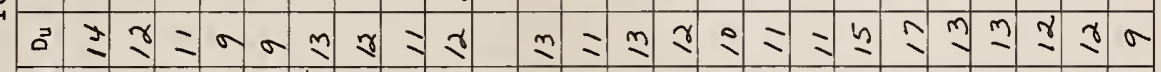

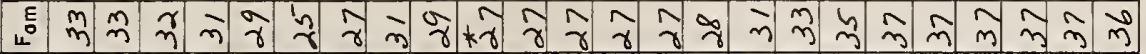

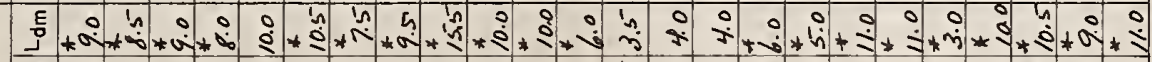

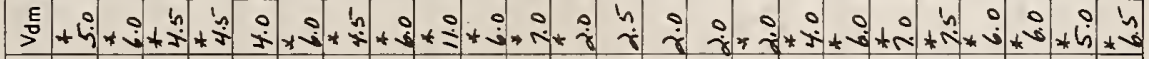

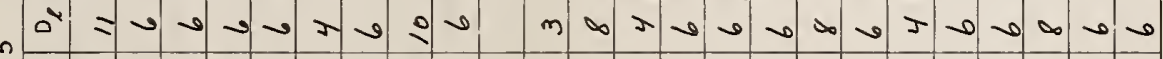

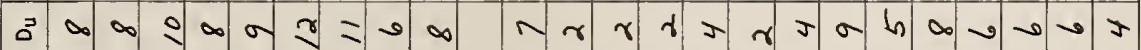
ह

క

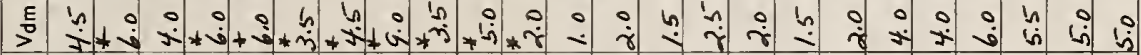

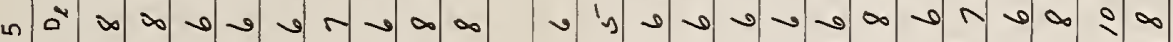
ง

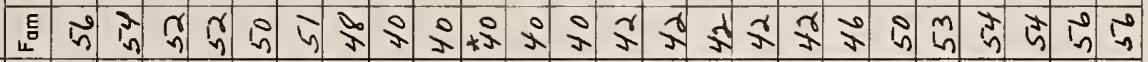

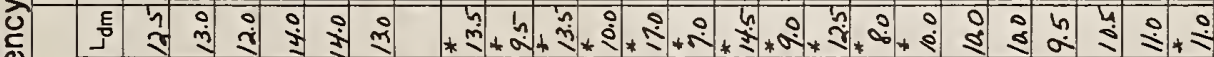

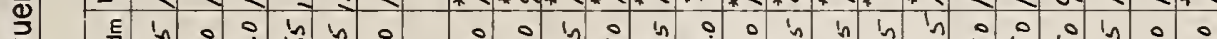

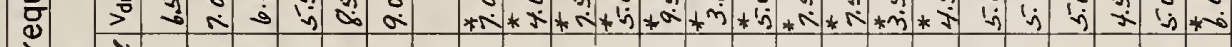
Lั

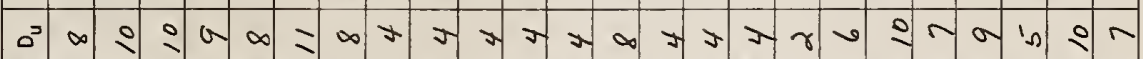
น

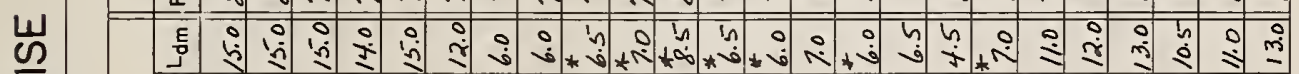
O $\quad$ E

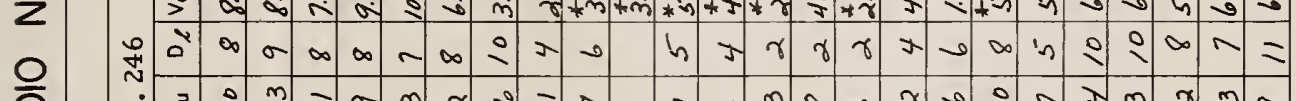

$\frac{2}{\frac{1}{4}}$

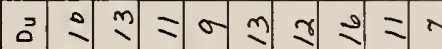

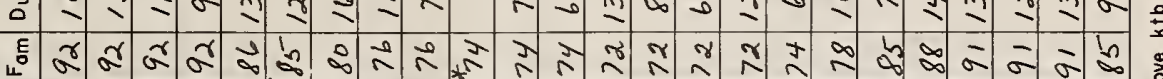

ह

แ

j.

E

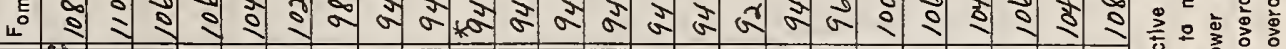

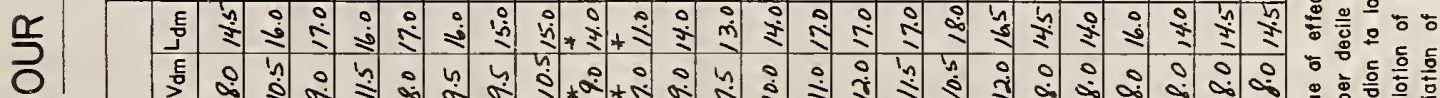

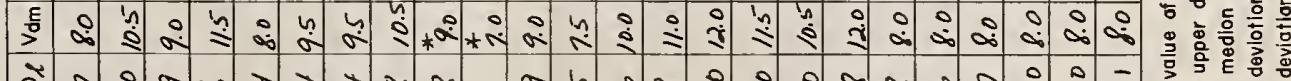
चु I E $\quad$ E

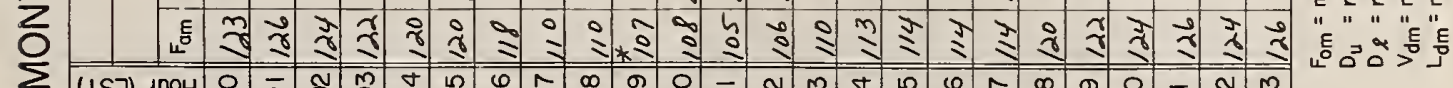

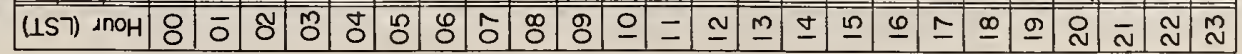


E

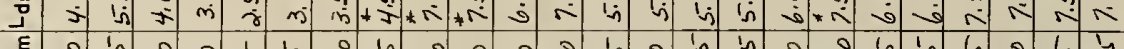

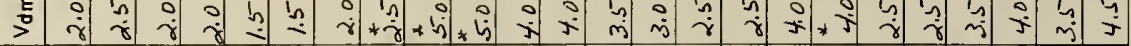
-

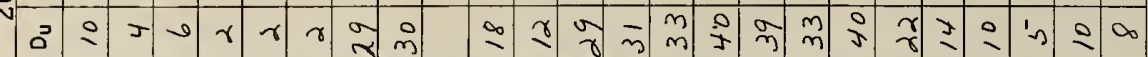

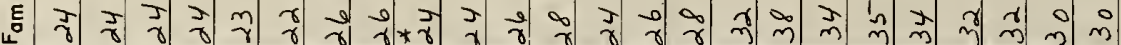
ह 0 G

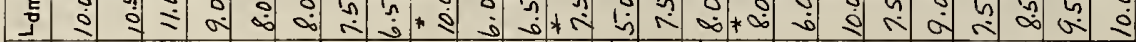

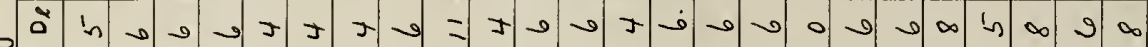

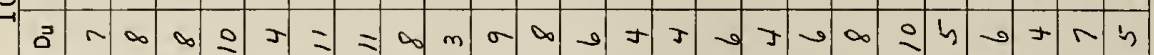

ผ J

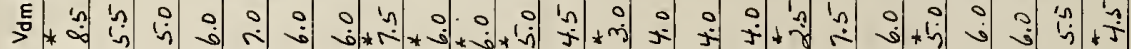

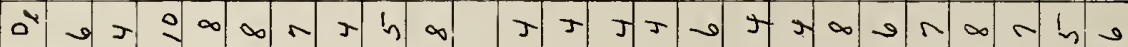

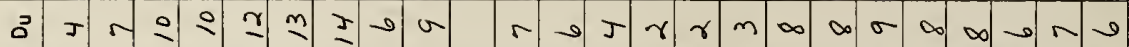
है

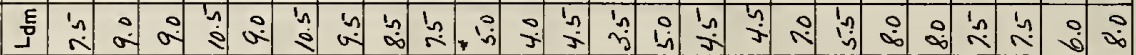

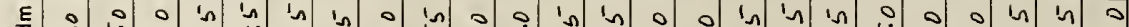

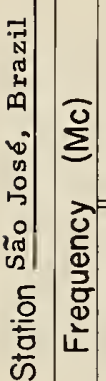

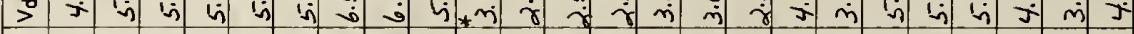

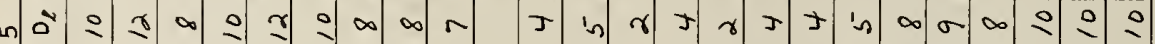

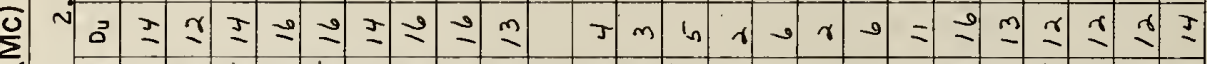
है

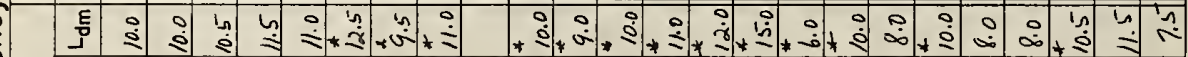

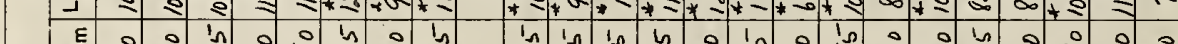

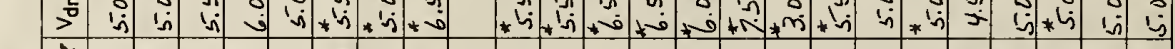

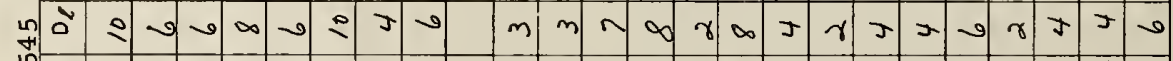

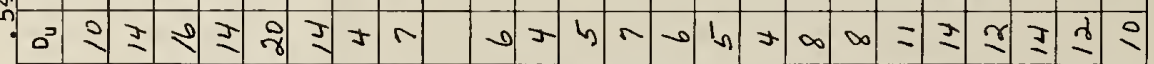
แ

$\frac{w}{2}$

$E$ E 00 o c n ว

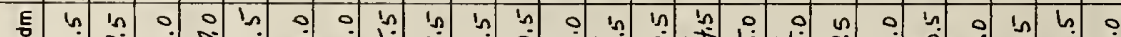

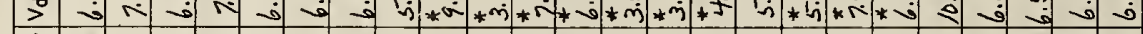

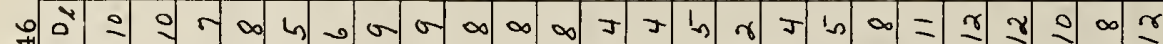

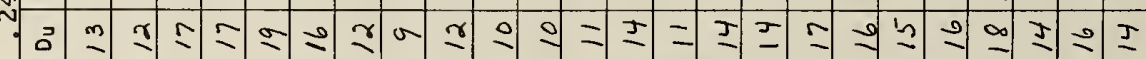
है E ป

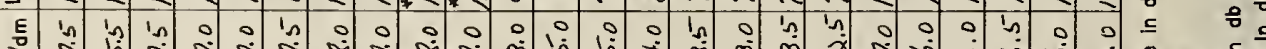

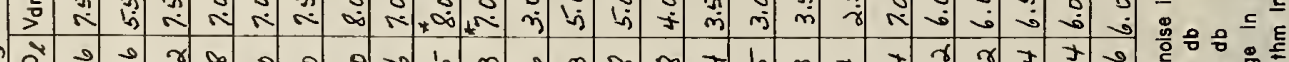
$\Rightarrow$ व

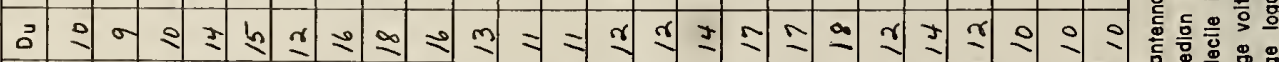

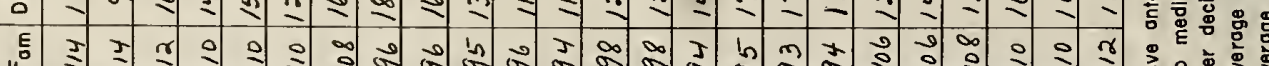
แ

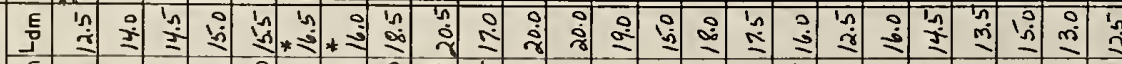

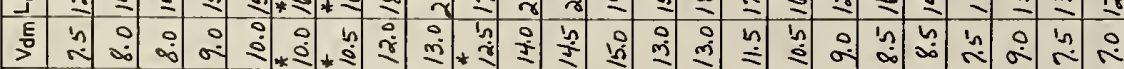
औี 要 
E >

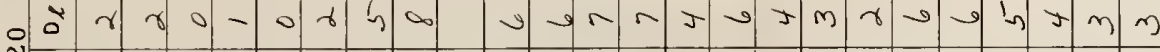

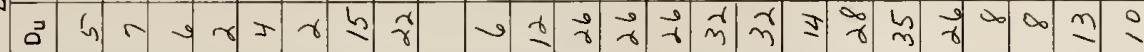

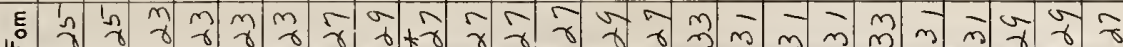

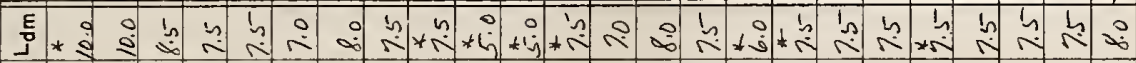

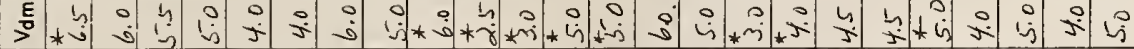

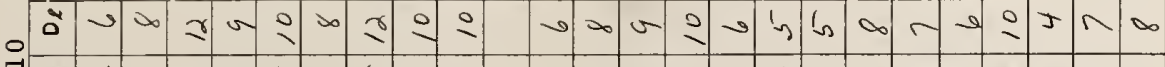

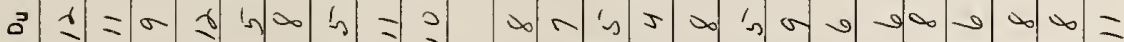

in

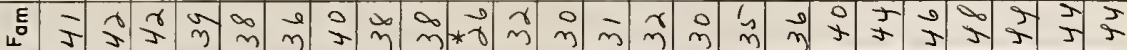

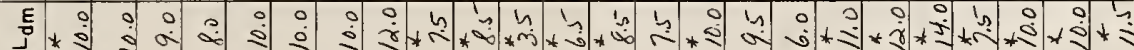

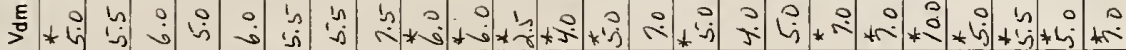

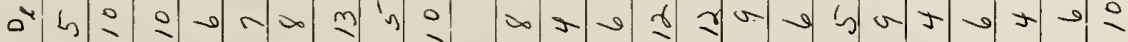

u

m.

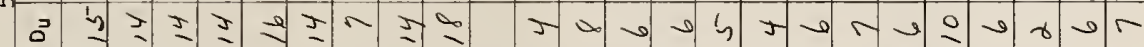

$\stackrel{m}{\sim}$

苛

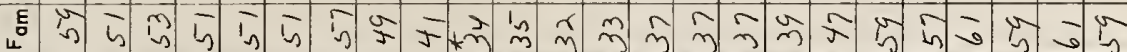

E

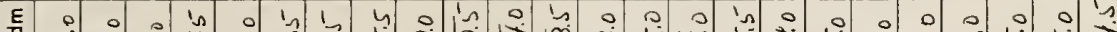

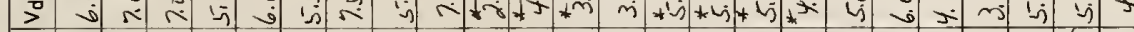

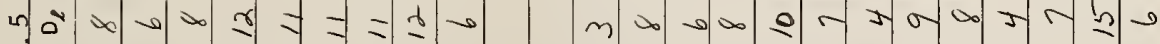

Yว 0 -

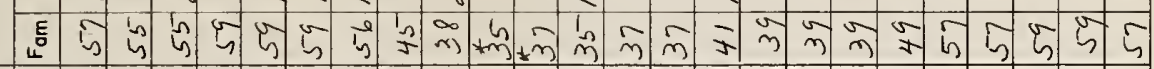

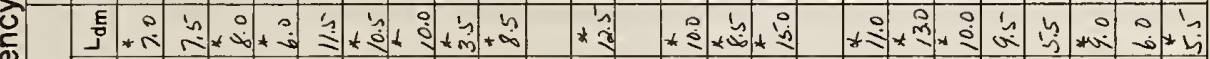

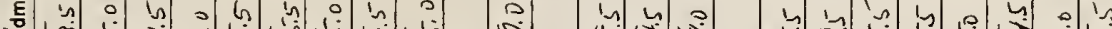

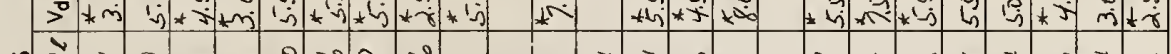
ํำ

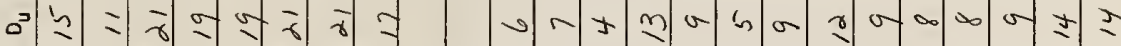
एँ

는

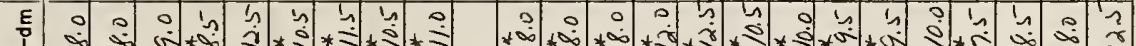

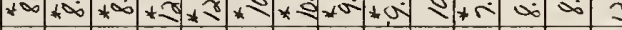

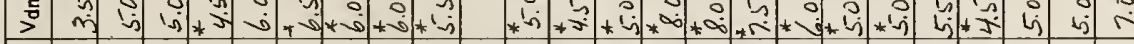

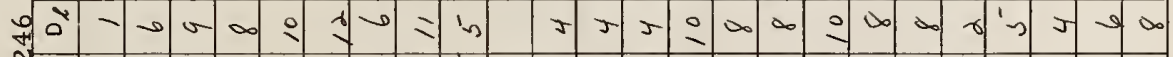
을 भे है

䜿我

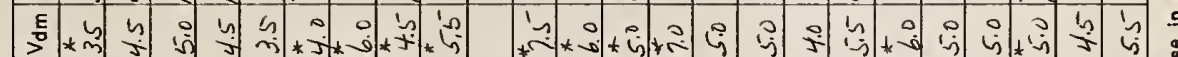

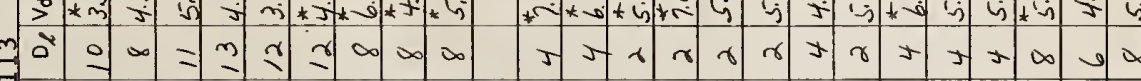
ว ป ह

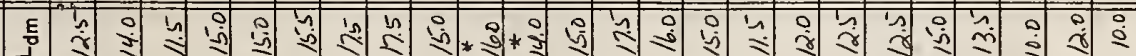
至

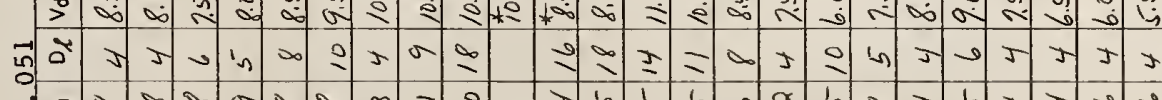

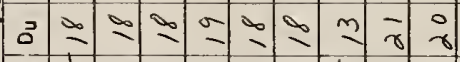


음

\begin{tabular}{|c|}
\hline E \\
\hline \\
\hline E \\
\hline D
\end{tabular}

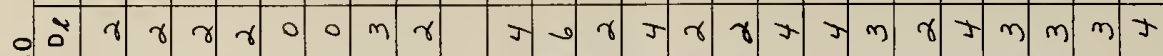

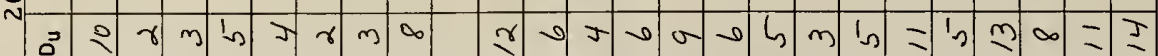

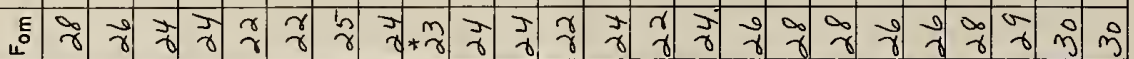

岢

달

$\frac{5}{2}$

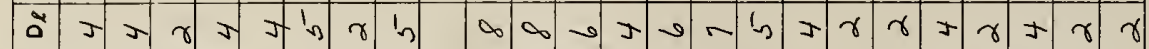

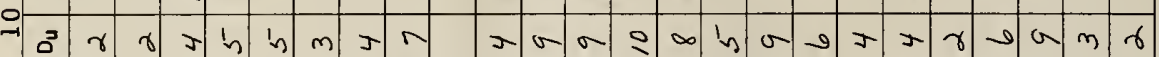

|x|

.

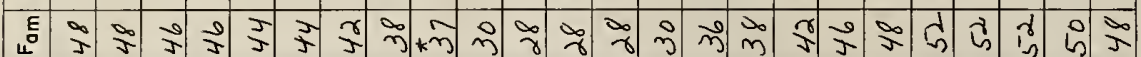

它

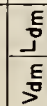

\begin{tabular}{c}
$z$ \\
$m$ \\
-1 \\
$\vdots$ \\
\hline
\end{tabular}

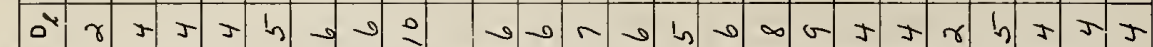

+5

离

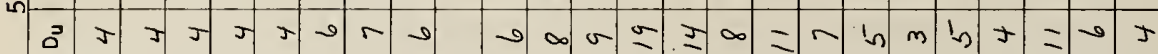

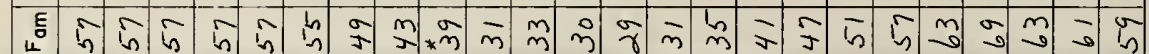

$\frac{E}{3}$

$\stackrel{5}{>}$

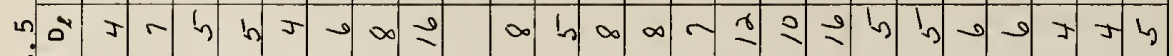
ड़

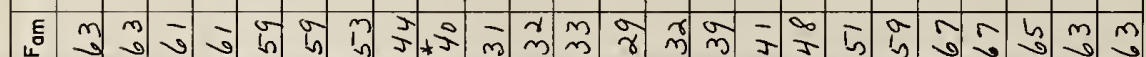
入े

ट

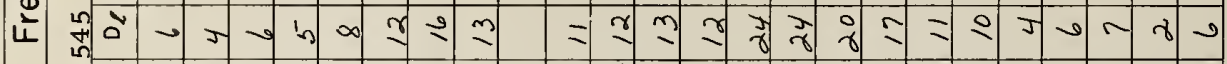

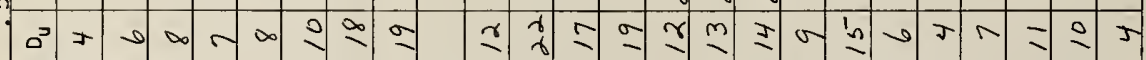

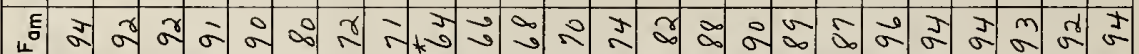

$\frac{\mathrm{m}}{\mathrm{O}}$

E

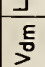

0.

을

है

s

E

崩

50

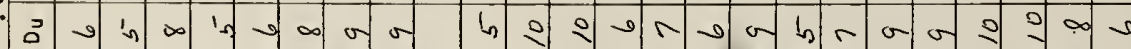

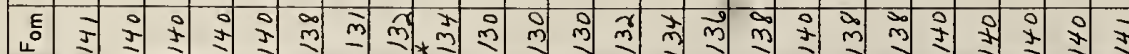

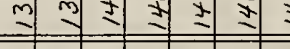

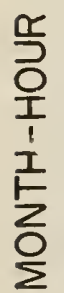

I

希

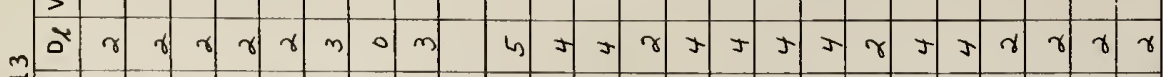

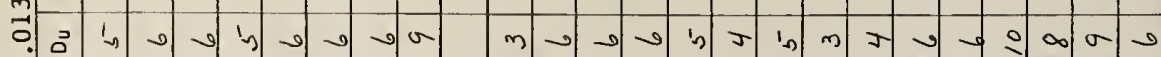

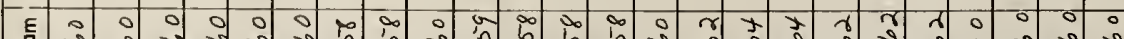

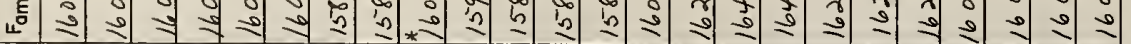

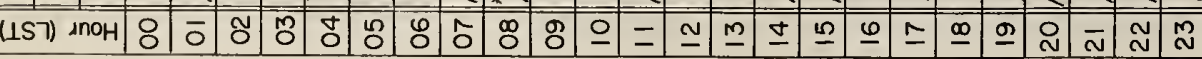

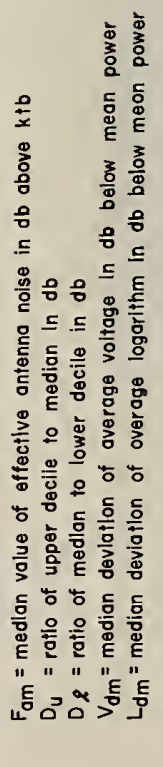




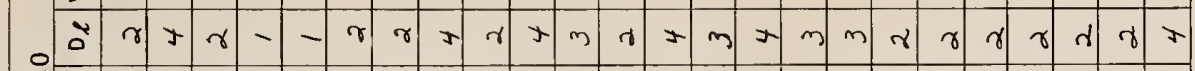

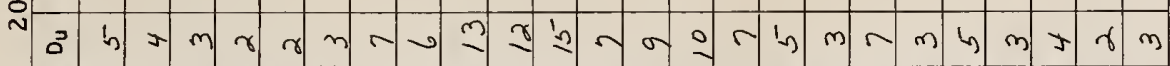

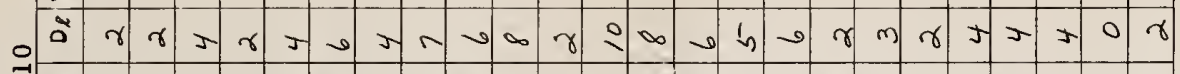

E्

통

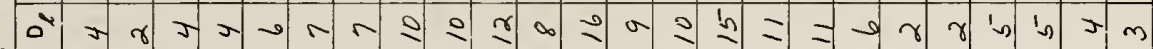

n) N

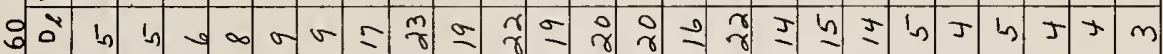




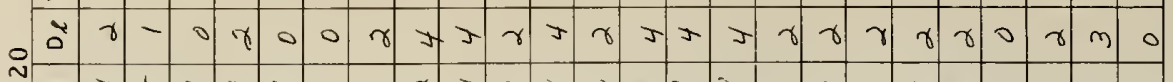

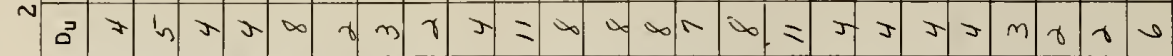

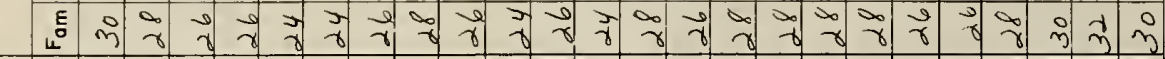

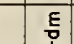

E

0 व

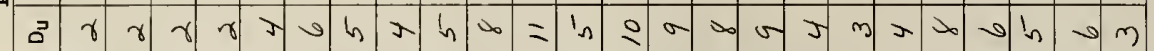

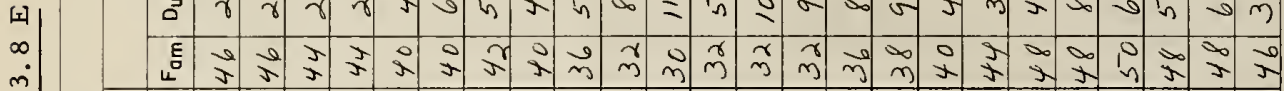

$\stackrel{\infty}{\infty}$

nा न म E

E

$m$

$-i$

5

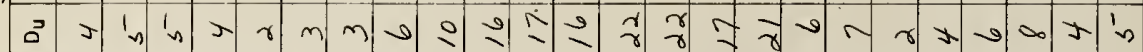
E E

帘

ก

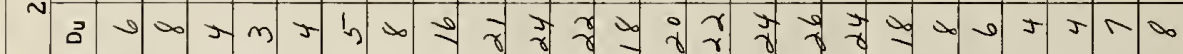
ह is

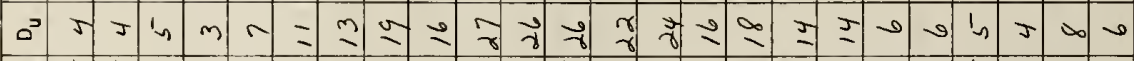
ए.

$\frac{w}{2}$ (1)

틍

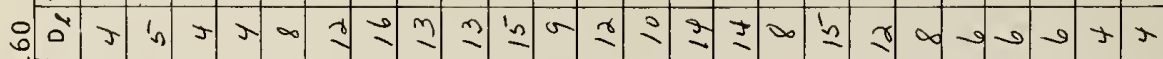

윰

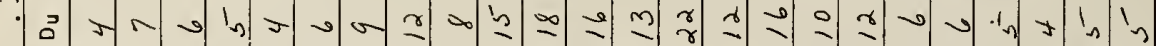
है E

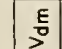

岁

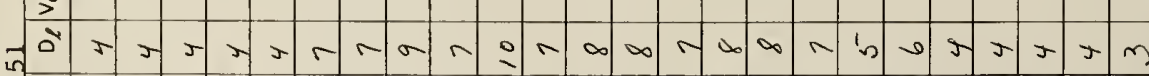
ว

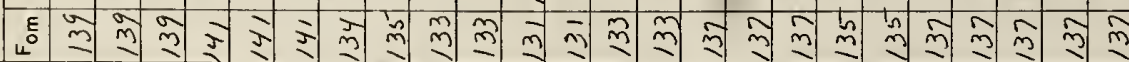
E 


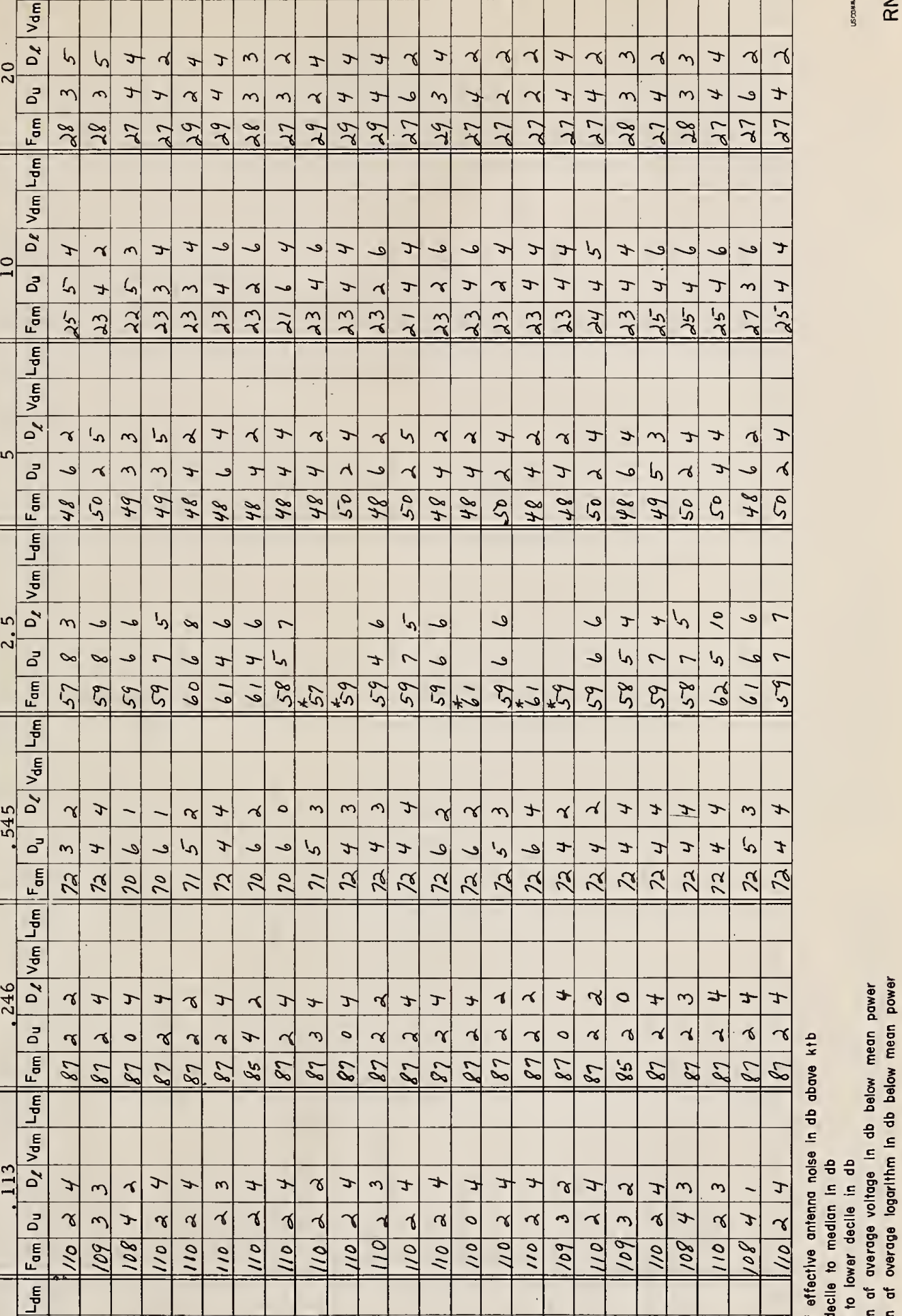

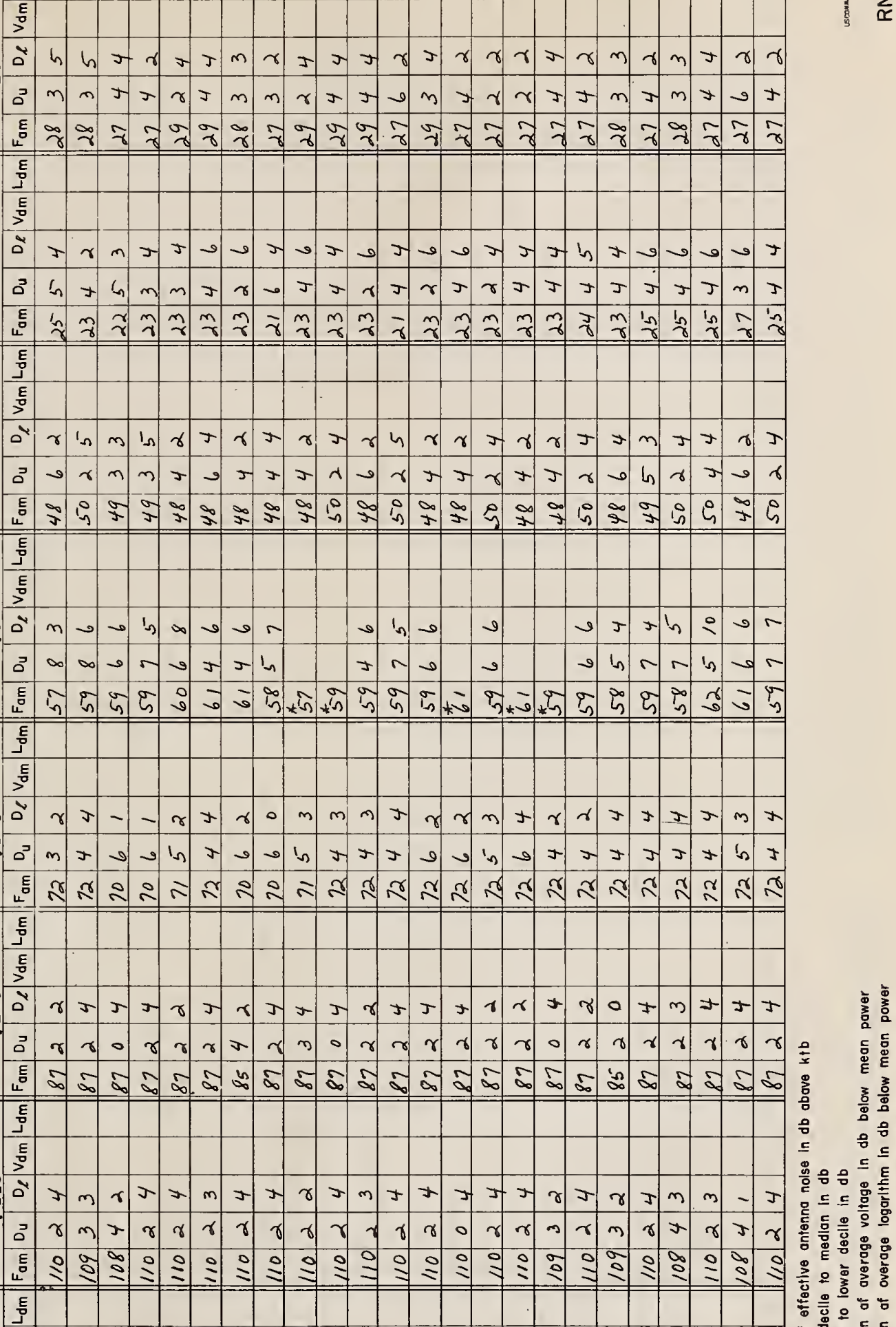

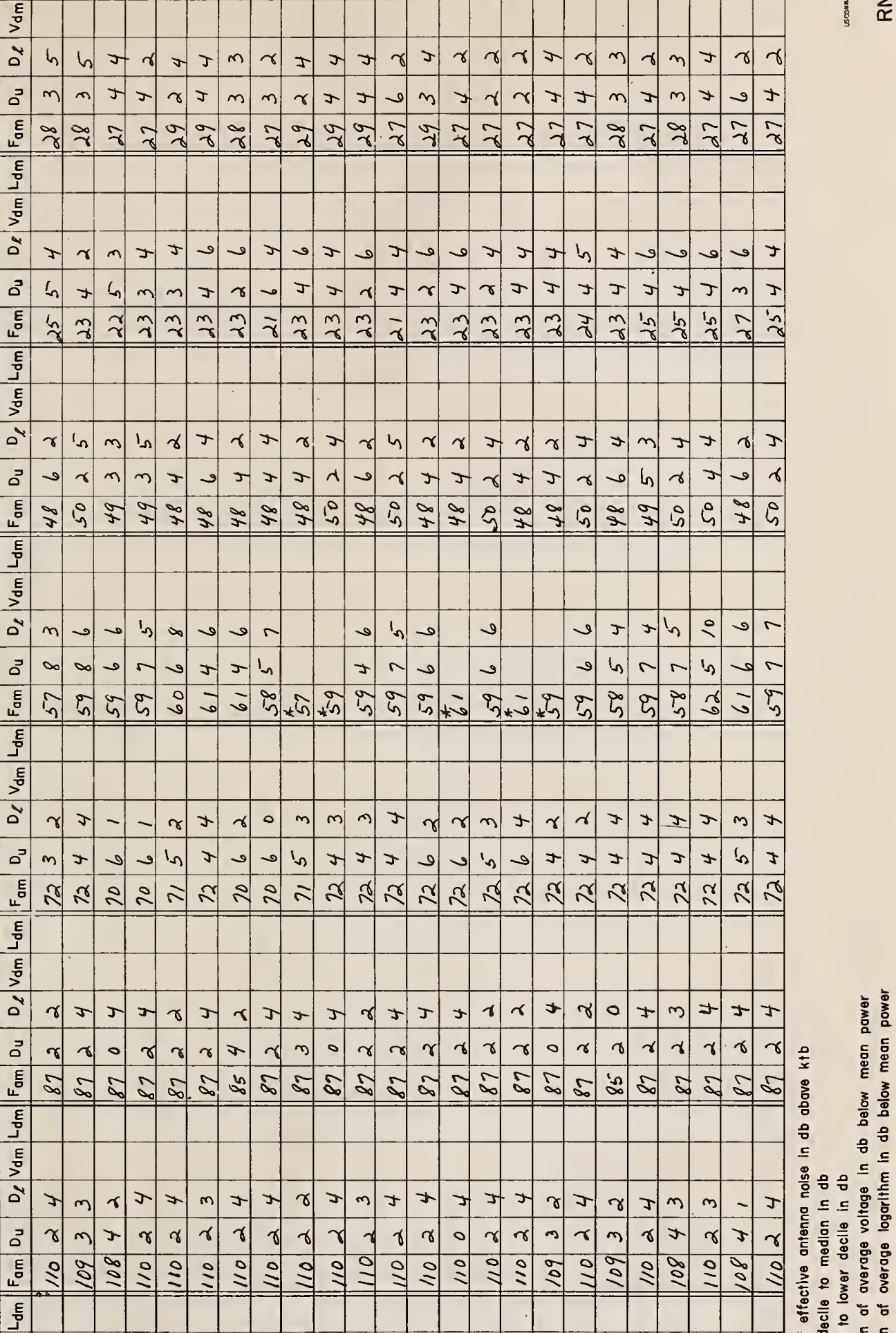

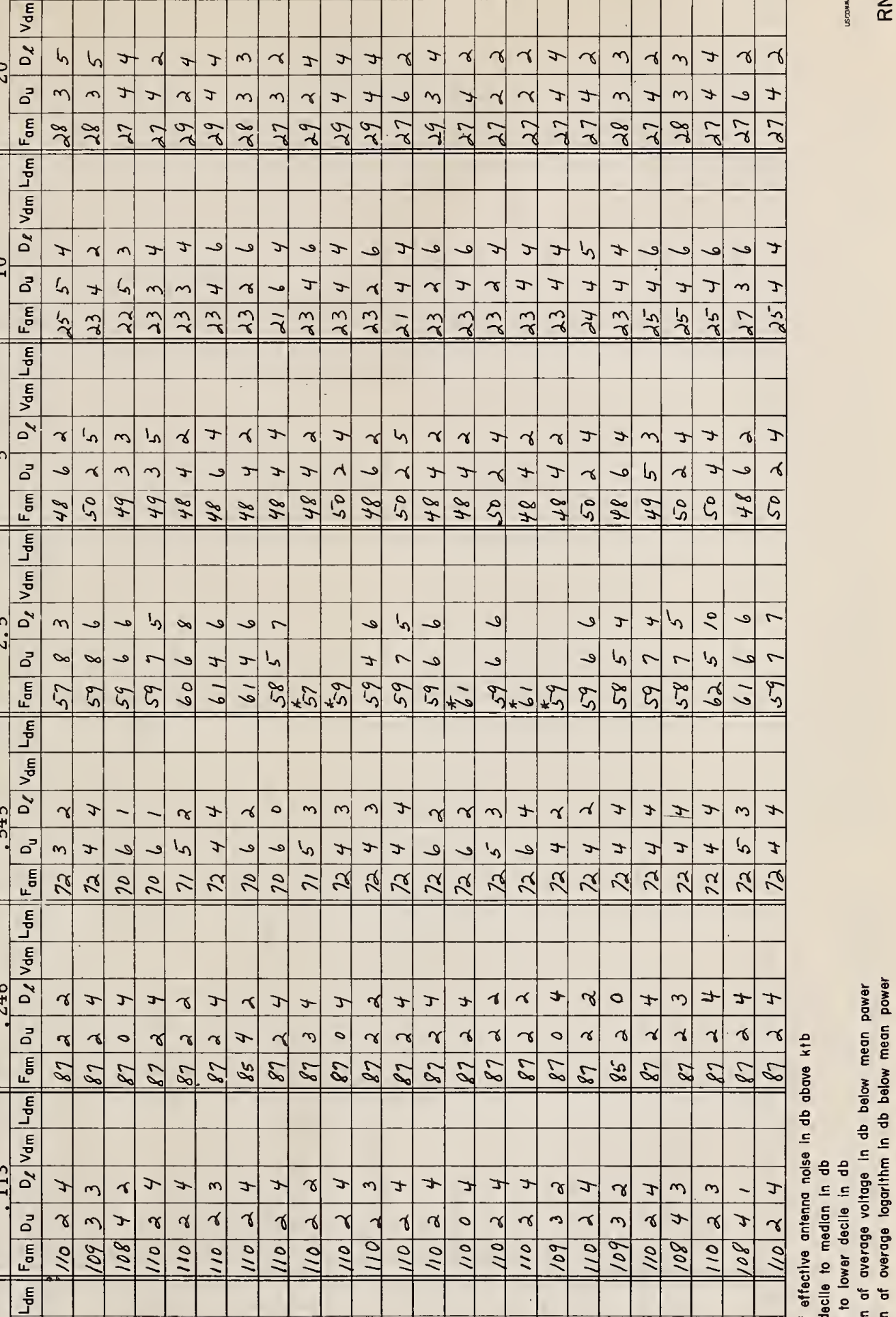

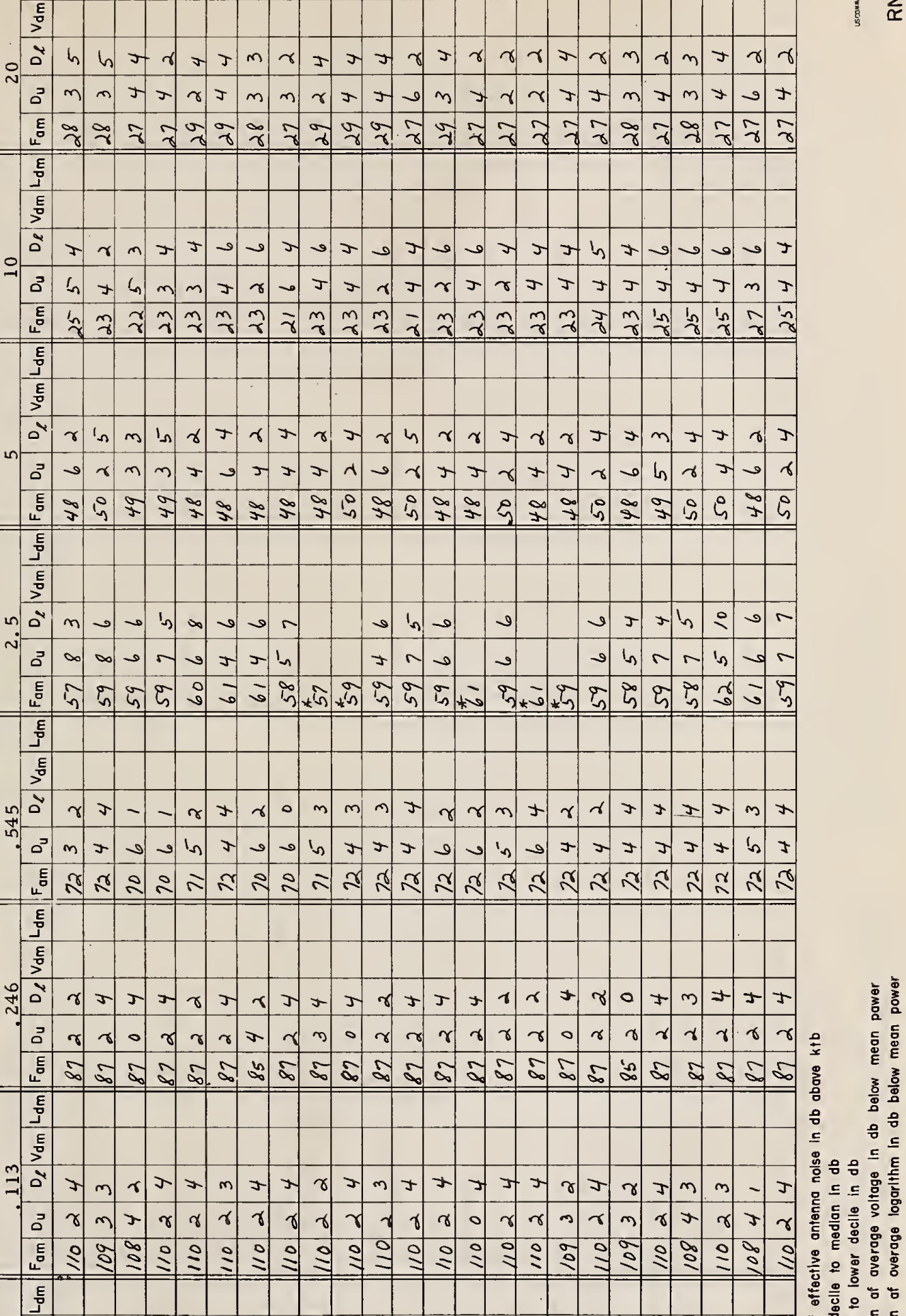

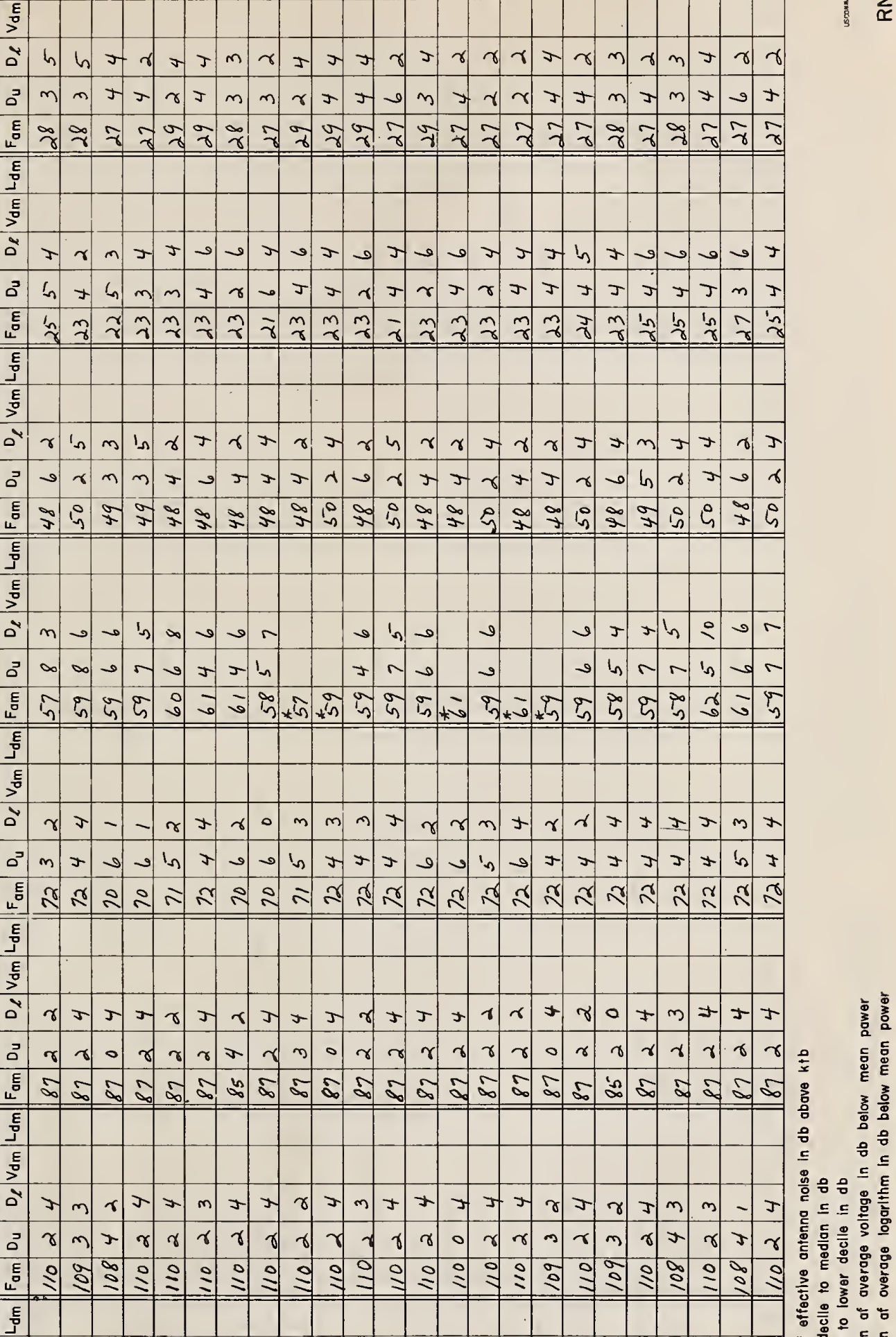

\section{E्}

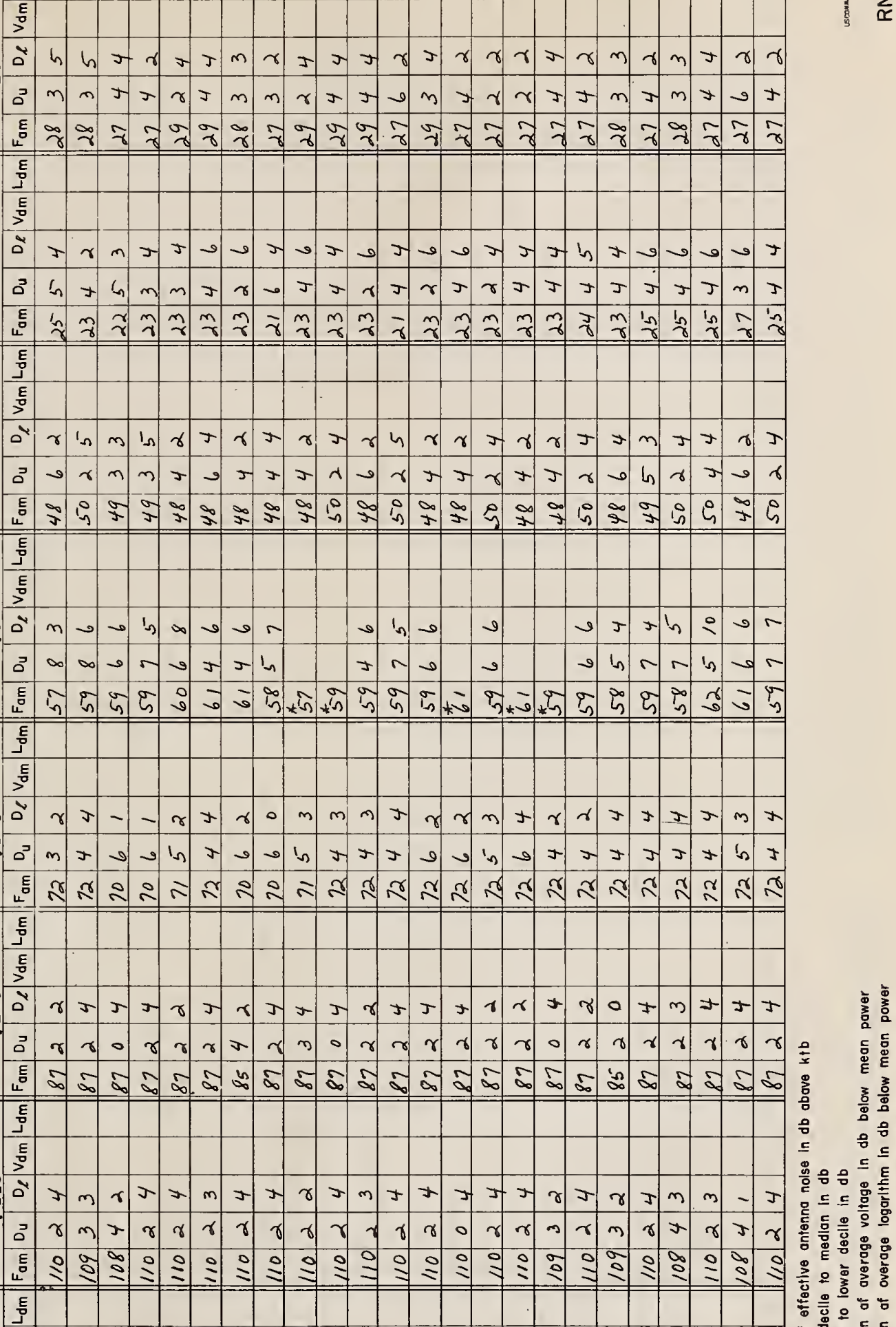

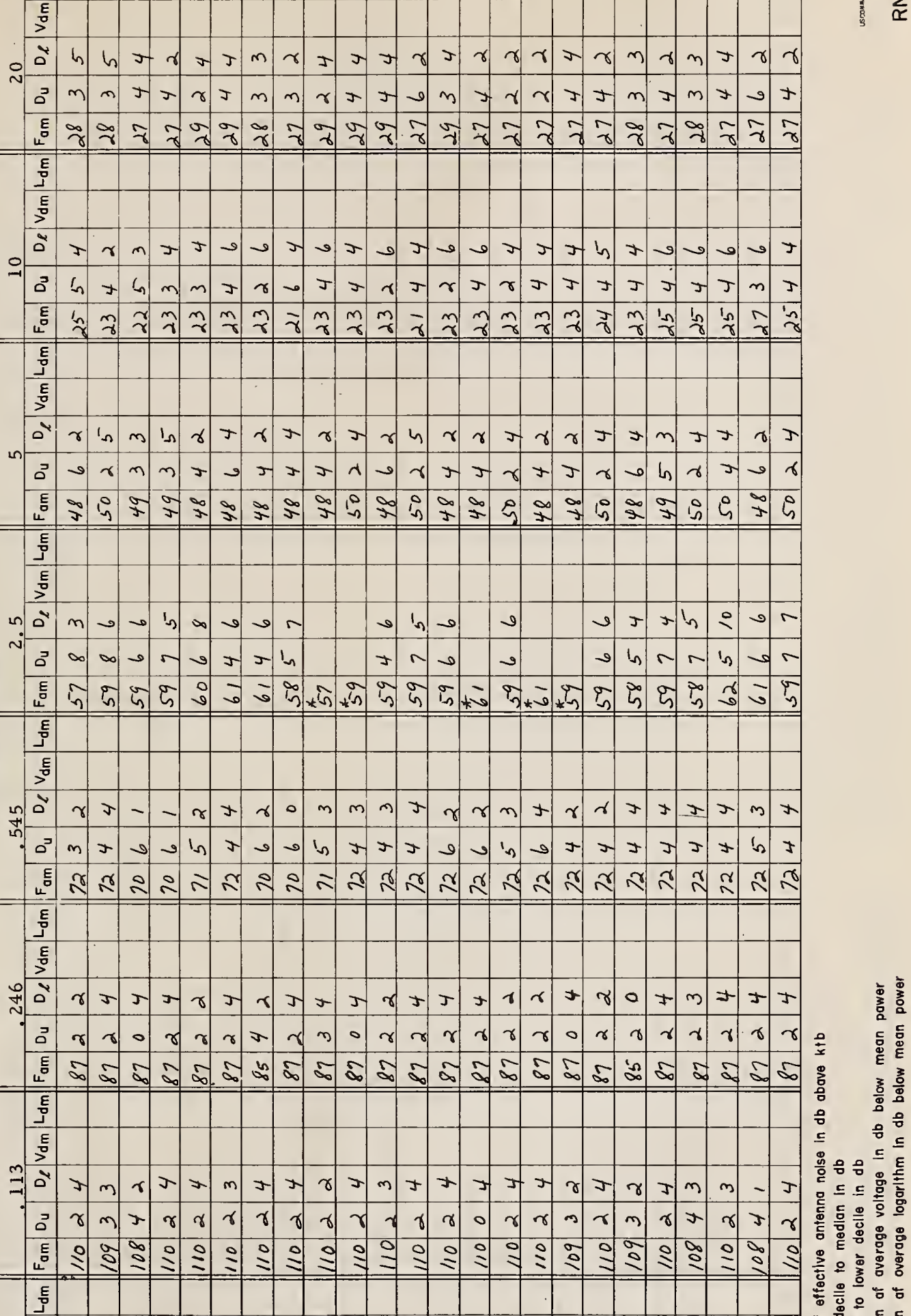

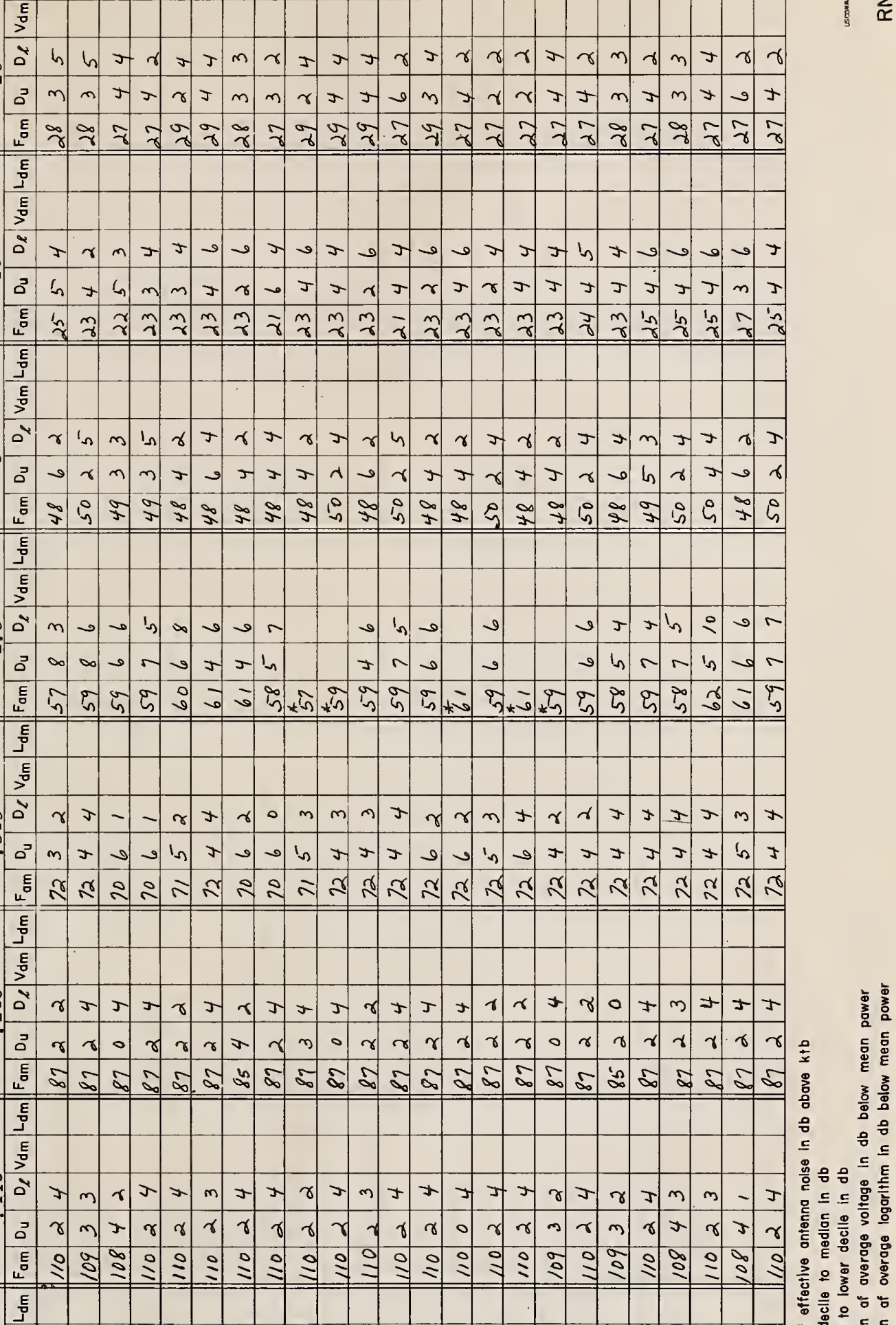
E

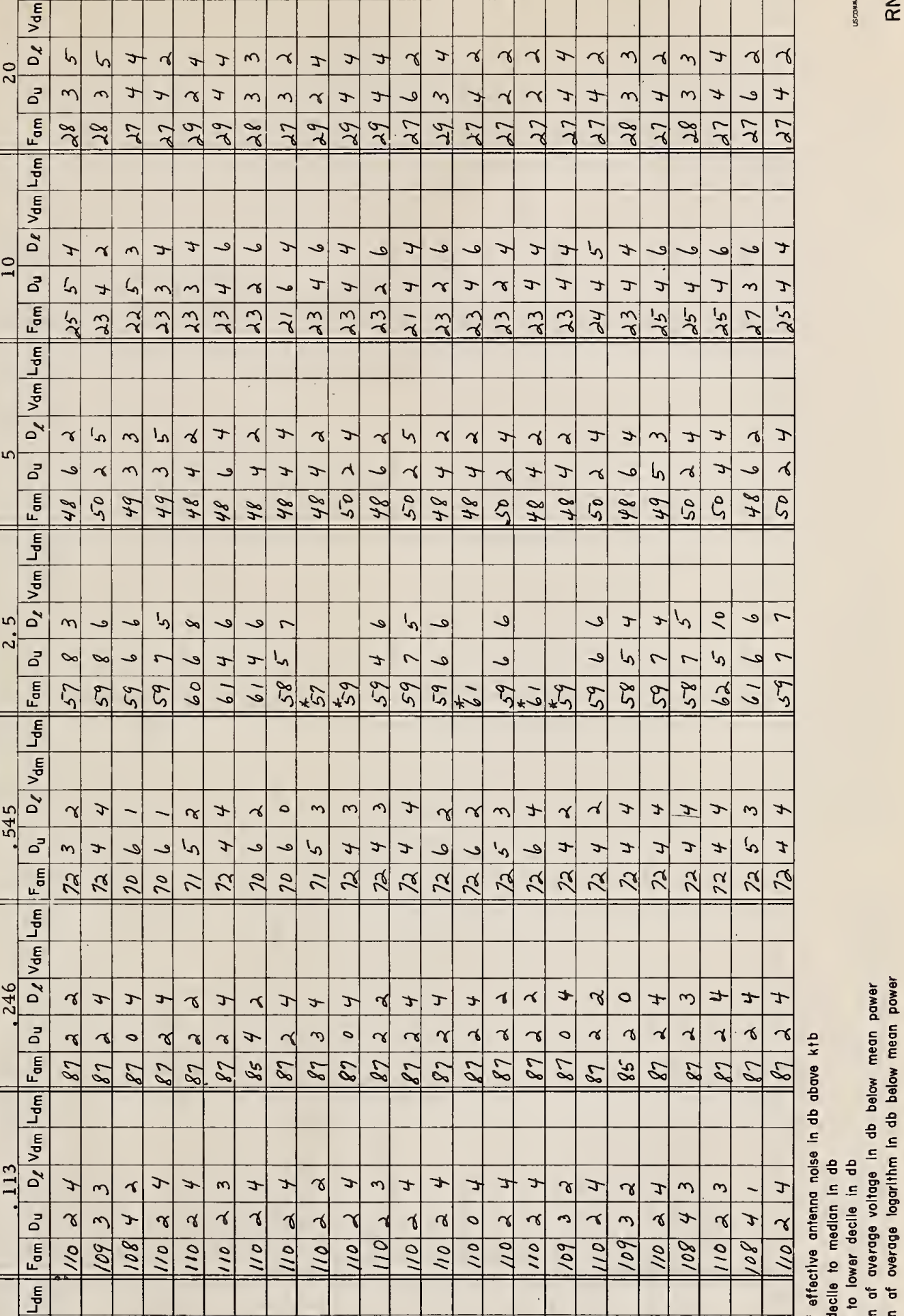

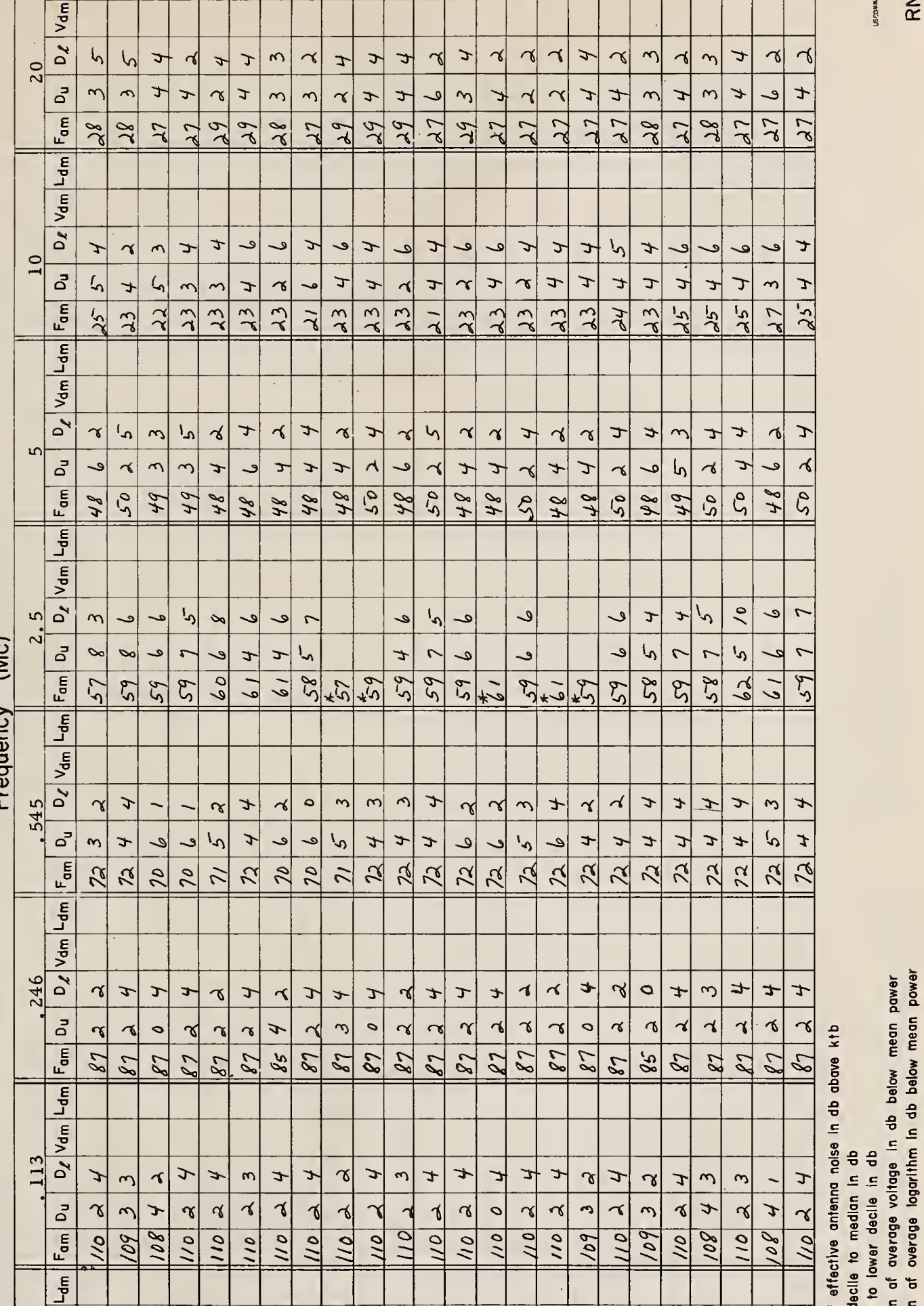

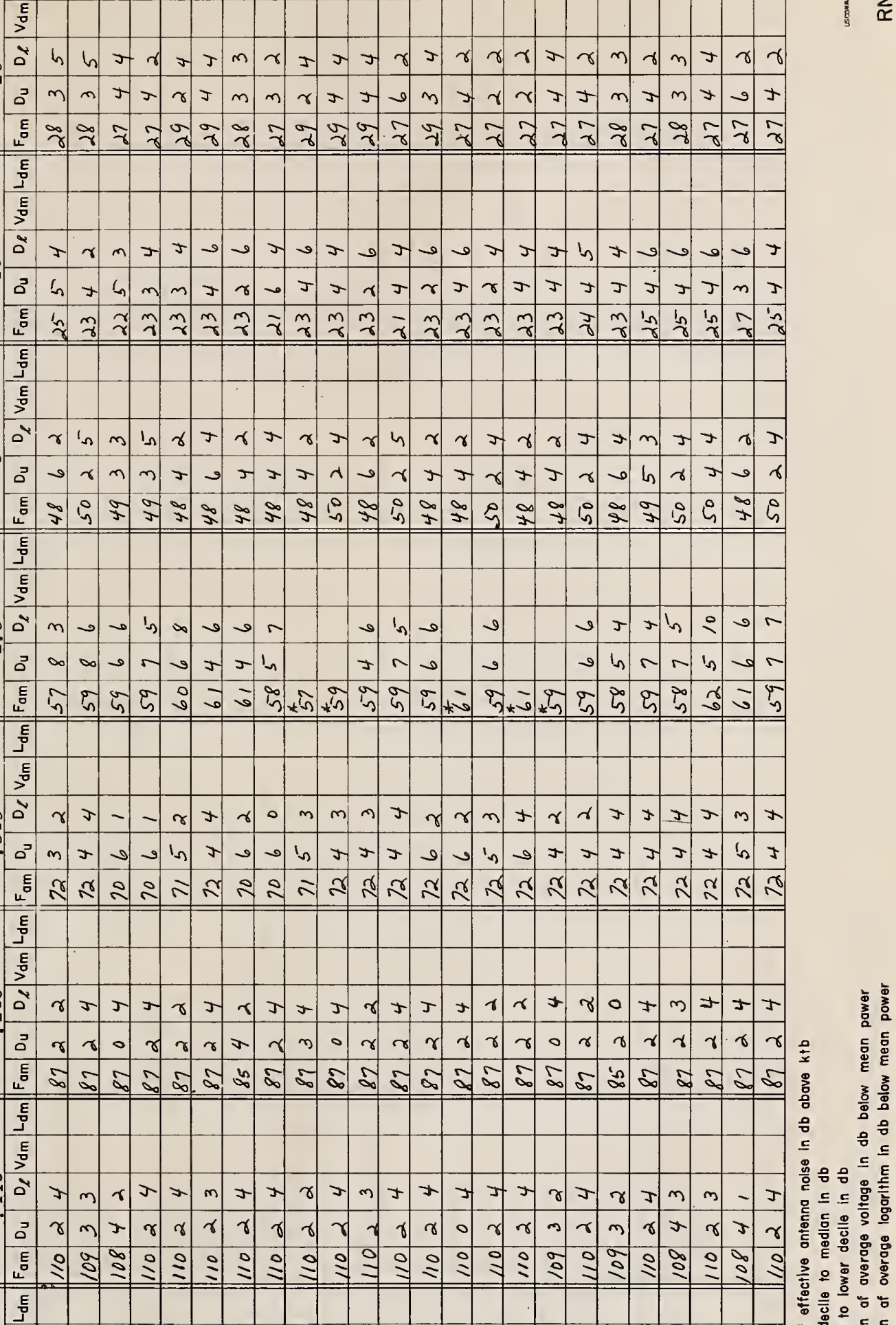
그

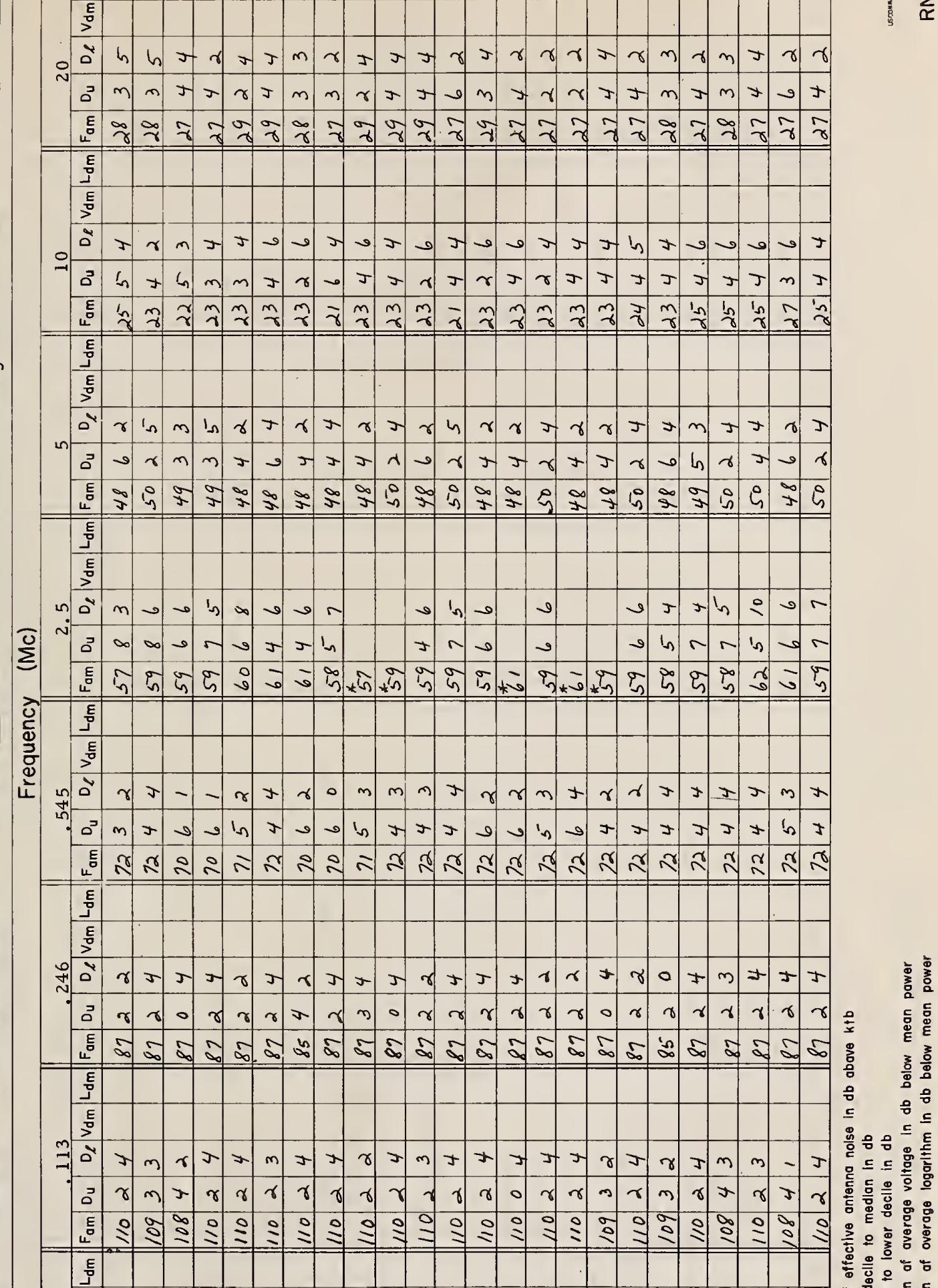

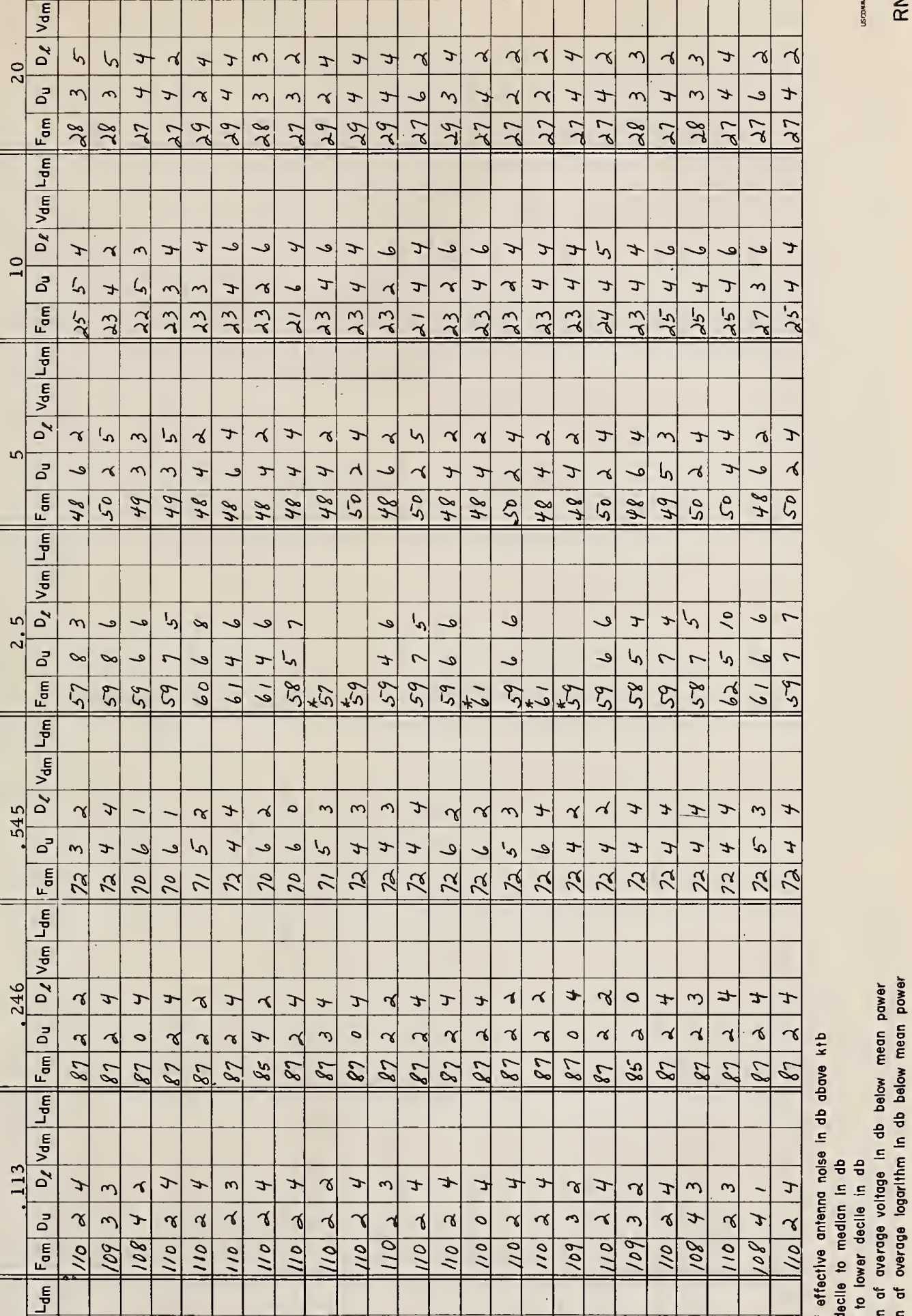

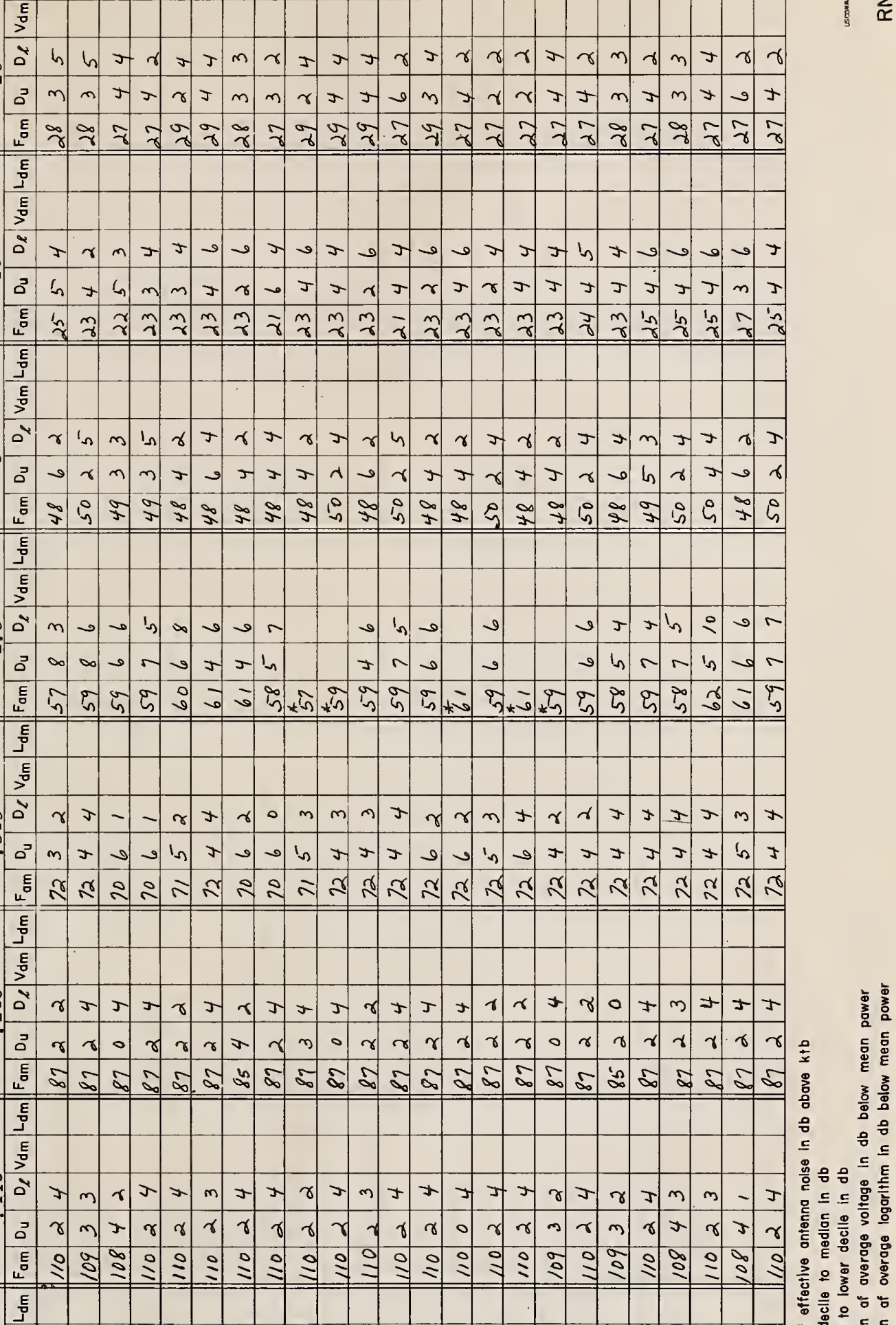

嵩 E्ㅁํ

음

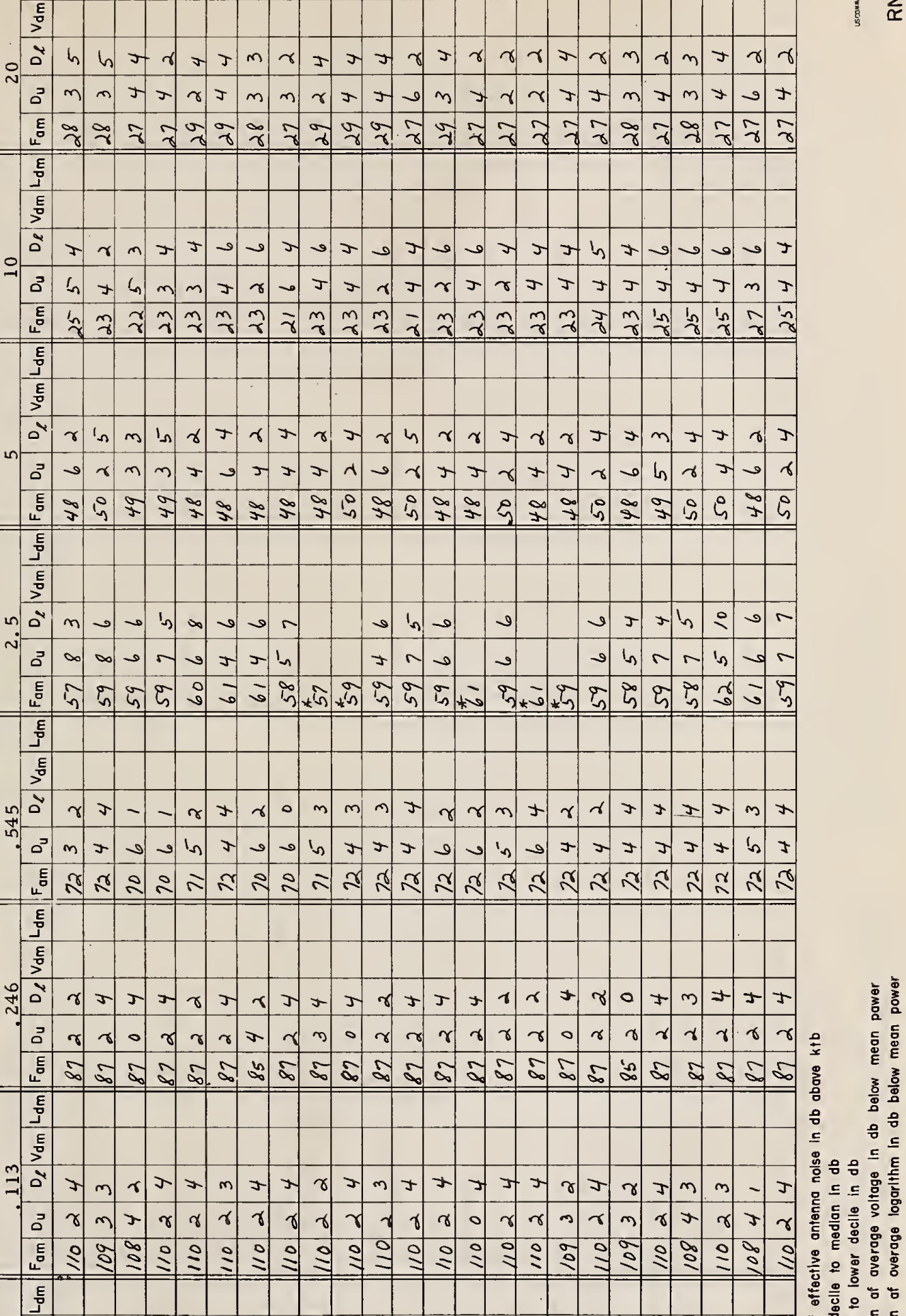

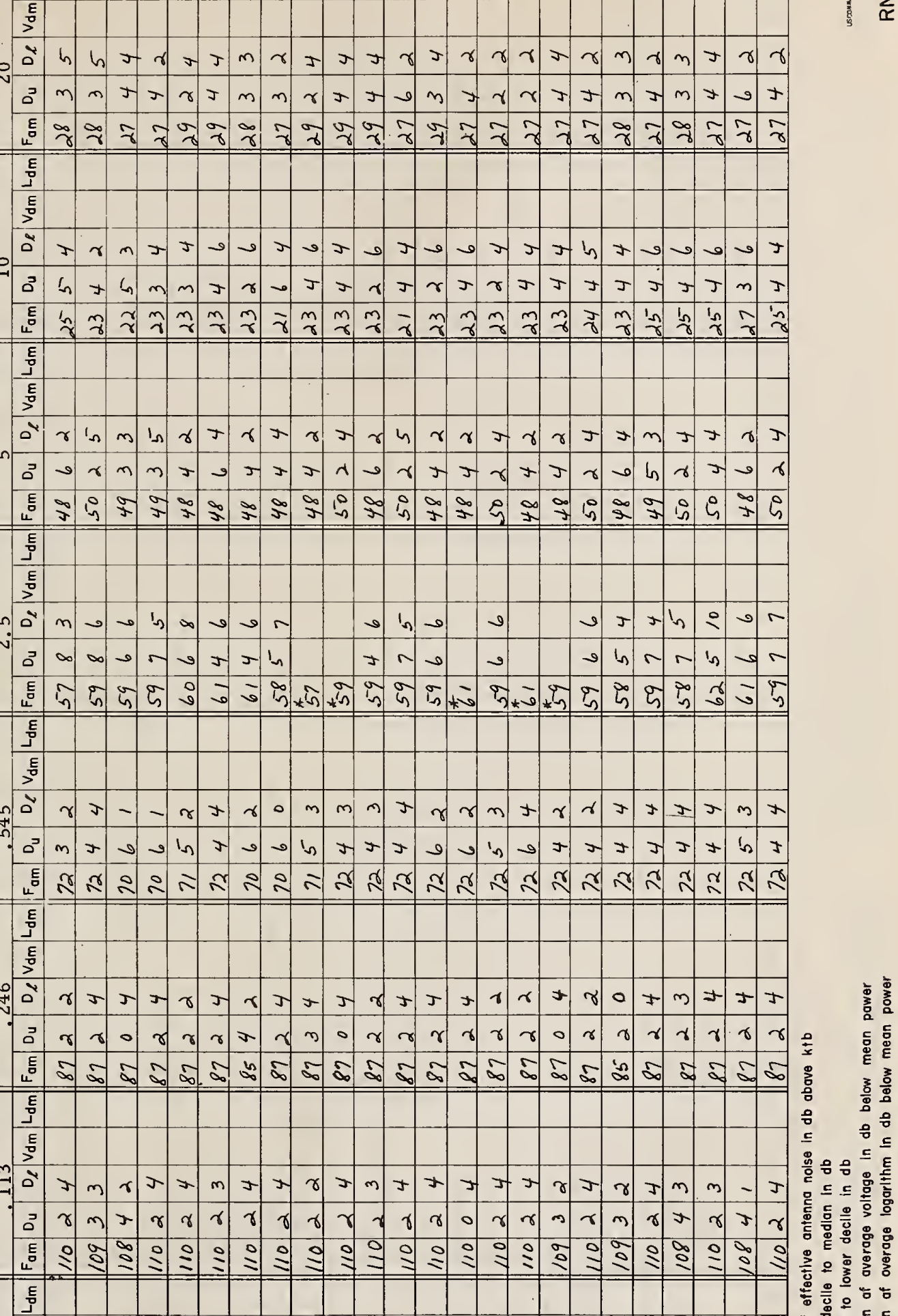

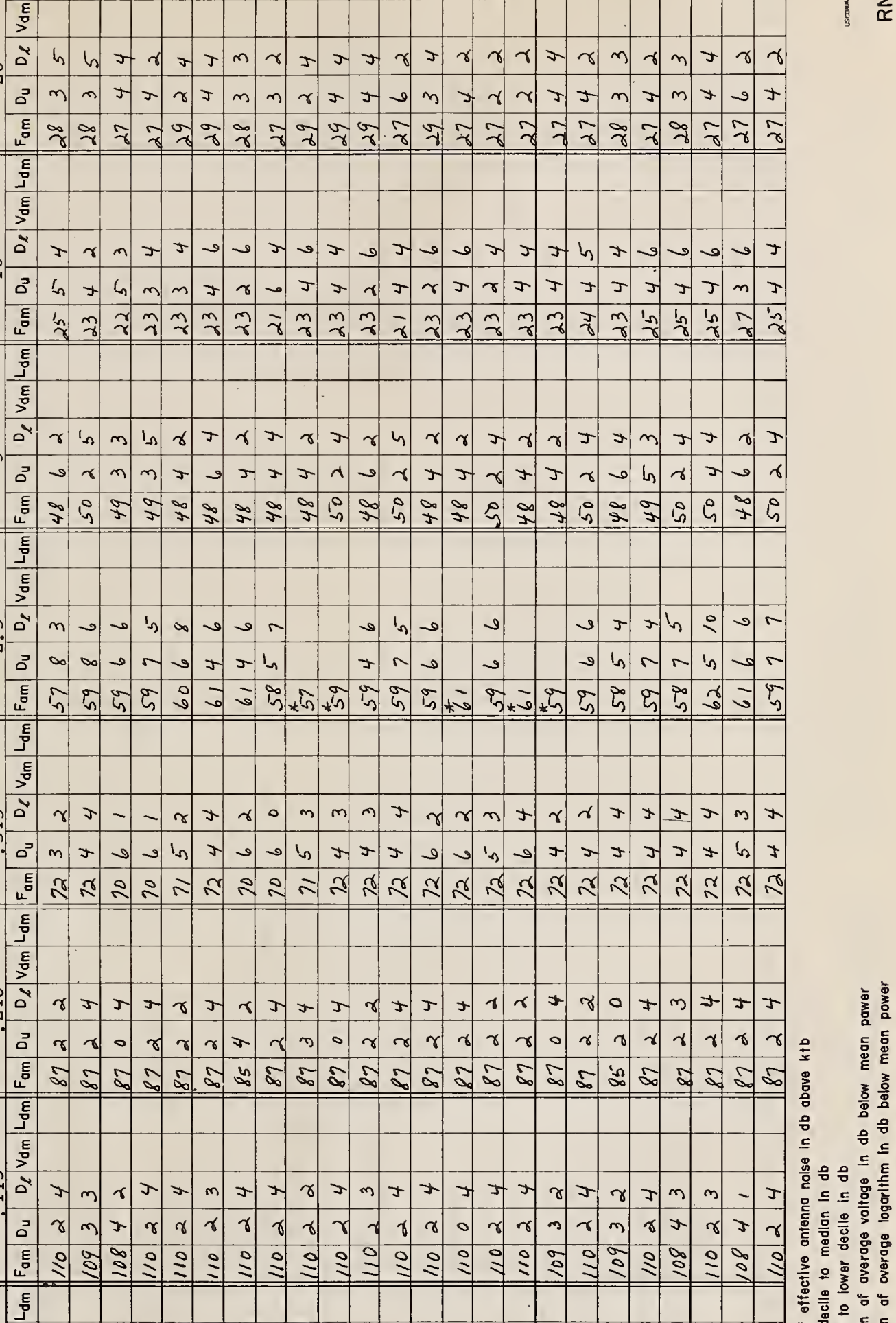
है

岁 E

㟧

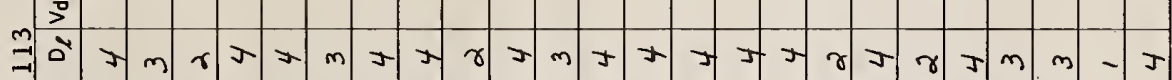

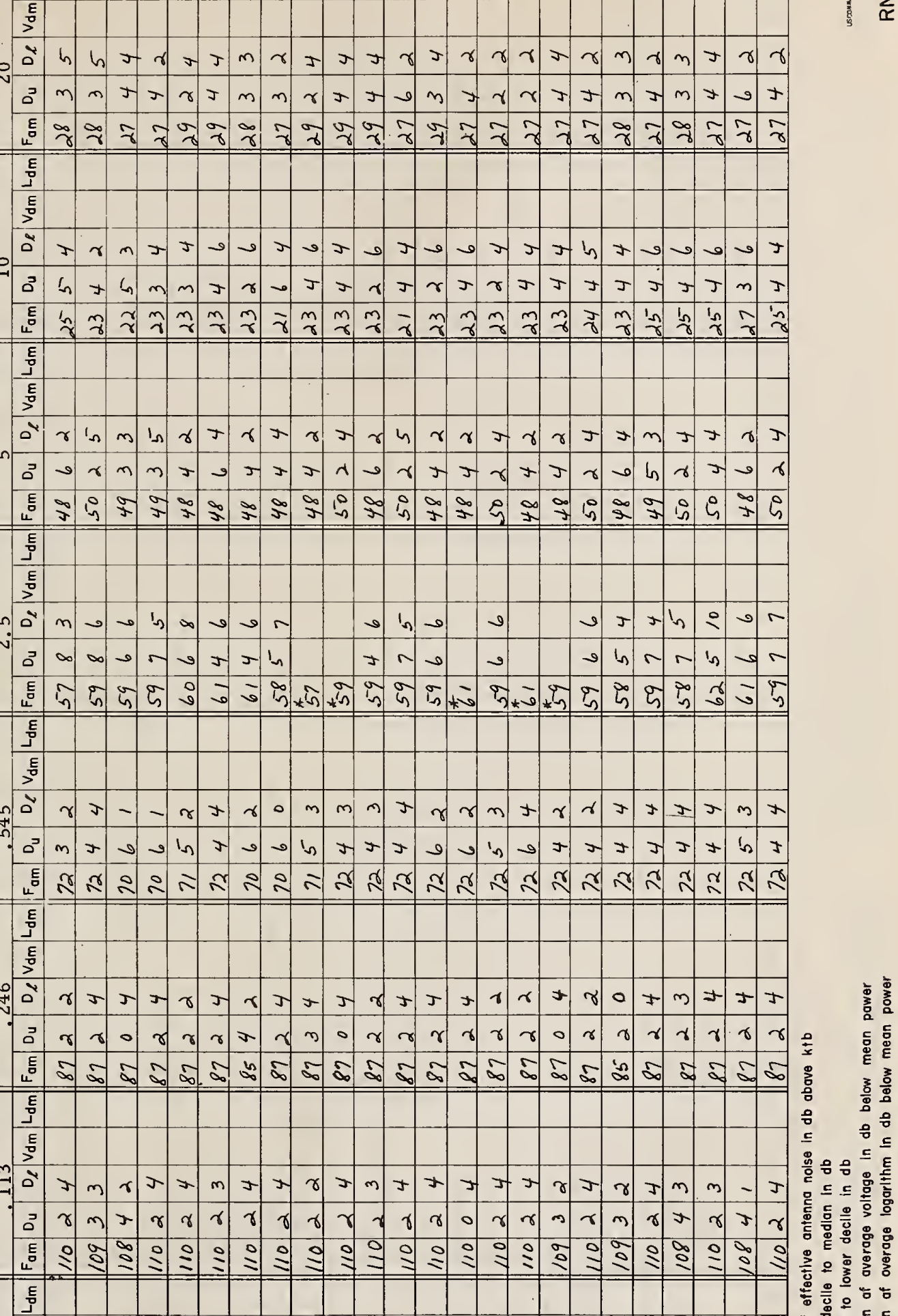

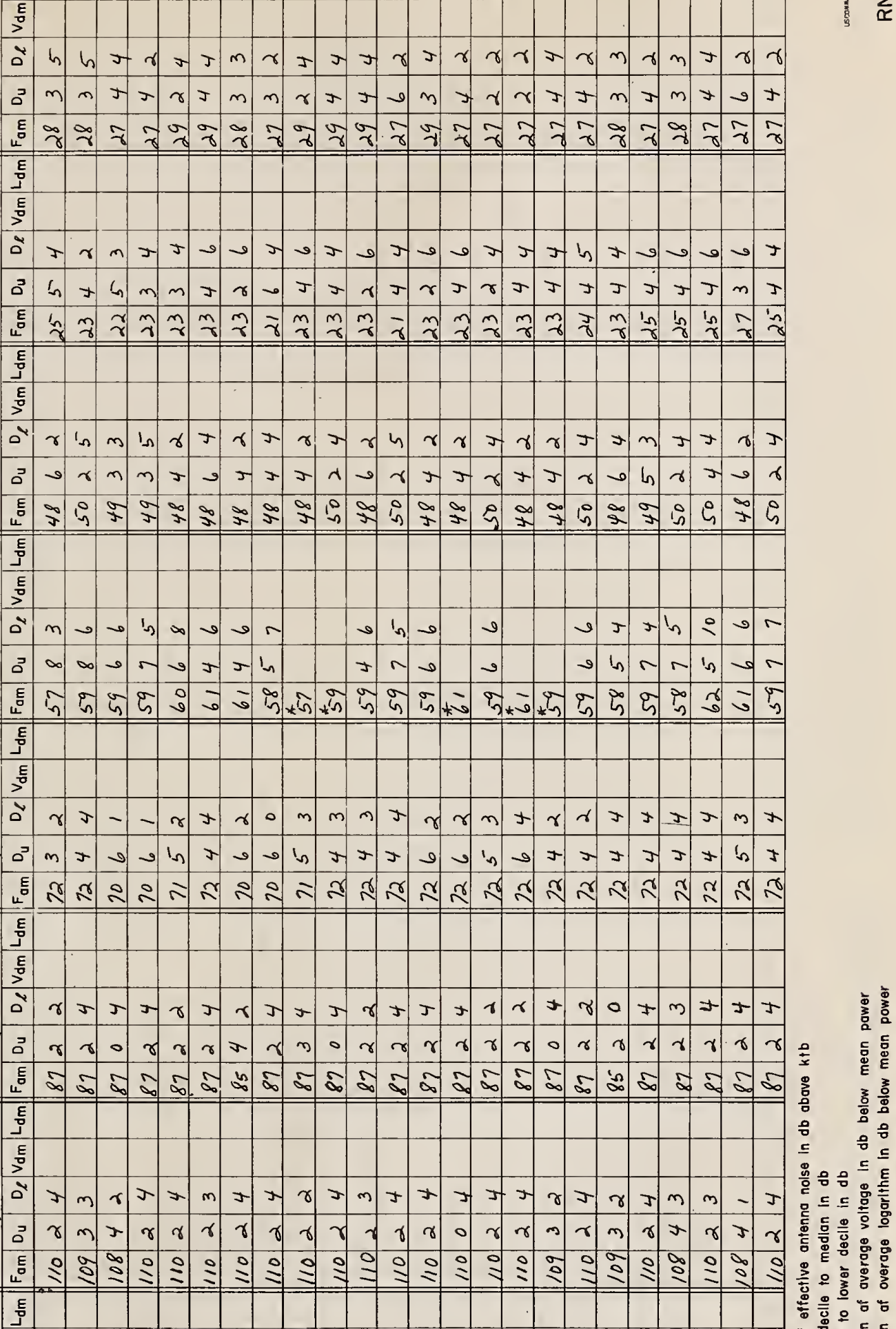

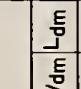

ลี $1-1$ 


$$
\text { E⿱ }
$$
แ

㟧 4

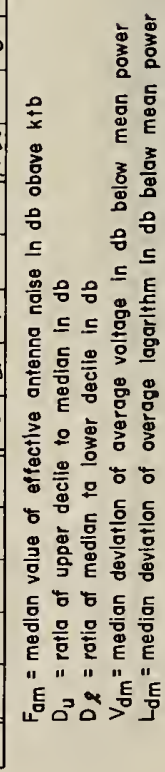


in

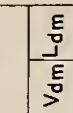

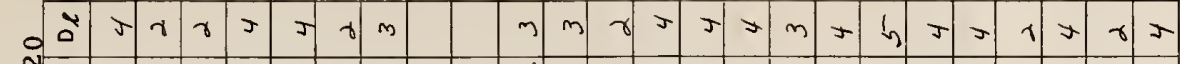

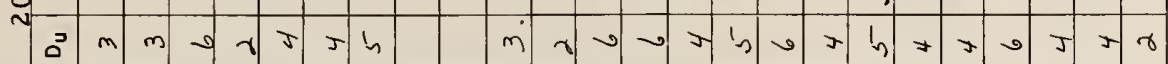

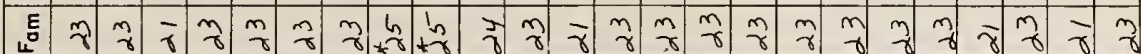

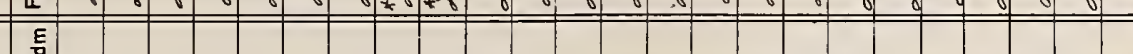

产

E

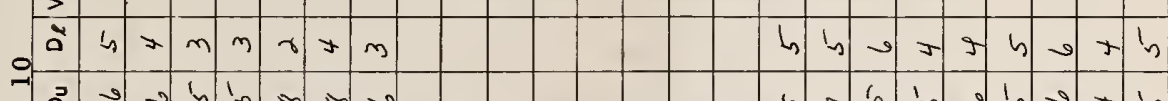

in

ஜ்

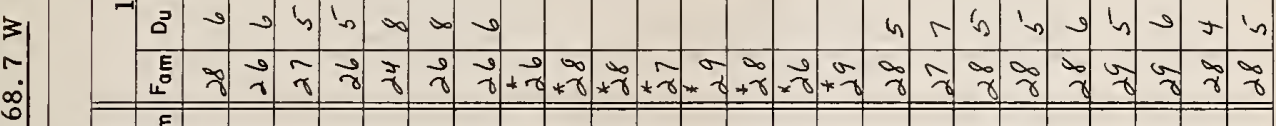

$0+\infty \rightarrow \infty \infty \infty$

$a+h \rightarrow \infty \rightarrow \infty$

$a \rightarrow \infty$ ar $\rightarrow$ a s

$?$

+ே ह

렴 E

$\ln 20 \rightarrow \infty \rightarrow \infty \rightarrow \infty$

כ)

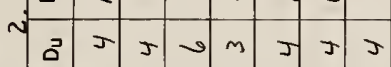

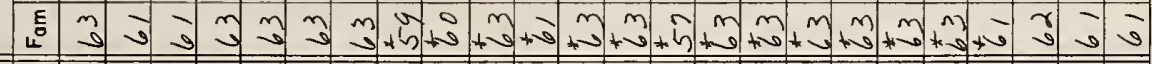

它 $\frac{E}{\partial}$

要

nू mar

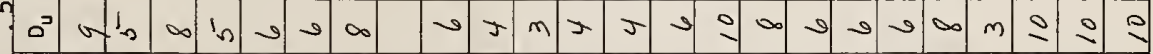
E)

峞

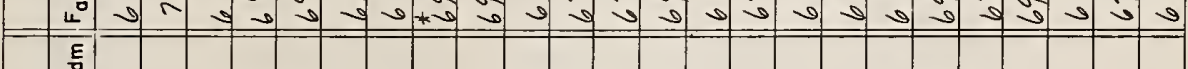

울

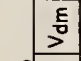

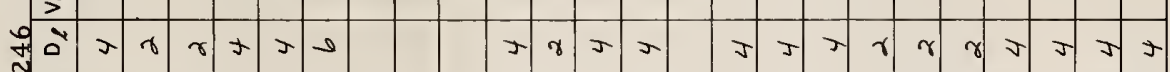

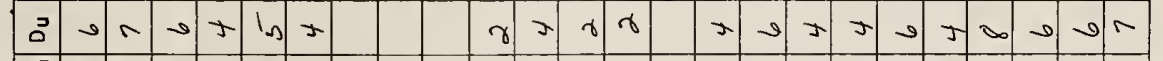

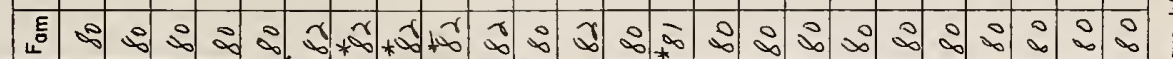

눙

$\frac{8}{3}$

告

岂

$m=\frac{1}{2} \rightarrow$

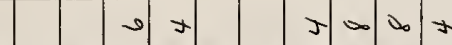

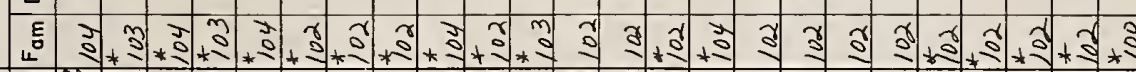

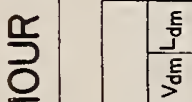

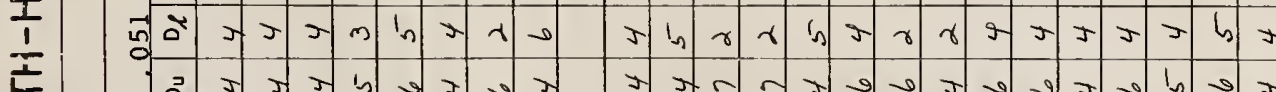

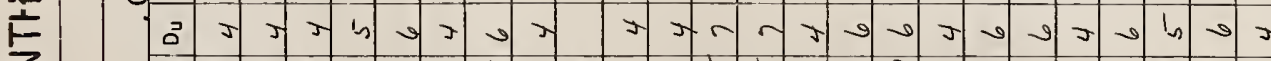

Z

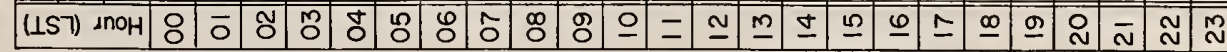




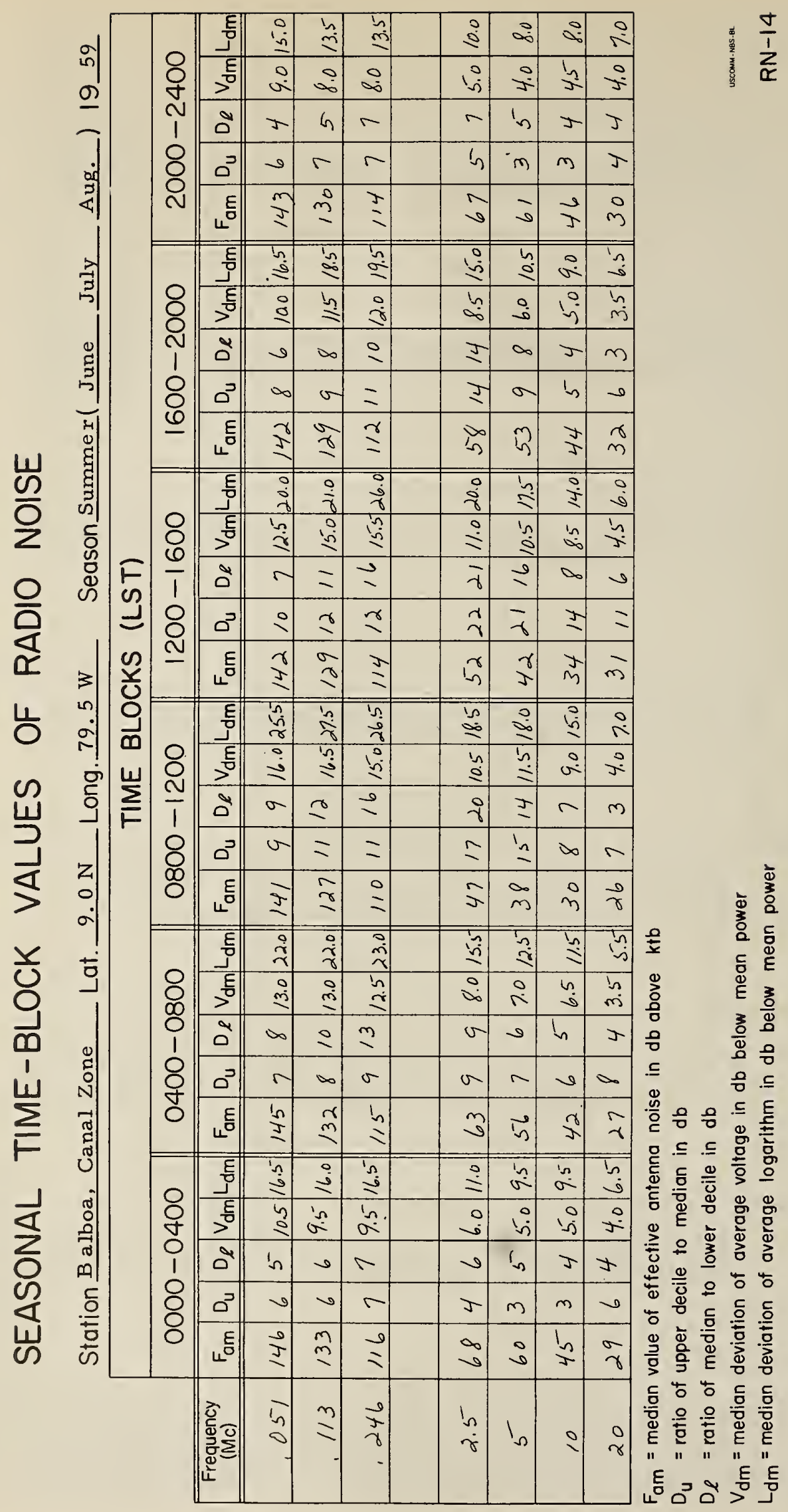




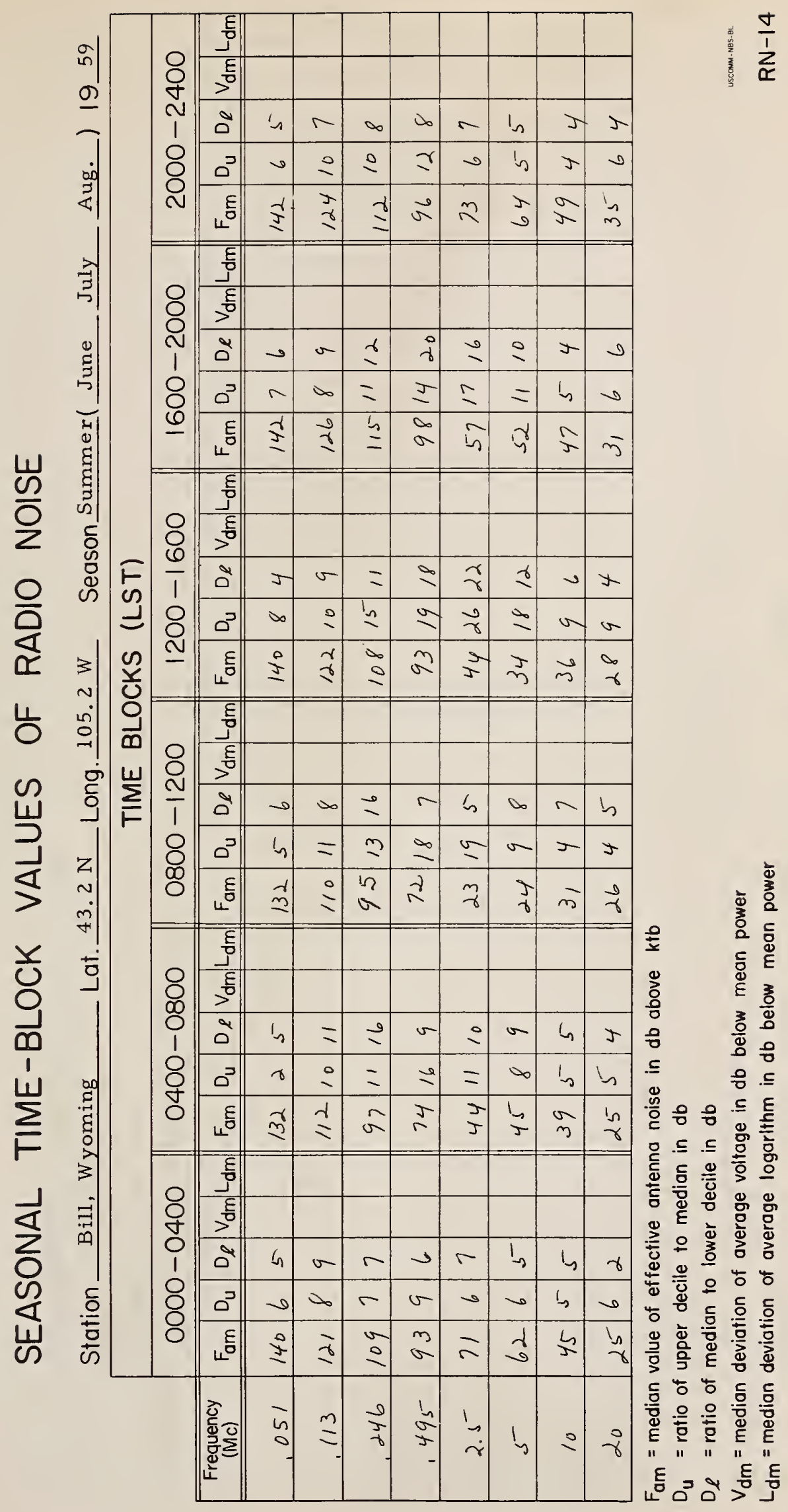




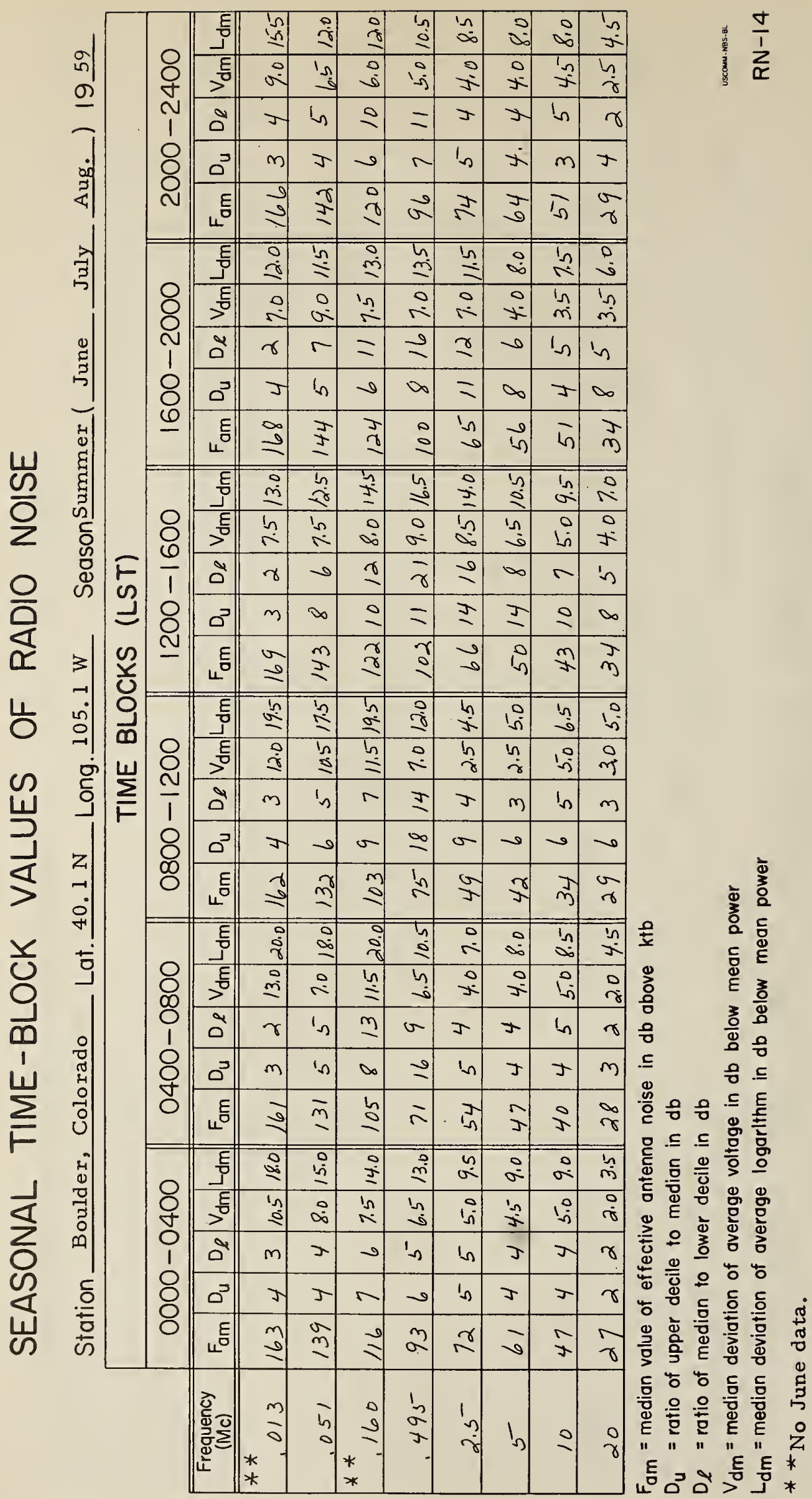




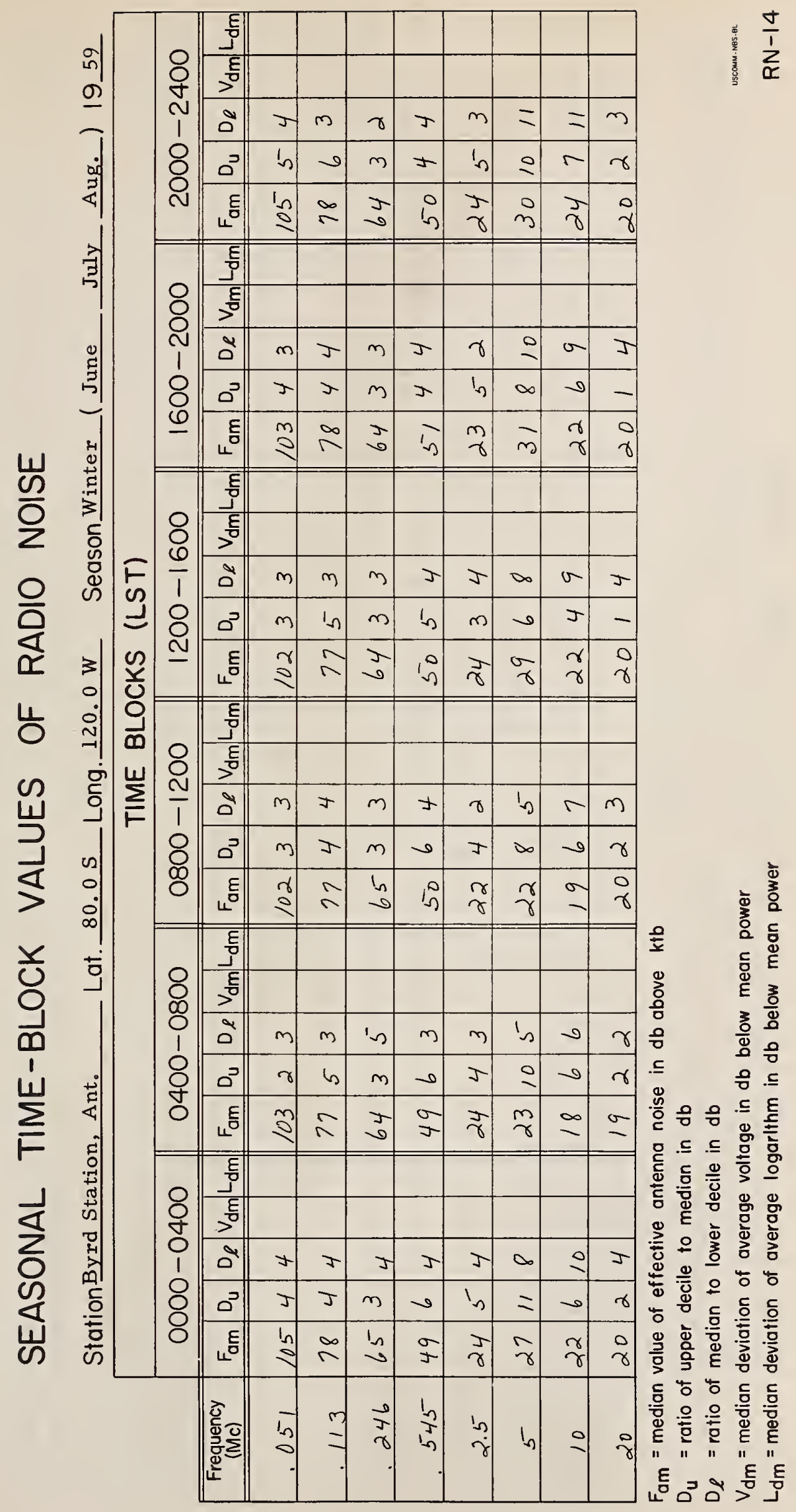




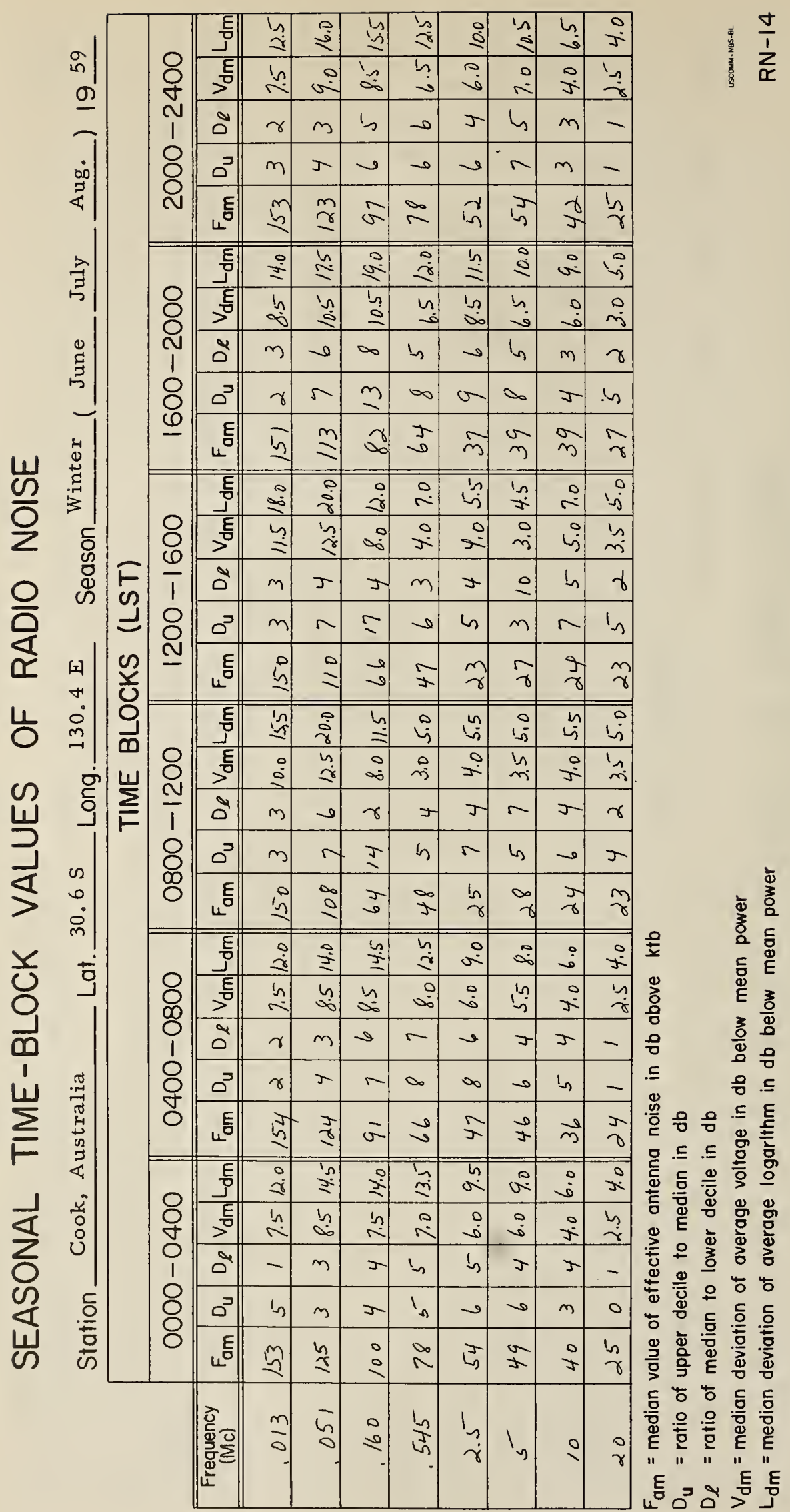




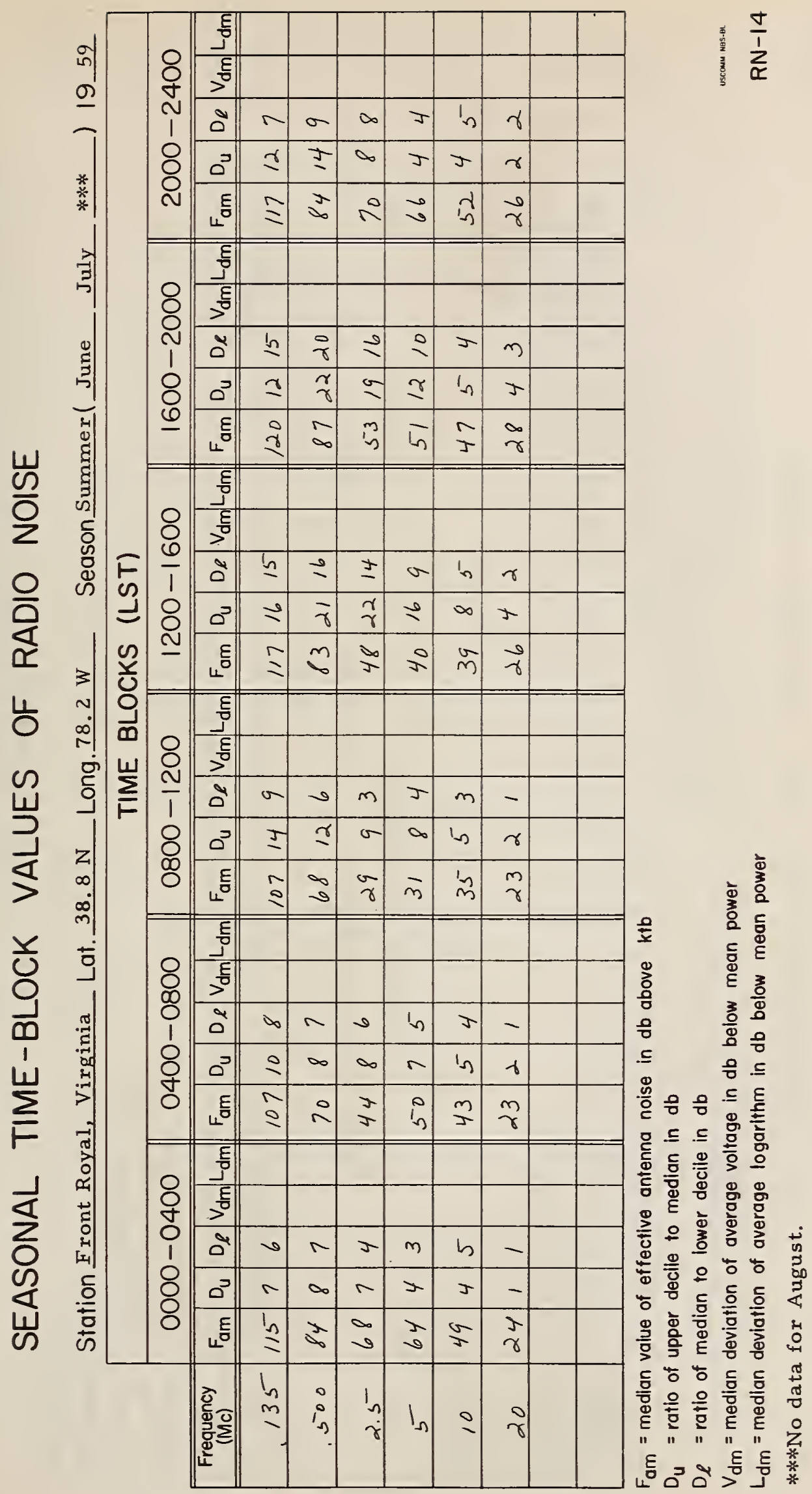




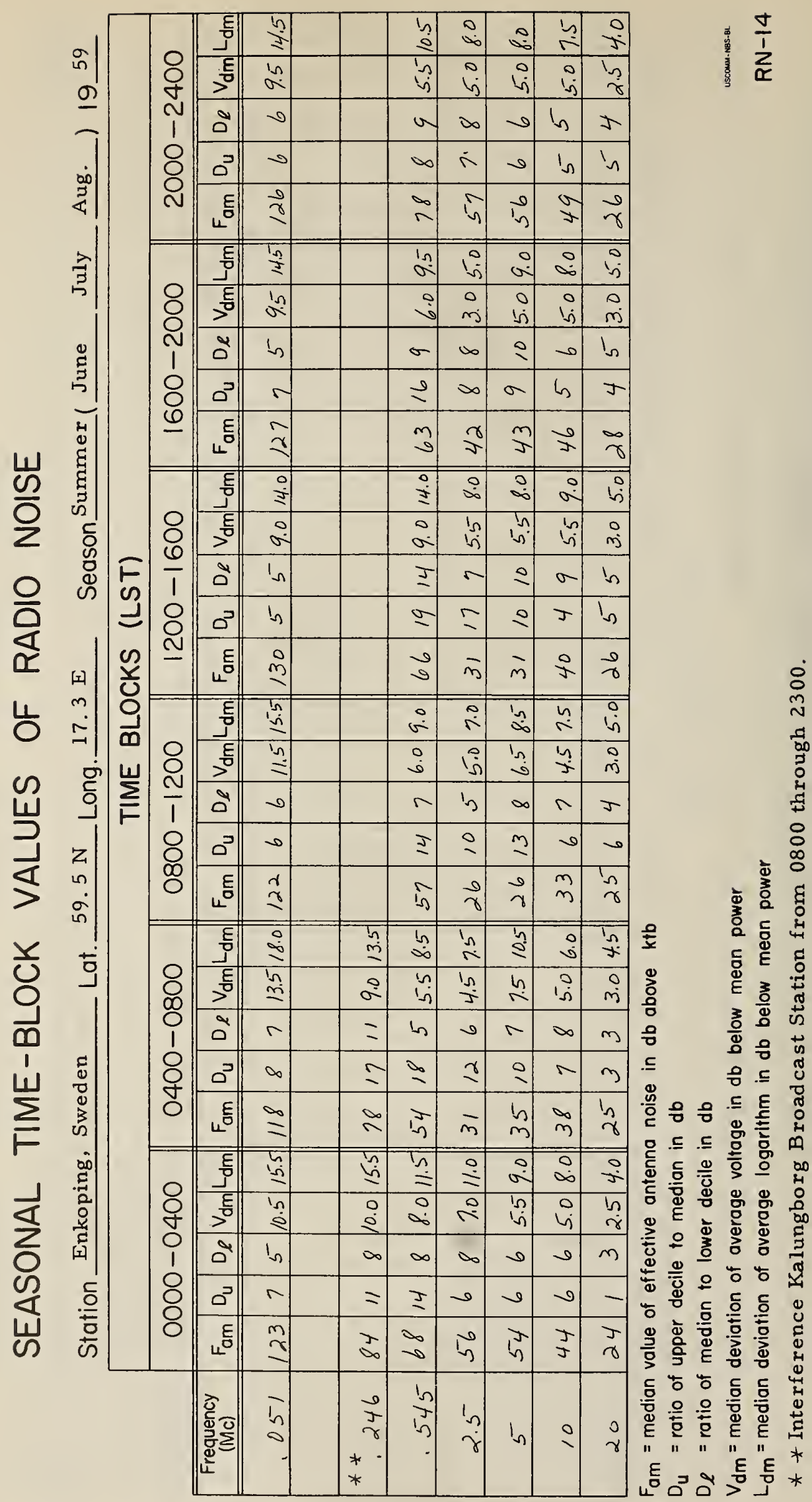




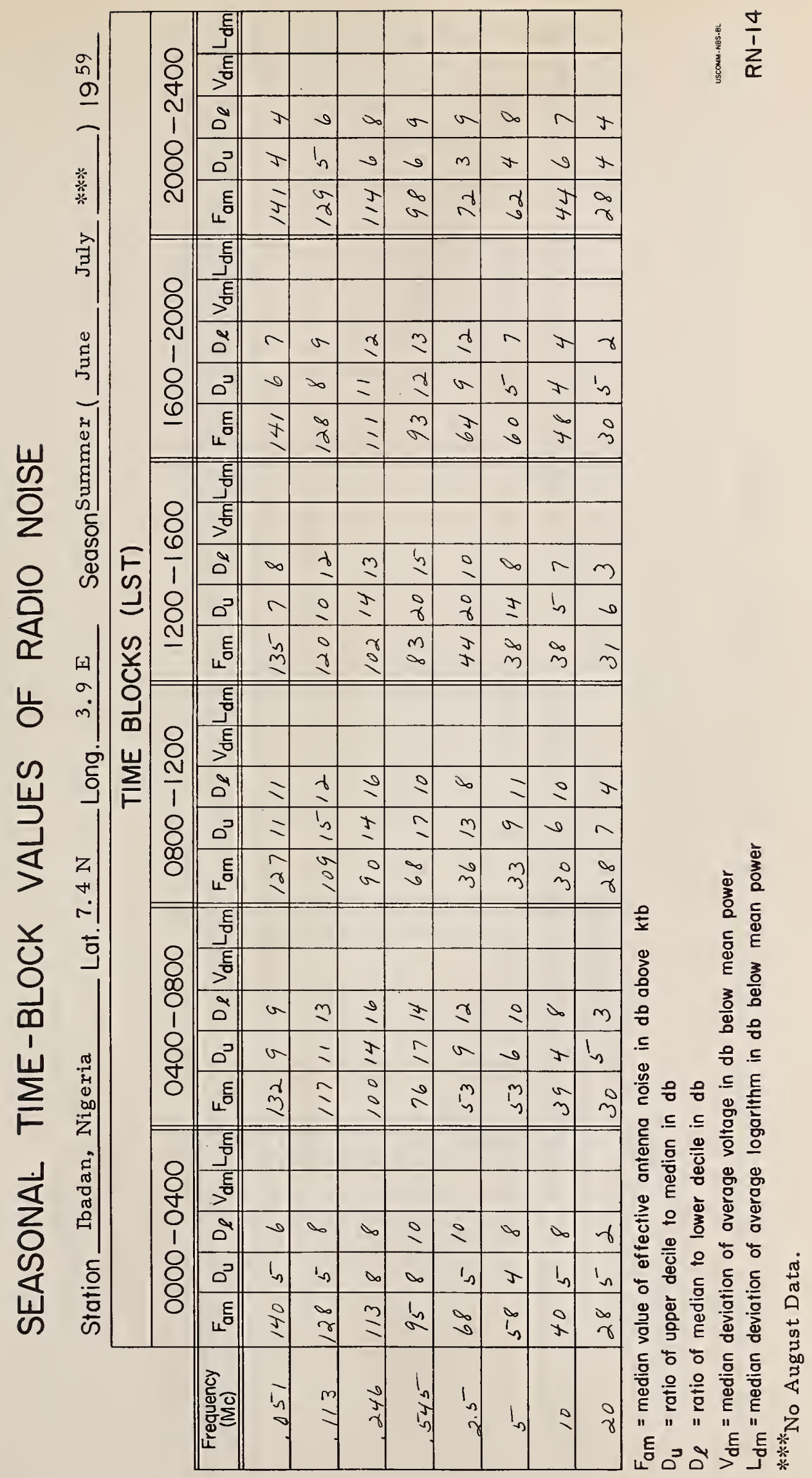




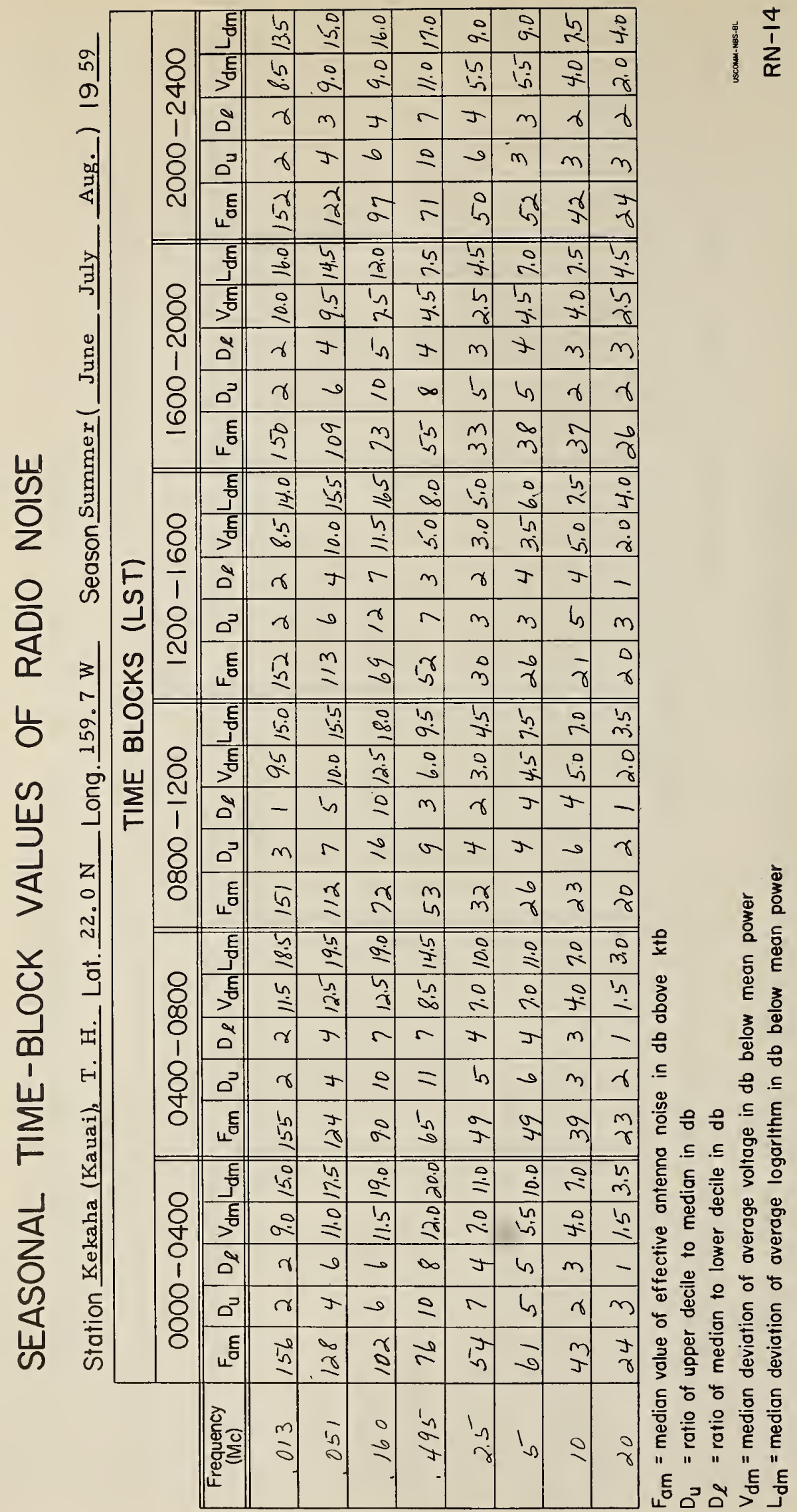




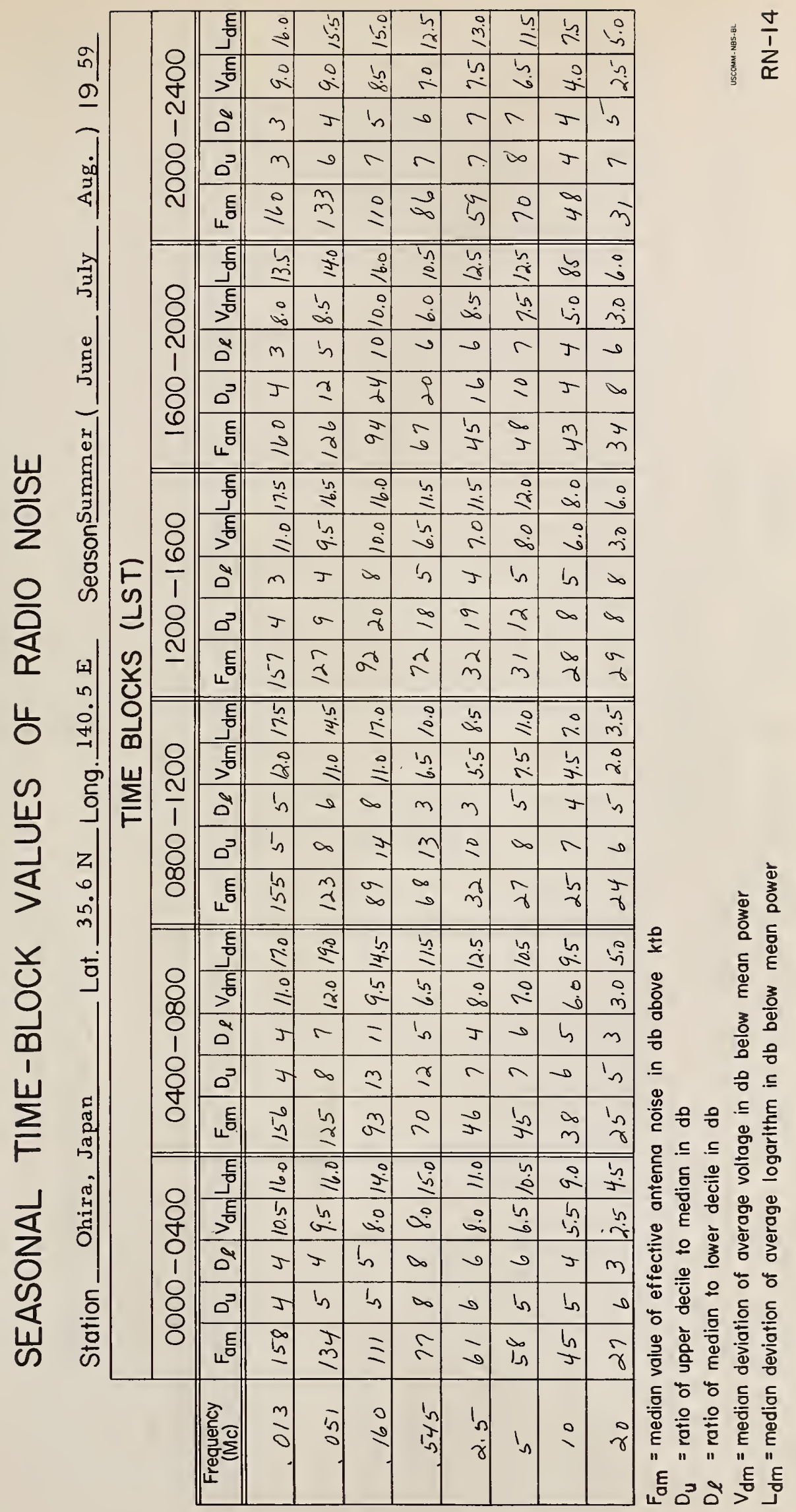




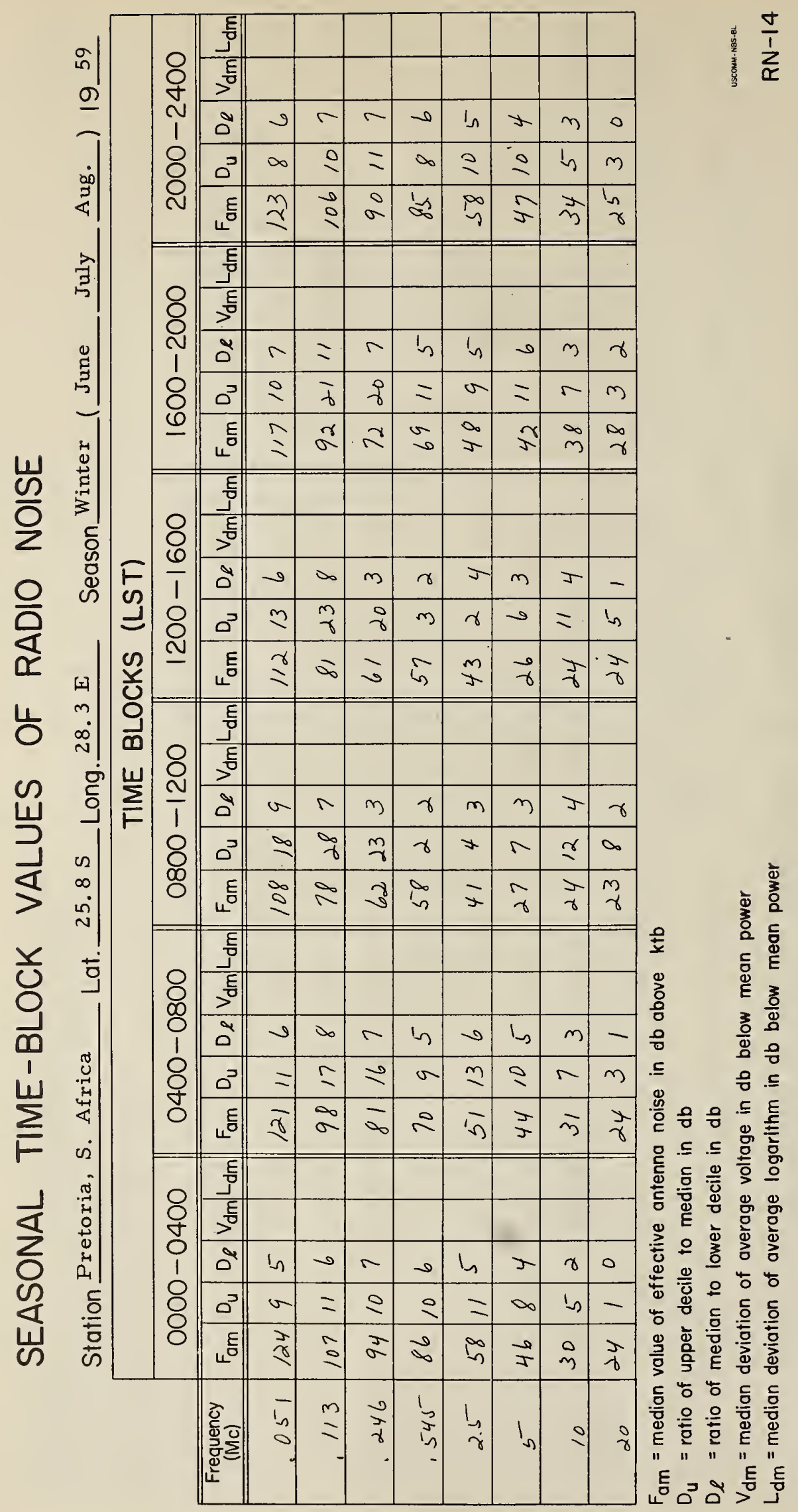




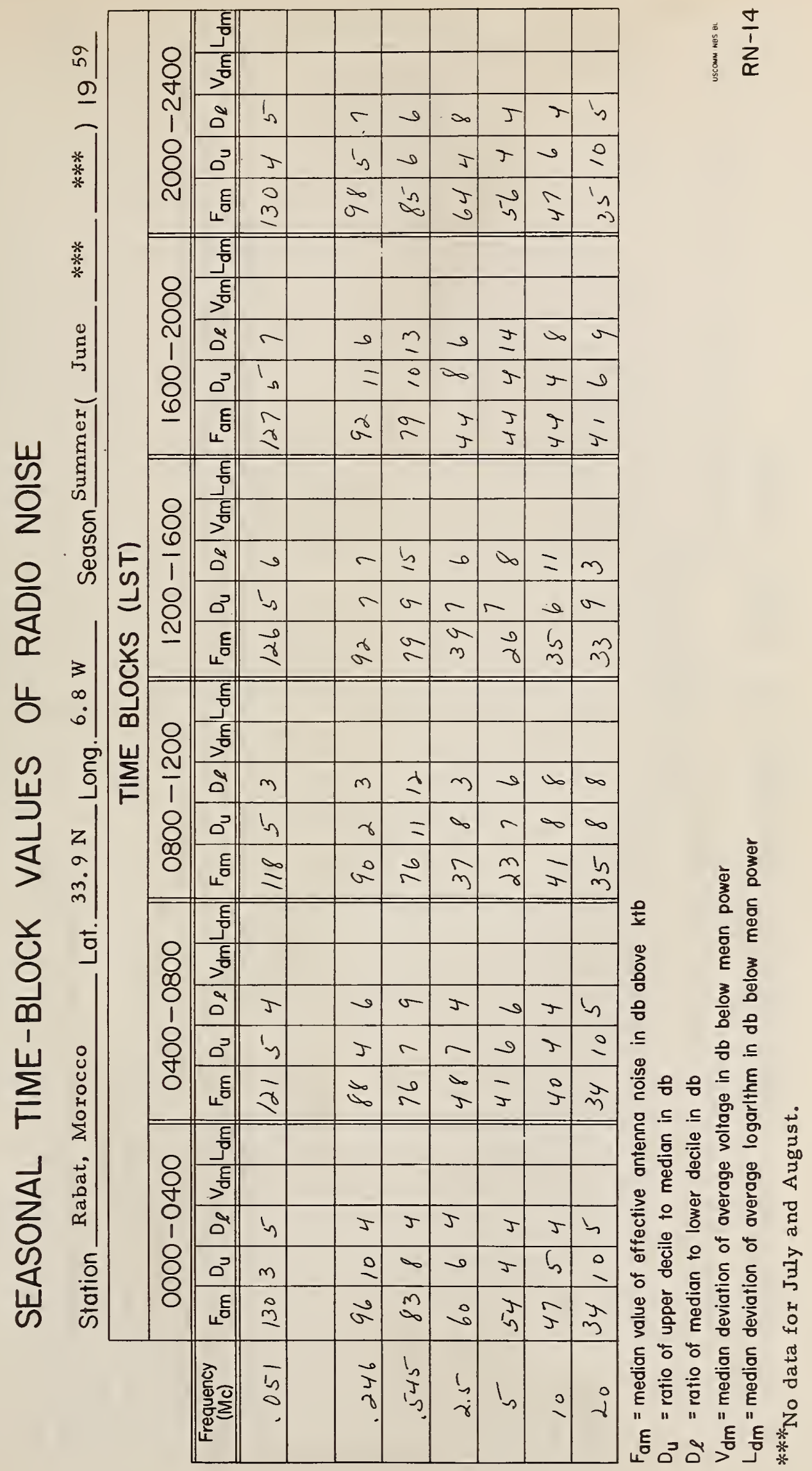




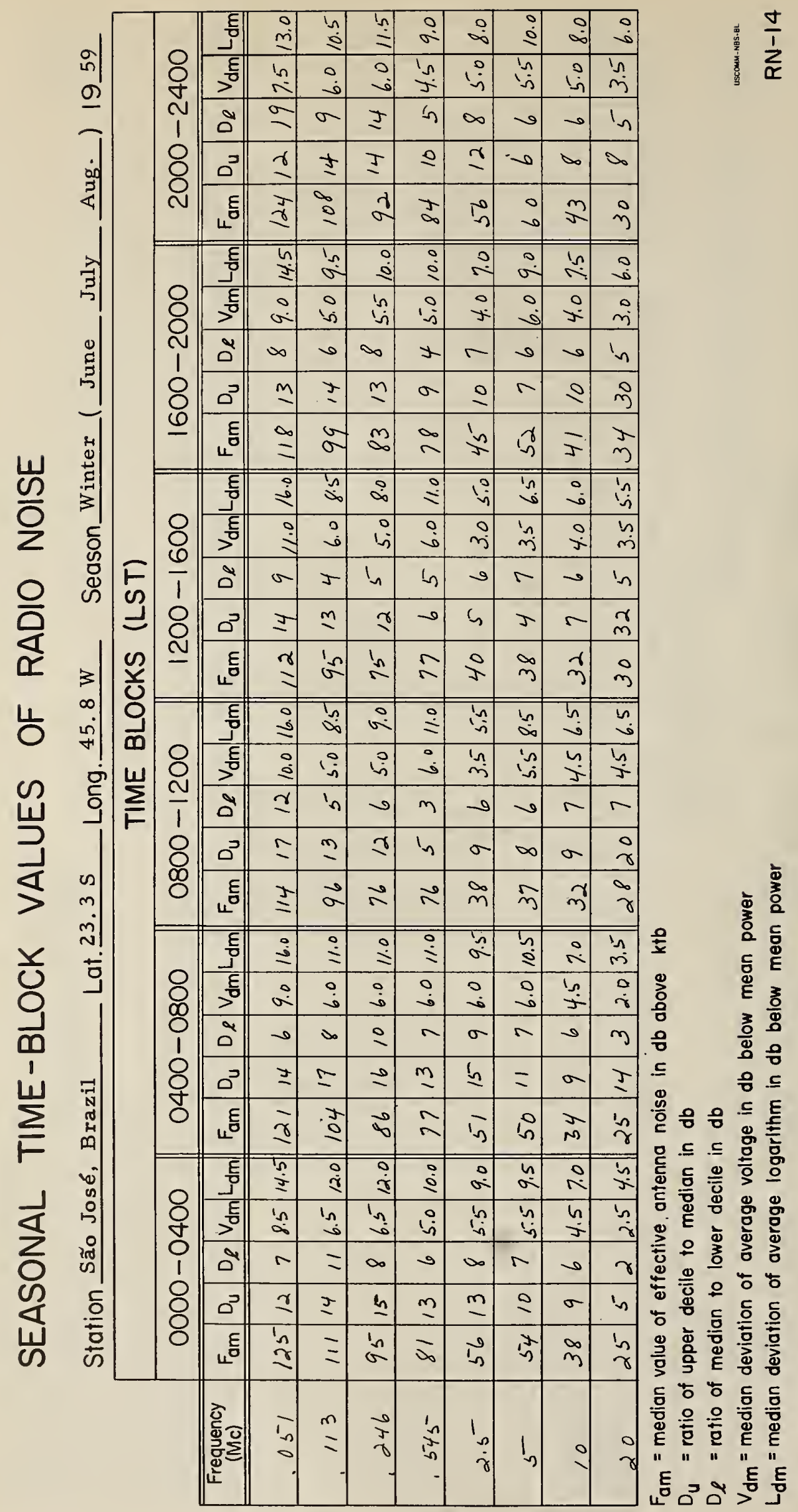




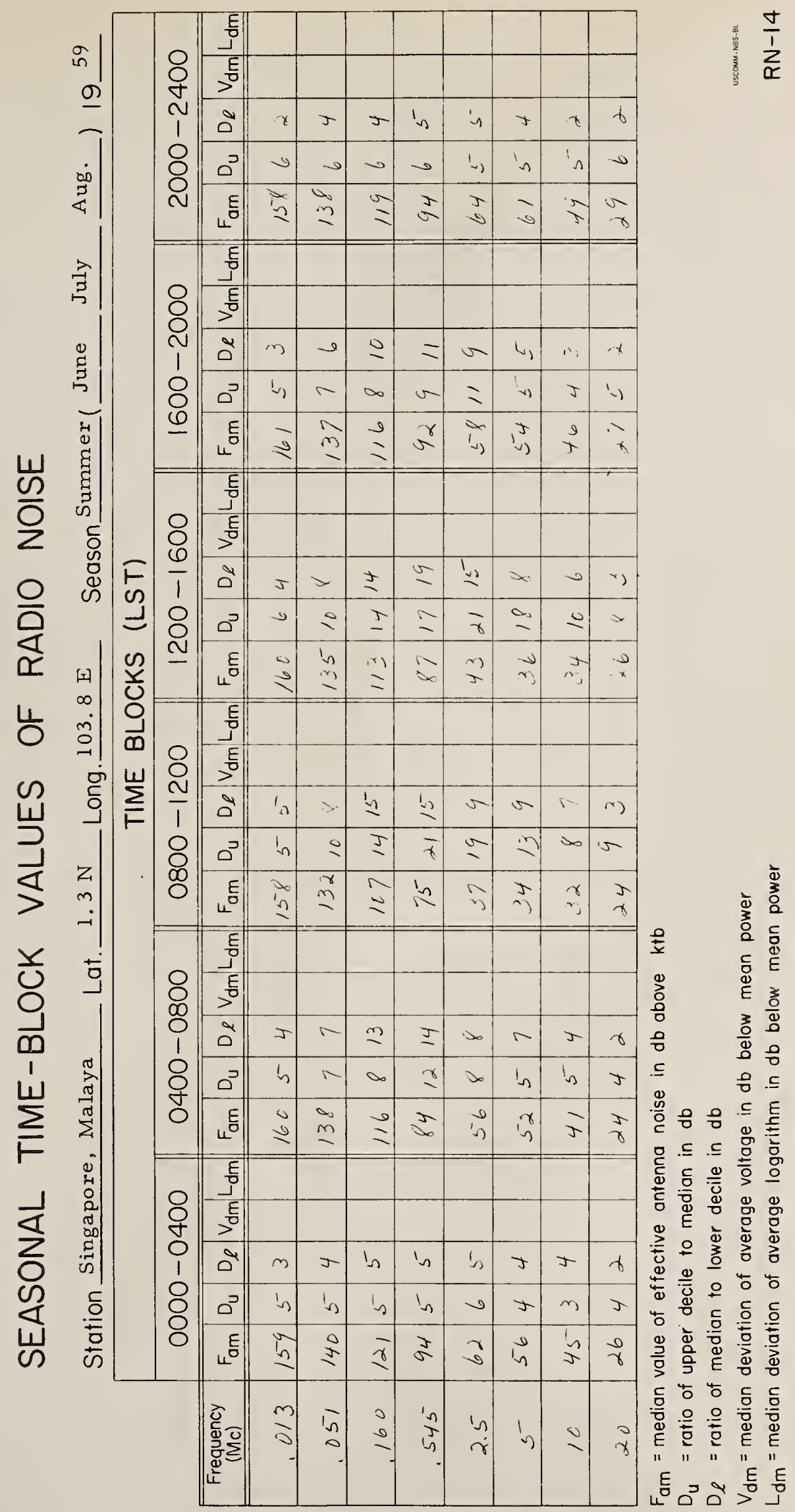




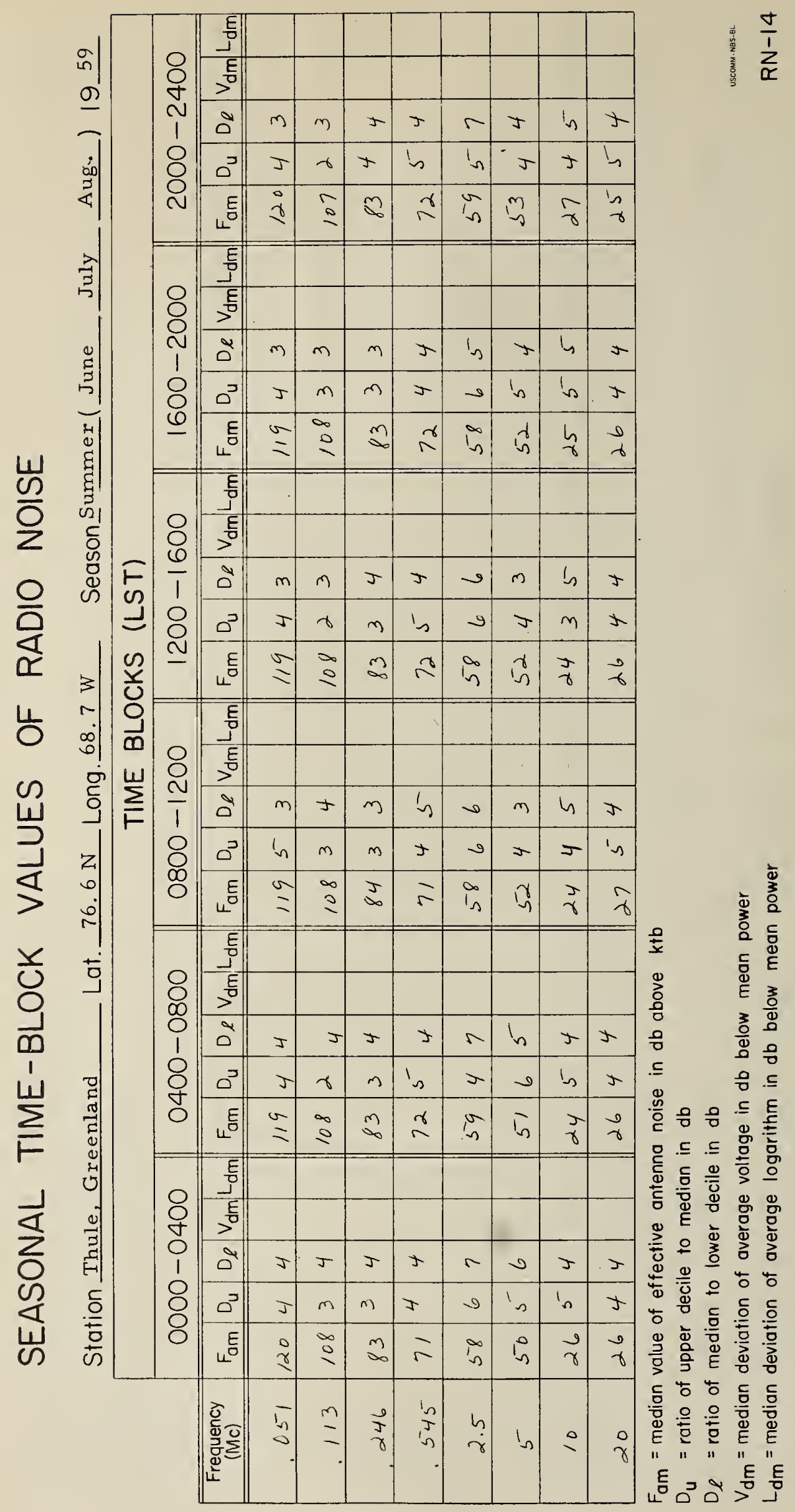




\section{THE NATIONAL BUREAU OF STANDARDS}

The scope of activities of the National Bureau of Standards at its inajor laboratories in Washington, D.C., and Bontler, Colorado, is suggested in the following listing of the divisions and sections engaged in technical work. In gencral, each section carrics out specialized rescarch. development, and engineering in the field indicated by its title. I brief description of the activities, and of the resultant publications, appears on the inside of the front cover.

\section{WASHINGTON, D.C.}

Elcctricity and Electronies. Resistance and Reactance. Electron Devices. Electrical Instruments. Magnetic Measurements. Dielectrics. Engineering Electronics. Electronic Instrumentation. Electrochemistry.

Optics and Metrology. Photonctry and Colorimetry. Photographic Technology. Length. Engincering Metrology.

Heat. Temperature Physies. Thermodynamics. Cryogenic Physies. Rhoology. Molecular Kinetics. Free Radicals Rescarch.

Atomic and Radiation Physics. Spectroscopy. Radiometry. Mass Spectrometry. Solid State Physics. Electron Physics. Atomic Physies. Neutron Physics. Radiation Theory. Radioactivity. X-rays. Iligh Encrgy Radiation. Vucleonic Instrumentation. Radiological Equipment.

Chemistry. Organic Coatings. Surface Chemistry. Organic Chemistry. Analytical Chemistry. Inorganic Chentistry. Electrorleposition. Molecular Structure and Properties of Gases. Physical Chemistry. Thermochemistry. Spectrochemistry. Pure Substances.

Mechanics. Sound. Mechanical Instruntents. Fluid Mechanics. Enginecring Mechanics. Mass and Scale. Capacity, Density, and Fluid Meters. Combustion Coutrols.

Organic and Fibrous Matcrials. Rubber. Textiles. Paper. Leather. Testing and Specifications. Polyiner Structure. Plastics. Dental Rescarch.

Metallurgy. Thermal Metallurgy. Chemical Metallurgy. Mechanical Metallurgy. Corrosion. Mctal Physics.

Mineral Products. Engineering Ceramics. Glass. Refractories. Enameled Metals. Constitution and Microstructure.

Building Technology. Structural Engineering. Fire Protection. Air Conditioning, Heating, and Refrigcration. Floor, Roof, and $W$ all Coverings, Cortes and Safety Standards. Heat Transfer. Concreting Materials.

Applied Mathematics. Numerical Analysis. Computation. Statistical Engineering. Mathematical Physics.

Data Processing Systems. SEAC Engineering Group. Components and Techniques. Digital Circuitry. Digital Systenıs. Analog Systems. Application Engineering.

- Office of Basic Instrumentation.

- Office of Weights and Measures.

\section{BOULDER, COLORADO}

Cryogenic Engincering. Cryogenic Equipment. Cryogenic Processes. Properties of Materials. Gas Liquefaction.

Radio Propagation Physics. Lpjer Atmosphere Research. Ionospheric Research. Regular Propagation Services. Sun-Earth Relationships. VIIF Research. Radio Warning Services. Airglow and Aurora. Radio Astronomy and Arctic Propagation.

Radio Propagation Engincering. Data Reduction Instrumentation. Modulation Rescarch. Radio Noise. Tropospheric Measurements. Tropospheric Analysis. Propagation Obstacles Engineering. Radio-Meteorology. Lower Atmosphere Pliysics.

Radio Standards. High Frequency Electrical Standards. Radio Broarlcast Service. Iligh Frequency Inpedance Standards. Electronic Calibration Center. Microwave Physirs. Microwave Circuit Standards.

Radio Communication and Systems. Low Frequency and Very Low Frequency Rescarch. IIigl lirequency and Very High Frequency Research. Ultra High Frequency and Super Il igl Frequeney Research. Moduktion Research. Antenna Rescarch. Vavigation Systeıns. Systems Analysis. Fiehl Operations. 


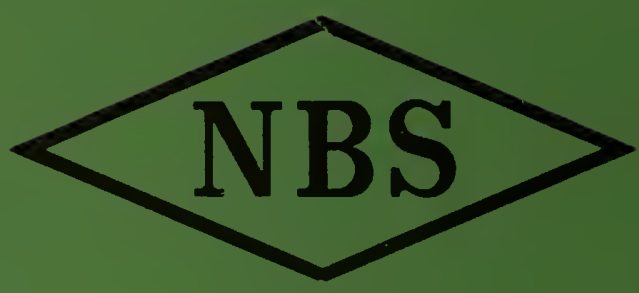

